Bangladesh J. Plant Taxon. 28(1): 27-60, 2021 (June)

(C) 2021 Bangladesh Association of Plant Taxonomists

https://doi.org/10.3329/bjpt.v28i1.54207

\title{
FLORISTIC COMPOSITION OF JAHANGIRNAGAR UNIVERSITY CAMPUS - A SEMI-NATURAL AREA OF BANGLADESH
}

\author{
Saleh Ahammad Khan, Sharmin Sultana, Gazi Mosharof Hossain, \\ Shayla Sharmin Shetu* and Md. Abdur Rahim \\ Plant Systematics and Biodiversity Laboratory, Department of Botany, \\ Jahangirnagar University, Savar, Dhaka, Bangladesh
}

Keywords: Flora; Angiosperms; Semi-natural; Jahangirnagar; Bangladesh.

\begin{abstract}
This study represents comprehensive taxonomic information on current floristic composition in the semi-natural campus area of Jahangirnagar University. This study reveals that this campus area harbours a total of 917 species belonging to 574 genera and 145 families of vascular plants. $70.34 \%$ of these species are wild and the rest are cultivated/planted. $63.79 \%$ of the species are native and $36.21 \%$ are exotic to Bangladesh. Pteridophytes and Gymnosperms are represented by 22 and 12 species, respectively, whereas, Angiosperms by 883 species. Magnoliopsida (dicotyledons) and Liliopsida (monocotyledons) are composed of 618 and 265 species, respectively. The family Poaceae with 89 species is the largest, which is followed by Fabaceae with 44 species and Asteraceae with 40 species. Most of the species are herbs (56.16\%) and growing in diverse habitats. All species are economically or ecologically important, but mostly as medicinal, ornamental, fodder and forage, fruit, vegetable, timber and fuel wood. The study area houses 15 plant species, previously listed as threatened to Bangladesh. Thus, the study area is floristically rich in respect to its number of plant species belonging to different groups and its size. This area might serve as a campus-based excellent center of biodiversity conservation by strengthening the existing management and adopting necessary policies and strategies.
\end{abstract}

\section{Introduction}

Urbanization is one of the main drivers of ecological, environmental and socio-economical change worldwide (Freitas et al., 2020). It is a central component of land-transformation processes and fundamental changes in land use and landscape pattern around the globe, especially in the developing countries. The consequences of urbanization result in drastic changes to the biodiversity and composition of plant communities and even lead to species extinction (McKinney, 2006; Freitas et al., 2020).

A semi-urban or peri-urban area is a transitional component of urbanization, which is usually resulted through the dynamic and fast transformation of rural land or natural tract into urban land (Meeus and Gulinck, 2008). In many cases, the semi-urban areas are in the vicinity and under influence of urban cores (Cazaux et al., 2007). If a semi-urban area is semi-natural, it may form its own landscapes and may have some intact ecological and biodiversity assemblages that have been substantially modified in their composition, balance, strength or function, largely by human activities.

There are immense benefits of conserving the vegetation of urban or semi-urban areas, especially in scientific, social, economic and environment issues (Hunter, 2007). Open space within such areas has beneficial effects on its microclimate, hydrology, biodiversity and ecological

*Corresponding author. Email: shaylaju819@gmail.com 
processes (Goddard et al., 2009). Maintaining biodiversity and natural environments in urban or semi-urban areas, is one of the biggest conservation challenges today (Kowarik, 2011). However, the first key step to achieve this goal is to compile the updated lists of taxa of these areas (Schaminée et al., 2011; Sharrock, 2012).

The campus of Jahangirnagar University (JU) is an eye-pleasing semi-natural semi-urban area in close proximity to Savar upazila town and Dhaka metropolitan city (Fig. 1). Once, this campus area was an integrated part of the Madhupur Tract harbouring the major part of the deciduous 'Sal' (Shorea robusta) forest of this country. The original deciduous forest vegetation of this area has been almost replaced by a mixed type of secondary vegetation and plantation forests.

Despite not being completely natural, the habitats and ecosystems of this area has high value in term of its rich biodiversity in its diverse ecological habitats, viz. open scrub jungles, grasslands, fallow lands, wetlands, gardens, agricultural lands, and woodlands including the scattered plantation forests and remnants of natural deciduous forest, and the important services it provides. Therefore, conducting studies on the biodiversity of this semi-natural area is necessary to know the extent and status of this natural resource and the trend of its changes under various anthropogenic pressures including urbanization, and to estimate the scope of conservation there.

In this country, the floristic explorations conducted so far are centered on the forested areas (Khan and Huq, 2001; Uddin and Hassan, 2004; Uddin and Hassan, 2010; Rahman et al., 2015; Islam et al., 2016; Rahman, 2017; Rahman et al., 2017; Haque et al., 2018). Studies on the flora of urban or semi-urban areas has long been neglected by the taxonomists, because the ecosystems of these areas have been regarded as highly disturbed and supporting relatively common species only. However, some taxonomic surveys have been completed on or covering some (semi-) urban areas (Alam et al., 2006; Sultana, 2012; Rahman et al., 2013; Tabassum, 2015; Shetu et al., 2018; Khanam et al., 2020; Roy and Khan, 2020). Studies on the flora of few campus areas have also been conducted (Momen et al., 2006; Uddin and Hassan, 2016). But so far no attention has been given to conduct a thorough taxonomic inventory on the flora of JU campus, except the listing of some vascular plant species as a part of the ecological studies (Hossain et al., 1995; Farhana et al., 1997). Therefore, the scope of detail taxonomic study on the flora and assessment of plant diversity of this highly diverse campus area, especially in respect to conservation and management aspects, is still remaining. This study was conducted to complete a comprehensive taxonomic inventory on the vascular flora of JU campus area with highlighting the existing conservation and management aspects.

\section{Materials and Methods}

Jahangirnagar University (JU) campus, composed of 282.29 hectares of land, is situated in between $23.8671^{\circ}-23.8977^{\circ} \mathrm{E}$ and $90.2588^{\circ}-90.2731^{\circ} \mathrm{N}$, in Savar upazila of Dhaka district, Bangladesh, and $32 \mathrm{~km}$ north-west from the Dhaka city along the Dhaka-Aricha highway (Fig. 1). The topography of this area is slightly undulating and composed of red lateritic soil. In the study area, monthly average temperature varies from $30^{\circ}-41^{\circ} \mathrm{C}, 29^{\circ}-32^{\circ} \mathrm{C}$ and $19^{\circ}-25^{\circ} \mathrm{C}$, rainfall ranges from 12.1-154.2 mm, 106.42-350.8 $\mathrm{mm}$ and 0-19.5 mm and humidity differs from 42-82\%, 74$98 \%$ and $38-67 \%$ in summer, rainy and winter seasons, respectively according to the records of last one decade (www.worldweatheronline.com). The trend of monthly total rainfall in this area is decreasing in all seasons, whereas that of monthly mean temperature is increasing in summer but slightly decreasing in rainy and winter seasons conforming to the records of last three decades (http://datalibrary.bmd.gov.bd/maproom/Climatology/Climate). 


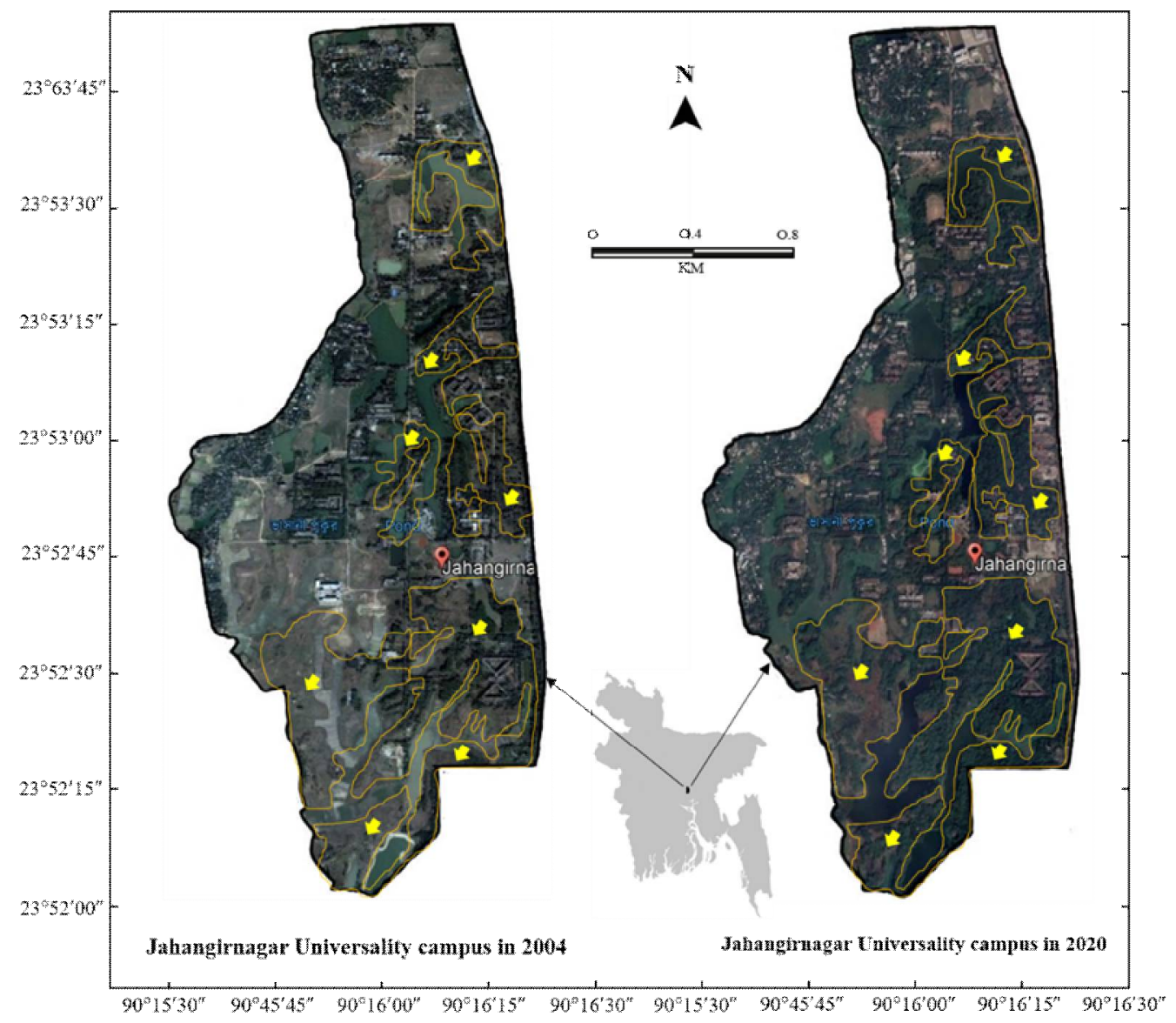

Fig. 1. Images on the study area in the years of 2004 and 2020 showing the change in vegetation cover of major (semi-) natural areas (boundaries with arrow marks).

The study was based on the representative plant specimens randomly collected from JU campus area in different months and seasons during 2009-2020. Besides authors' own collections, the herbarium specimens sporadically collected from this area by different collectors and deposited at Jahangirnagar University Herbarium (JUH) were also used. Taxonomic identification of the specimens was done through expert determination (JUH; Bangladesh National Herbarium, DACB), consulting the relevant taxonomic literatures (Hooker, 1872-1897; Prain, 1903; Siddiqui et al., 2007; Ahmed et al., 2008-2009; Ahmed et al., 2009; Wu et al., 1999-2013), matching with the pertinent and authentically identified voucher specimens housed at DACB and JUH, relevant type images available in the web sites of different international herbaria, such as the Royal Botanic Gardens Kew (K), Muséum National d'Histoire Naturelle France, Paris (P) and the Conservatoire et Jardin botaniques de la Ville de Genève $(\mathrm{G})$, and direct examination at JUH. All voucher specimens are preserved at JUH.

Nomenclatural information was collected from Wu and Raven (1994-2001) and Wu et al. (1999-2013), and the nomenclatural data bases (The Plant List, 2013; POWO, 2020; TROPICOS, 2021). The families of Pteridophyta have been arranged following Pichi (1977) and those of Gymnosperms and Angiosperms following Kramer and Green (1990) and Cronquist (1981), 
respectively, and the genera and species under each family alphabetically. Information on the nativeness of the species were collected consulting the literatures (Wu and Raven, 1994-2001; Hossain and Pasha, 2001; Siddiqui et al. 2007, Ahmed et al. 2008-2009, Ahmed et al. 2009; Hossain et al., 2009; Wu et al., 1999-2013) and digitized data of POWO (2020).

The common names (Bangla) were extracted from Siddiqui et al. (2007), Ahmed et al. (20082009), Ahmed et al. (2009), Pasha and Uddin (2013) and Huq (2019). The major categories of uses were recognized based on literatures (Ghani, 2003; Siddiqui et al., 2007; Ahmed et al., 20082009; Ahmed et al., 2009). The images on the study area were downloaded from Google Earth Pro 7.3.3.7786 (www.google.com). Comments on threat, conservation and management aspects have been made based on direct field observation.

\section{Results and Discussion}

During this study the campus area of Jahangirnagar University (JU) has been found to harbour a total of 917 species belonging to 574 genera and 145 families of vascular plants. The Pteridophytes, Gymnosperms and Angiosperms of this area are represented by 22, 12 and 883 species under 17, 7 and 549 genera, respectively. The division Magnoliopsida (dicotyledons) is represented by 618 species belonging to 401 genera and 102 families, whereas, the division Liliopsida (monocotyledons) by 265 species under 148 genera and 27 families (Table 1). A total of 45 families are represented by a single genus and a single species each, whereas, 25 families by at least 10 species each. In dicotyledons, Fabaceae with 44 species has been found as the largest family, which is followed by Asteraceae (with 40 species) and Rubiaceae (29 species). In monocotyledons, Poaceae is found as the largest family (with 89 species), which is followed by Cyperaceae (34 species), and Araceae and Arecaceae (24 species each).

In this area, total 515 species $(56 \%)$ are found as herbs, which are followed by 212 species of trees $(23 \%), 140$ species of shrubs (15\%), 24 species of palms (3\%), 14 species of liana (2\%) and 12 species of bamboo (1\%). The species of Magnoliopsida is composed of $44.17 \%$ herbs, $32.20 \%$ trees and $21.36 \%$ shrubs, whereas that of Liliopsida is consisted of $83.02 \%$ herbs, $9.06 \%$ palm, $4.53 \%$ bamboo, and $1.89 \%$ shrubs. All of the species enumerated here are economically or ecologically important. However, total $493(53.76 \%)$ of these species are medicinals, 318 (34.68\%) ornamentals, $83(9.05 \%)$ fodders or forages, $62(6.76 \%)$ fruits, $61(6.65 \%)$ vegetables and $46(5.02 \%)$ timbers (Table 1 and Fig. 2). Other species found in this area are economically or ecologically important in different ways, especially as firewood/fuel wood, fibre, handicrafts, spices, soil binder, green manure, dye and oil yielding species.

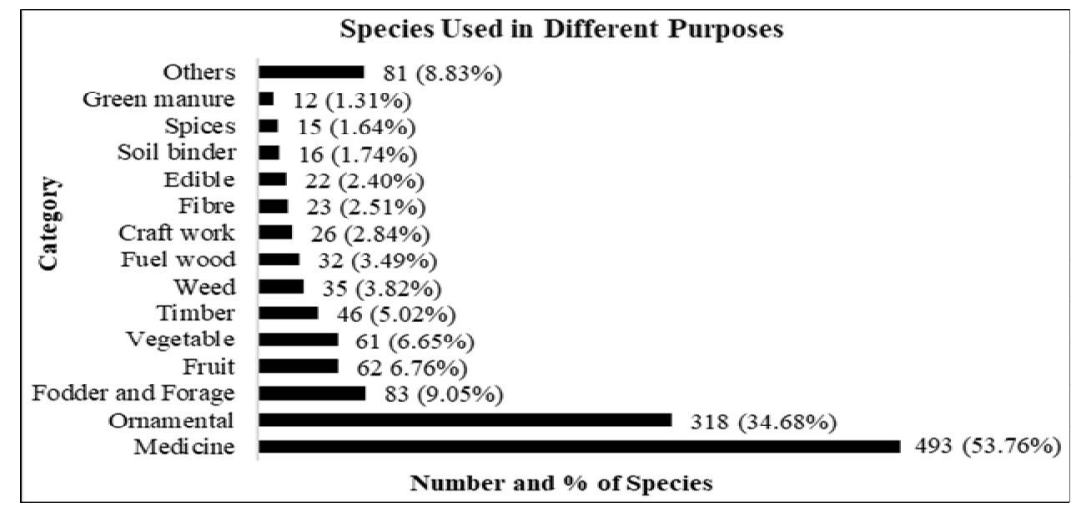

Fig. 2. Economic and ecological importance of the vascular flora of JU campus area. 
Among the species of vascular plants, total $586(63.90 \%)$ are native and $331(36.10 \%)$ are exotic to Bangladesh (Wu et al., 1999-2013; Hossain and Pasha, 2001; POWO, 2020). In Pteridophytes, Gymnosperms and Angiosperms, 90.91\%, 16.67\% and 63.76\% species, respectively, are native and $9.09 \%, 83.33 \%$ and $36.24 \%$, respectively, are exotic to Bangladesh. Many of the exotic species (Acacia auriculiformis, Ageratum conyzoides, Azolla pinnata, Chromolaena odorata, Eclipta prostrata, Eichhornia crassipes, Euphorbia hirta, Evolvulus nummularius, Hyptis suaveolens, Imperata cylindrica, Lantana camara, Mikania cordata, Mimosa pudica, Oxalis corniculata, Parthenium hysterophorus, Phyllanthus niruri, Salvinia adnata, Sphagneticola trilobata, Swietenia mahagoni, Tridax procumbens) are now completely naturalized in this area. Some of the exotic species (C. odorata, I. cylindrica, H. suaveolens, M. cordata, $P$. hysterophorus, $S$. trilobata) are found as invasive in the area. In the recent years, about 34 exotic and 24 native species have been introduced in the study area from other regions (Table 1).

In the study area, $56.27 \%$ of vascular plant species including all of the 22 species of Pteridophytes and 494 species of Angiosperms are found in wild with natural regeneration, whereas, $26.50 \%$ of the species including all of the 12 species of Gymnosperms and 231 species of Angiosperms are recorded as planted and 3.16\% of the species, consist of 29 species of Angiosperm, are appeared as cultivated. Besides, $11.45 \%$ and $2.62 \%$ of the species, comprising 129 species of Angiosperms, are originally planted and cultivated, respectively in the area, but now regenerating there naturally in wild. About $9.92 \%$ of 272 planted and cultivated species are seasonal ornamentals but visible in each year in the area. A total of $353(38.39 \%)$ species are distributed in the gardens, $313(34.13 \%)$ in the fallow lands, $284(30.86 \%)$ in roadsides, 199 $(21.70 \%)$ in the adjacent areas of residential buildings, $173(18.87 \%)$ in grasslands, $165(18.10 \%)$ in the woodlands, $152(16.68 \%)$ in open scrub jungles and $105(11.45 \%)$ in wetlands, and the rest on the lake banks, marginal lands, brick wall or on other plants (Table 1 and Fig. 3).

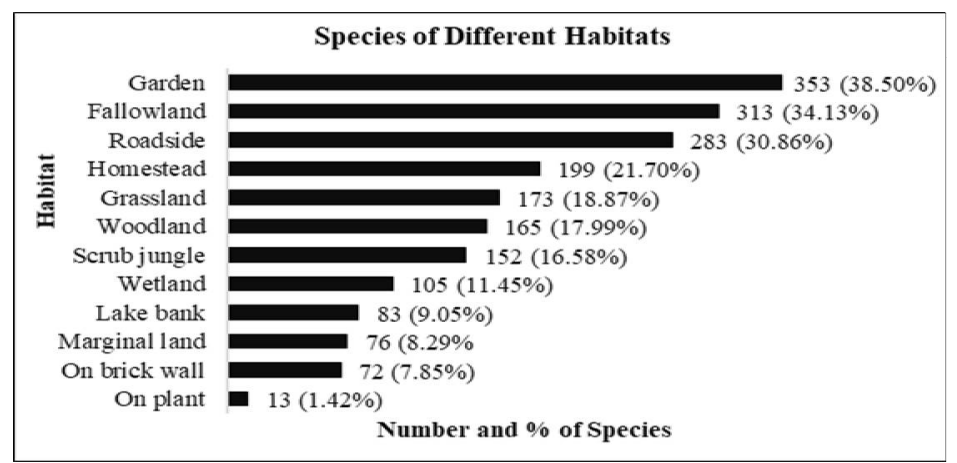

Fig. 3. Distribution of plant species in different habitats of JU campus area.

This campus area is naturally housing 15 plant species, previously listed as threatened to Bangladesh (Table 1; Ara et al., 2013; Khan et al., 2001), three (Corypha taliera, Ochna pumila and Olax nana) of which are cited as critically endangered (CR), one (Phoenix acaulis) as endangered (EN) and two (Careya herbacea and Cyperus thomsonii) as vulnerable (VU), which can be considered as the in situ conservation of these species. A comparison with the floristic composition of few campus, (semi-)urban, urban, rural and forest areas of Bangladesh, show that the study area harbours relatively higher number of plant species and thus floristically richer in respect to the size of the area (Fig. 4). Based on the results, it is concluded that a small seminatural area of a semi-urban region like this campus can be rich and diverse in plant species composition if it is maintained properly. 
Table 1. List of vascular plants of Jahangirnagar University campus, Savar, Dhaka, Bangladesh.

\begin{tabular}{|c|c|c|c|c|c|}
\hline Scientific name & Bangla name & Habitat & Habit & Origin RSE & Use \\
\hline \multicolumn{6}{|l|}{ PTERIDOPHYTA Schimp. } \\
\hline \multicolumn{6}{|l|}{ Selaginellaceae Willk. } \\
\hline Selaginella ciliaris (Retz.) Spring & Katagenella & $\mathrm{Fl}, \mathrm{Rs}$ & Herb, cr, w & Native $M A R 2812$ & $\mathrm{M}, \mathrm{O}$ \\
\hline S. vaginata Spring & Nataginella & $\mathrm{Fl}, \mathrm{Rs}$ & Herb, cr, w & Native $M A R 2813$ & $\mathrm{M}, \mathrm{O}$ \\
\hline \multicolumn{6}{|l|}{ Ophioglossaceae Martinov } \\
\hline Ophioglossum reticulatum $\mathrm{L}$. & Sharpa jhibba & $\mathrm{Fl}, \mathrm{Lb}, \mathrm{Wtl}$ & Herb, er; w & Native $G M H 5145$ & M \\
\hline \multicolumn{6}{|l|}{ Salviniaceae Martinov } \\
\hline Azolla pinnata $\mathrm{R} . \mathrm{Br}$. & Khudipana & Wtl & Herb, ff; w & Exotic GMH 5140 & $\mathrm{Gm}$ \\
\hline Salvinia adnata Desv. & Chaptakani & Wtl & Herb, ff; w & Exotic GMH 5141 & Ap, O \\
\hline S. cucullata Roxb. & Indur kani & Wtl & Herb, ff; w & Native GMH 5142 & Ap, O \\
\hline S. natans (L.) All. & Bhashan pata & Wtl & Herb, ff; w & Native $G M H 5143$ & Ap, O \\
\hline \multicolumn{6}{|l|}{ Marsileaceae Mirb. } \\
\hline Marsilea minuta $\mathrm{L}$. & Susni shak & $\mathrm{Fl}, \mathrm{Lb}, \mathrm{Wtl}$ & Herb, cr; w & Native SSS 2169 & $\mathrm{M}, \mathrm{Vg}$ \\
\hline \multicolumn{6}{|l|}{ Lygodiaceae M. Roem. } \\
\hline Lygodium flexuosum (L.) Sw. & Saralata fern & $\mathrm{Fl}, \mathrm{Rs}, \mathrm{Sj}$ & Herb, vi; w & Native GMH 5144 & M \\
\hline \multicolumn{6}{|l|}{ Pteridaceae E.D.M. Kirchn. } \\
\hline Adiantum capillus-veneris L. & Venichadda & $\mathrm{Bw}^{1}, \mathrm{Rs}, \mathrm{Gr}, \mathrm{Hs}$ & Herb, pr; w & Native SSS 2171 & $\mathrm{O}$ \\
\hline A. philippense $\mathrm{L}$. & Kalijhat & $\mathrm{Gr}, \mathrm{Lb}$ & Herb, pr; w & Native $G M H 5146$ & $\mathrm{O}$ \\
\hline Ceratopteris thalictroides (L.) Brongn. & Pani lettuce & Fl. Lb, Wtl & Herb, er; w & Native SSS 2173 & $\mathrm{O}$ \\
\hline Hemionitis belangeri (Bory) Christenh. & Belangiri fern & $\mathrm{Lb}, \mathrm{Rs}$ & Herb, er; w & Native SSS 2172 & M \\
\hline Pteris vittata $\mathrm{L}$. & Imodi pteris & $\mathrm{Bw}^{1}$ & Herb, pr; w & Native GMH 5147 & $\mathrm{O}$ \\
\hline \multicolumn{6}{|l|}{ Lindsaeaceae C. Presl ex M.R. Schomb. } \\
\hline Lindsaea ensifolia $\mathrm{Sw}$. & Lindoli dheki & $\mathrm{Fl}, \mathrm{Rs}$ & Herb, er; w & Native GMH 5148 & $\mathrm{O}$ \\
\hline \multicolumn{6}{|l|}{ Polypodiaceae J. Presl \& C. Presl } \\
\hline Drynaria quercifolia (L.) J. Sm. & Pankhiraj & $\mathrm{Bw}^{1}, \mathrm{Hs}, \mathrm{Op}$ & Herb, ep; w & Native GMH 5152 & M \\
\hline Microsorum punctatum (L.) Copel. & Punctasorum fern & $\mathrm{Bw}^{1}, \mathrm{Op}$ & Herb, ep; w & Native GMH 5153 & $\mathrm{O}$ \\
\hline Pyrrosia lanceolata (L.) Farw. & Atashirossi & Op & Herb, ep; w & Native SSS 2175 & $\mathrm{O}$ \\
\hline P. nuda (Giesenh.) Ching & Nudarossi & Op & Herb, ep; w & Native $G M H 5154$ & $\mathrm{O}$ \\
\hline \multicolumn{6}{|l|}{ Thelypteridaceae Ching ex Pic. Serm. } \\
\hline Ampelopteris prolifera (Retz.) Copel. & $\begin{array}{l}\text { Lombo dheki } \\
\text { shak }\end{array}$ & $\mathrm{Fl}, \mathrm{Ml}, \mathrm{Lb}$ & Herb, cr; w & Native GMH 5149 & $\mathrm{Vg}$ \\
\hline $\begin{array}{l}\text { Thelypteris dentata (Forssk.) E.P. } \\
\text { St.John }\end{array}$ & Datitila & $\mathrm{Bw}^{1}, \mathrm{Fl}, \mathrm{Ml}, \mathrm{Lb}$ & Herb, cr; w & Native GMH 5150 & $\mathrm{Vg}$ \\
\hline \multicolumn{6}{|l|}{ Athyriaceae Alston } \\
\hline Diplazium esculentum (Retz.) Sw. & Dheki shak & $\mathrm{Fl}, \mathrm{Lb}, \mathrm{Rs}$ & Herb, er; w & Native GMH 5151 & $\mathrm{Vg}$ \\
\hline \multicolumn{6}{|l|}{ GYMNOSPERMAE Prantl } \\
\hline \multicolumn{6}{|l|}{ Cycadaceae Pers. } \\
\hline Cycas circinalis $\mathrm{L}$. & Nali cycas & $\mathrm{Gr}, \mathrm{Hs}$ & Tree, $\mathrm{m} ; \mathrm{pl}$ & Exotic GMH 5156 & $\mathrm{M}, \mathrm{O}$ \\
\hline C. pectinata Buch.-Ham.\# & Moni raj & $\mathrm{Gr}, \mathrm{Hs}$ & Tree, $\mathrm{m} ; \mathrm{pl}$ & Native GMH 5155 & $\mathrm{M}, \mathrm{O}$ \\
\hline C. revoluta Thunb. & Volu cycas & $\mathrm{Gr}, \mathrm{Hs}$ & Tree, $\mathrm{m} ; \mathrm{pl}$ & Exotic GMH 5157 & $\mathrm{O}$ \\
\hline \multicolumn{6}{|l|}{ Zamiaceae Horan. } \\
\hline Zamia furfuracea L. f. ex Aiton + & Unknown & $\mathrm{Gr}$ & Shrub; pl & Exotic MAR 2814 & $\mathrm{O}$ \\
\hline Z. pumila L. + & Zamia cycad & $\mathrm{Gr}$ & Shrub; pl & Exotic MAR 2815 & $\mathrm{O}$ \\
\hline
\end{tabular}


Table 1 contd.

\begin{tabular}{|c|c|c|c|c|c|}
\hline Scientific name & Bangla name & Habitat & Habit & Origin RSE & Use \\
\hline \multicolumn{6}{|l|}{ Podocarpaceae Endl. } \\
\hline Podocarpus neriifolius D. Don & Bash pata & $\mathrm{Gr}$ & Tree, $\mathrm{m} ; \mathrm{pl}$ & Native $G M H 5158$ & $\mathrm{M}, \mathrm{T}$ \\
\hline \multicolumn{6}{|l|}{ Araucariaceae Henkel \& W. Hochst. } \\
\hline Araucaria heterophylla (Salisb.) Franco & Dipati aurocaria & Gr, Hs, Rs & Tree, $1 ; \mathrm{pl}$ & Exotic SSS 2176 & $\mathrm{O}$ \\
\hline \multicolumn{6}{|l|}{ Cupressaceae Gray } \\
\hline Juniperus chinensis $\mathrm{L}$. & China Juniper & Rs & Tree, s; pl & Exotic MAR 2716 & $\mathrm{O}$ \\
\hline Thuja plicata Donn ex D. Don & Thuja & Gr, Hs & Tree, $1 ; \mathrm{pl}$ & Exotic SSS 2177 & $\mathrm{O}, \mathrm{T}$ \\
\hline Platycladus orientalis (L.) Franco & Thuja jhau & $\mathrm{Gr}, \mathrm{Hs}$ & Shrub; pl & Exotic SSS 2178 & $\mathrm{O}$ \\
\hline \multicolumn{6}{|l|}{ Pinaceae Spreng. ex Rudolphi } \\
\hline Pinus caribaea Morelet & Pine gach & Gr, Rs & Tree, $1 ; \mathrm{pl}$ & Exotic SSS 2179 & $\mathrm{M}, \mathrm{T}$ \\
\hline P. palustris Mill. & Pine & Gr, Rs & Tree, $1 ; \mathrm{pl}$ & Exotic SSS 2180 & M \\
\hline \multicolumn{6}{|l|}{ MAGNOLIOPSIDA Brongn. } \\
\hline \multicolumn{6}{|l|}{ Magnoliaceae Juss. } \\
\hline Magnolia champaca (L.) Baill. ex Pierre & Champa & Gr, Rs & Tree, $1 ; \mathrm{pl}$ & Native $S A K 2022$ & $\mathrm{M}, \mathrm{O}$ \\
\hline M. grandiflora $\mathrm{L}$. & Udoy poddo & $\mathrm{Gr}, \mathrm{Hs}$ & Tree, $\mathrm{m} ; \mathrm{pl}$ & Exotic $S A K 2148$ & $\mathrm{M}, \mathrm{O}$ \\
\hline \multicolumn{6}{|l|}{ Annonaceae Juss. } \\
\hline Annona reticulata $\mathrm{L}$. & Atta & $\mathrm{Gr}, \mathrm{Hs}, \mathrm{Fl}$ & Tree, s; pl-w & Exotic GMH 5007 & $\mathrm{Fr}, \mathrm{Fw}$ \\
\hline A. squamosa $\mathrm{L}$. & Shorifa & $\mathrm{Gr}, \mathrm{Hs}$ & Tree, s; pl & Exotic GMH 5002 & $\mathrm{Fr}$ \\
\hline Artabotrys hexapetalus (L.f.) Bhandari & Kathali chapma & $\mathrm{Gr}, \mathrm{Hs}$ & Shrub, sc; pl & Exotic GMH 5005 & $\mathrm{M}, \mathrm{O}$ \\
\hline $\begin{array}{l}\text { Huberantha pendula (Capuron ex G.E. } \\
\text { Schatz \& Le Thomas) Chaowasku }\end{array}$ & Weeping debdaru & Rs & Tree, $\mathrm{m} ; \mathrm{pl}$ & Exotic MAR 2530 & $\mathrm{Fu}, \mathrm{O}$ \\
\hline $\begin{array}{l}\text { Miliusa velutina (Dunal) Hook. f. \& } \\
\text { Thomson }\end{array}$ & Gandhi gojari & Wl & Tree, $1 ; \mathrm{pl}$ & Native MAR 123 & $\mathrm{~T}, \mathrm{M}$ \\
\hline Polyalthia longifolia (Sonn.) Thwaites & Debdaru & Rs, Wl & Tree, $1 ; \mathrm{pl}-\mathrm{w}$ & Exotic MAR 132 & $\mathrm{O}, \mathrm{T}$ \\
\hline P. suberosa (Roxb.) Thwaites & Barachali & $\mathrm{Fl}, \mathrm{Wl}$ & Tree, s; w & Native $S S 358$ & Fr, M \\
\hline \multicolumn{6}{|l|}{ Lauraceae Juss. } \\
\hline Cinnamomum camphora (L.) J. Presl + & Karpur & $\mathrm{Gr}, \mathrm{Hs}$ & Tree, $\mathrm{m} ; \mathrm{pl}$ & Exotic SAK 2005 & $\mathrm{Co}, \mathrm{M}$ \\
\hline $\begin{array}{l}\text { C. tamala (Buch.-Ham.) T. Nees \& } \\
\text { Eberm. }\end{array}$ & Tejpata & $\mathrm{Gr}, \mathrm{Hs}$ & Tree, $\mathrm{m} ; \mathrm{pl}$ & Native $S A K 2049$ & $\mathrm{Co}, \mathrm{Sp}$ \\
\hline C. verum J. Presl & Darchini & $\mathrm{Gr}, \mathrm{Hs}$ & Tree, $\mathrm{m} ; \mathrm{pl}$ & Exotic SAK 2018 & $\mathrm{Sp}$ \\
\hline Litsea glutinosa (Lour.) C.B. Rob. & Kukurchita & $\mathrm{Sj}, \mathrm{Wl}$ & Tree, $\mathrm{m} ; \mathrm{w}$ & Native $S A K 2046$ & M \\
\hline L. monopetala (Roxb.) Pers. & Boro-kukurchita & $\mathrm{Sj}, \mathrm{Wl}$ & Tree, $\mathrm{m} ; \mathrm{w}$ & Native $S A K 2047$ & M \\
\hline Persea americana Mill. + & Avocado & $\mathrm{Gr}$ & Tree, $\mathrm{m} ; \mathrm{pl}$ & Exotic SAK 2004 & $\mathrm{Fr}$ \\
\hline \multicolumn{6}{|l|}{ Piperaceae Giseke } \\
\hline Peperomia pellucida (L.) Kunth & Luchi pata & $\mathrm{Bw}^{1}, \mathrm{Ml}, \mathrm{Wl}$ & Herb, pr; w & Exotic SS 425 & M \\
\hline Piper betle $\mathrm{L}$. & Pan & Gr, Hs & Herb, vi; cv & Exotic MAR 2531 & M \\
\hline P. longum $\mathrm{L}$. & Pipul & $\mathrm{Fl}, \mathrm{Wl}$ & Herb, vi; w & Native $S S 426$ & M \\
\hline P. nigrum $\mathrm{L}$. & Golmorich & $\mathrm{Gr}, \mathrm{Hs}$ & Herb, vi; cv & Exotic MAR 2532 & $\mathrm{Sp}$ \\
\hline P. sarmentosum Roxb. & Jongli pan & $\mathrm{Gr}, \mathrm{Sj}$ & Herb, cr, cv-w & Exotic MAR 2533 & M \\
\hline \multicolumn{6}{|l|}{ Aristolochiaceae Juss. } \\
\hline Aristolochia indica $\mathrm{L}$. & Ishwarmul & $\mathrm{Sj}$ & Herb, cr; w & Native $M A R 140$ & M \\
\hline \multicolumn{6}{|l|}{ Nelumbonaceae A. Rich. } \\
\hline $\begin{array}{l}\text { Nelumbo nucifera Gaertn. } \\
\text { Nymphaeaceae Salisb. }\end{array}$ & Poddo & Wtl & Herb, fr; cv-w & Native $M A R 2534$ & $\mathrm{M}, \mathrm{O}$ \\
\hline Nymphaea nouchali Burm. f. & Nil shapla & Wtl & Herb, fr; w & Native SS 397 & $\mathrm{M}, \mathrm{O}$ \\
\hline
\end{tabular}


Table 1 contd.

\begin{tabular}{|c|c|c|c|c|c|}
\hline Scientific name & Bangla name & Habitat & Habit & Origin RSE & Use \\
\hline N. pubescens Willd. & Sada shapla & Wtl & Herb, fr; w & Native $M A R 134$ & $\mathrm{O}, \mathrm{Vg}$ \\
\hline N. rubra Roxb. ex Andrews & Lal shapla & Wtl & Herb, fr; w & Native $S S 427$ & $\mathrm{O}, \mathrm{Vg}$ \\
\hline \multicolumn{6}{|l|}{ Ceratophyllaceae Gray } \\
\hline Ceratophyllum demersum $\mathrm{L}$. & Kantajhanjhi & Wtl & Herb, sm; w & Native $M A R 173$ & M \\
\hline \multicolumn{6}{|l|}{ Ranunculaceae Juss. } \\
\hline Clematis gouriana Roxb. ex DC. & Chagalbati & $\mathrm{Sj}$ & Herb, er; w & Native $S S 428$ & M \\
\hline Ranunculus sceleratus L. & Palik & Wtl & Herb, er; w & Native MAR 2535 & M, Po \\
\hline \multicolumn{6}{|l|}{ Menispermaceae Juss. } \\
\hline Cocculus hirsutus (L.) W. Theob. & Huyer & $\mathrm{Sj}$ & Herb, vi; w & Native SS 191 & M \\
\hline Stephania glabra (Roxb.) Miers & Thandamanik & $\mathrm{Sj}$ & Herb, vi; w & Native $M A R 126$ & M \\
\hline S. japonica (Thunb.) Miers & Akandi manik & $\mathrm{Sj}$ & Herb, vi; w & Native MAR 2536 & M \\
\hline $\begin{array}{l}\text { Tinospora crispa (L.) Hook. f. \& } \\
\text { Thomson }\end{array}$ & Gulancha & $\mathrm{Wl}$ & Herb, vi; w & Native SS 399 & M \\
\hline T. sinensis (Lour.) Merr. & Padma gulancha & Wl & Shrub, sc; w & Native MAR 2537 & M \\
\hline Tiliacora racemosa Colebr. & Baghlata & $\mathrm{Fl}, \mathrm{Wl}$ & Shrub, sc; w & Native $M A R 2538$ & M \\
\hline \multicolumn{6}{|l|}{ Cannabaceae Martinov } \\
\hline Trema orientalis (L.) Blume & Banjiga & $\mathrm{Bw}^{2}, \mathrm{Sj}, \mathrm{Wl}$ & Tree, m; w & Native SS187 & $\mathrm{FW}_{\mathrm{W}}$ \\
\hline \multicolumn{6}{|l|}{ Moraceae Gaudich. } \\
\hline $\begin{array}{l}\text { Artocarpus altilis (Parkinson ex F.A. } \\
\text { Zorn) Fosberg + }\end{array}$ & Bread fruit & $\mathrm{Gr}$ & Tree, $\mathrm{m} ; \mathrm{pl}$ & Exotic MAR 2539 & Fr \\
\hline A. chama Buch.-Ham. & Chapalish & Wl, Rs & Tree, 1; pl-w & Native MAR 166 & $\mathrm{Fr}, \mathrm{T}$ \\
\hline A. heterophyllus Lam. & Kanthal & Wl, Hs & Tree, m; pl-w & Exotic $S S 429$ & $\mathrm{Fr}, \mathrm{T}$ \\
\hline A. lakoocha Roxb. & Dewa & $\mathrm{Wl}, \mathrm{Gr}$ & Tree, m; pl-w & Native MAR 2540 & Fr, Fw \\
\hline Ficus benghalensis $\mathrm{L}$. & Bot & $\mathrm{Bw}^{2}, \mathrm{Wl}$ & Tree, l; w & Native $S S 430$ & Fw, Sd \\
\hline F. benjamina $\mathrm{L}$. & Jiri pakur & $\mathrm{Gr}$ & Tree, $\mathrm{m} ; \mathrm{pl}$ & Native MAR 2541 & Fw, O \\
\hline F. elastica Roxb. ex Hornem. & Rubber bot & Gr, Rs & Tree, m; pl & Native MAR 2542 & $\mathrm{O}$ \\
\hline F. heterophylla L. f. & Bhuidumur & $\mathrm{Sj}, \mathrm{Wl}$ & Shrub; w & Native SS 431 & Fw, M \\
\hline F. hispida L. f. & Kakdumur & $\mathrm{Bw}^{2}, \mathrm{Sj}, \mathrm{Wl}$ & Tree, s; w & Native SS 262 & $\mathrm{M}, \mathrm{Vg}$ \\
\hline F. pumila $\mathrm{L}$. & Latabot & $\mathrm{Bw}^{1}$ & Herb, cr; pl-w & Exotic $M A R 72$ & $\mathrm{O}$ \\
\hline F. racemosa $\mathrm{L}$. & Jagdumur & $\mathrm{Bw}^{2}, \mathrm{Sj}, \mathrm{Fl}$ & Tree, l; w & Native $M A R 73$ & Fr, M \\
\hline F. religiosa $\mathrm{L}$. & Ashwath & $\mathrm{Bw}^{2}, \mathrm{Wl}, \mathrm{Sj}$ & Tree, 1 ; w & Native SS 432 & $\mathrm{O}, \mathrm{Sd}$ \\
\hline F. rumphii Blume & Khiri bot & $\mathrm{Wl}$ & Tree, l; w & Native $M A R 2543$ & $\mathrm{O}, \mathrm{Sd}$ \\
\hline Morus alba L. + & Shada tut & Hs, Ml, Rs & Tree, s; pl & Exotic MAR 2544 & $\mathrm{Fr}, \mathrm{Sf}$ \\
\hline Streblus asper Lour. & Sheora & $\mathrm{Sj}, \mathrm{Wl}$ & Tree, 1 ; w & Native SS 193 & Fw, M \\
\hline \multicolumn{6}{|l|}{ Urticaceae Juss. } \\
\hline Gonostegia hirta (Blume ex Hassk.) Miq. & Unknown & $\mathrm{Fl}, \mathrm{Ml}, \mathrm{Wtl}$ & Herb, er; w & Native $M A R 2545$ & M \\
\hline Laportea interrupta (L.) Chew & Chotrapatta & $\mathrm{Sj} W \mathrm{l}$ & Herb, er; w & Native MAR 2545 & M, Po \\
\hline Pilea microphylla (L.) Liebm. & Latamaricha & $\mathrm{Bw}^{1}$ & Herb, pr; w & Exotic MAR 100 & M \\
\hline Pouzolzia zeylanica (L.) Benn. & Kullaruki & $\mathrm{Bw}^{1}, \mathrm{Sj}, \mathrm{Gl}$ & Herb, er; w & Native $M A R 165$ & M \\
\hline \multicolumn{6}{|l|}{ Casuarinaceae R. Br. } \\
\hline Casuarina equisetifolia $\mathrm{L}$. & Jhau & Gr, Rs & Tree, $1 ; \mathrm{pl}$ & Native SSS 2003 & $\mathrm{O}$ \\
\hline \multicolumn{6}{|l|}{ Nyctaginaceae Juss. } \\
\hline Boerhavia diffusa $\mathrm{L}$. & Punarnava & $\mathrm{Bw}^{1}, \mathrm{Fl}, \mathrm{Sj}$ & Herb, pr; w & Native SS 396 & M \\
\hline Bougainvillea buttiana Holttum \& Standl. & Halud baganbilas & $\mathrm{Gr}, \mathrm{Hs}$ & Shrub, sc; pl & Exotic SSS 2004 & $\mathrm{O}$ \\
\hline B. glabra Choisy & Baganbilas & $\mathrm{Gr}$ & Shrub, sc; pl & Exotic SSS 2005 & $\mathrm{O}$ \\
\hline
\end{tabular}


Table 1 contd.

\begin{tabular}{|c|c|c|c|c|c|}
\hline Scientific name & Bangla name & Habitat & Habit & Origin RSE & Use \\
\hline B. peruviana Bonpl. & $\begin{array}{l}\text { Shuvro } \\
\text { baganbilas }\end{array}$ & $\mathrm{Gr}, \mathrm{Hs}$ & Shrub, sc; pl & Exotic SSS 2006 & $\mathrm{O}$ \\
\hline B. spectabilis Willd. & Shukhbilas & $\mathrm{Gr}, \mathrm{Hs}$ & Shrub, sc; pl & Exotic SSS 2007 & $\mathrm{O}$ \\
\hline Mirabilis jalapa $\mathrm{L}$. & Sandhyamoni & $\mathrm{Sj}, \mathrm{Gr}, \mathrm{Hs}$ & Herb, er; pl-w & Exotic SSS 2008 & $\mathrm{M}, \mathrm{O}$ \\
\hline \multicolumn{6}{|l|}{ Cactaceae Juss. } \\
\hline Epiphyllum oxypetalum (DC.) Haw. & Night queen & Gr & Herb, pr; pl & Exotic GMH 5161 & $\mathrm{O}$ \\
\hline Selenicereus undatus (Haw.) D.R. Hun + & Dragan phal & Gr & Herb, vi; pl & Exotic GMH 5160 & $\mathrm{Fr}$ \\
\hline Opuntia ficus-indica (L.) Mill. & Fonimonosha & Ml, Rs & Shrub; w & Exotic MAR 2710 & $\mathrm{He}, \mathrm{M}$ \\
\hline O. stricta (Haw.) Haw. & Nagphana & Ml, Rs & Shrub; w & Exotic $M A R 2711$ & $\mathrm{He}, \mathrm{M}$ \\
\hline \multicolumn{6}{|l|}{ Amaranthaceae Juss. } \\
\hline Achyranthes aspera $\mathrm{L}$. & Apang & $\mathrm{Gl}, \mathrm{Fl}, \mathrm{Wl}$ & Herb, er; w & Native $S S 124$ & M \\
\hline Alternanthera paronychioides A. St.-Hil. & Jhuli khata & $\mathrm{Gl}, \mathrm{Fl}, \mathrm{Rs}$ & Herb, pr; w & Exotic MAR 2547 & $\mathrm{Vg}$ \\
\hline A. philoxeroides (Mart.) Griseb. & Henchi & Wtl & Herb, fr; w & Exotic $S S 353$ & $\mathrm{Vg}$ \\
\hline A. sessilis (L.) R. Br. ex DC. & Malancha & $\mathrm{Bw}^{1}, \mathrm{Gl}, \mathrm{Fl}, \mathrm{Rs}$ & Herb, pr; w & Exotic MAR 174 & $\mathrm{Vg}$ \\
\hline Amaranthus blitum L. & Goburanotey & Gl, Rs & Herb, er; w & Exotic SS 355 & $\mathrm{Vg}$ \\
\hline A. graecizans $\mathrm{L}$. & Unknown & Gl, Rs & Herb, er; w & Native $S S 354$ & $\mathrm{Vg}$ \\
\hline A. spinosus $\mathrm{L}$. & Kantanotey & Gl, Fl, Rs & Herb, er; w & Exotic SS 120 & $\mathrm{M}, \mathrm{Vg}$ \\
\hline A. tricolor $\mathrm{L}$. & Lalshak & Hs, Fl, Rs & Herb, er; pl-w & Native $M A R 2548$ & $\mathrm{Vg}$ \\
\hline A. viridis $\mathrm{L}$. & Notey shak & $\mathrm{Fl}, \mathrm{Rs}$ & Herb, er; w & Exotic SS 133 & $\mathrm{Vg}$ \\
\hline Celosia argentea $\mathrm{L}$. & Morog phul & Hs, Rs & Herb, er; pl-w & Exotic SS 356 & $\mathrm{M}, \mathrm{O}$ \\
\hline Chenopodium album $\mathrm{L}$. & Botua shak & $\mathrm{Fl}, \mathrm{Gr}, \mathrm{Rs}$ & Herb, er; w & Native $G M H 2151$ & $\mathrm{M}, \mathrm{Vg}$ \\
\hline Cyathula prostrata $(\mathrm{L}$.) Blume & Shyontula & $\mathrm{Gl}, \mathrm{Fl}, \mathrm{Rs}$ & Herb, pr; w & Native $S S 155$ & M \\
\hline Deeringia amaranthoides (Lam.) Merr. & Golamohani & $\mathrm{Sj}$ & Herb, vi; w & Native $M A R 2549$ & $\mathrm{M}, \mathrm{Vg}$ \\
\hline Gomphrena celosioides Mart. & Golkamal & $\mathrm{Gl}, \mathrm{Fl}, \mathrm{Rs}$ & Herb, pr; w & Exotic MAR 2550 & M \\
\hline G. globosa L. & Botam phul & Hs, Rs & Herb, er; pl & Exotic $M A R 2551$ & $\mathrm{O}$ \\
\hline \multicolumn{6}{|l|}{ Portulacaceae Juss. } \\
\hline Portulaca oleracea $\mathrm{L}$. & Boronunia & $\mathrm{Bw}^{1}, \mathrm{Fl}, \mathrm{Rs}$ & Herb, pr; w & Exotic SS 446 & $\mathrm{M}, \mathrm{Vg}$ \\
\hline P. grandiflora Hook. & Time phul & Hs, Rs & Herb, pr; pl & Exotic GMH 5169 & $\mathrm{O}$ \\
\hline P. quadrifida $\mathrm{L}$. & Chhoto nunia & Hs, Rs & Herb, pr; pl-w & Native $M A R 2552$ & $\mathrm{O}$ \\
\hline \multicolumn{6}{|l|}{ Basellaceae Raf. } \\
\hline Basella alba $\mathrm{L}$. & Pui shak & $\mathrm{Hs}, \mathrm{Fl}$ & Herb, cr; pl-w & Native $M A R 2553$ & $\mathrm{Vg}$ \\
\hline \multicolumn{6}{|l|}{ Molluginaceae Bartl. } \\
\hline Glinus oppositifolius (L.) Aug. DC. & Gima shak & $\mathrm{Fl}$ & Herb, pr; w & Native GMH 5017 & $\mathrm{Vg}$ \\
\hline Trigastrotheca pentaphylla (L.) Thulin & Julpapra & $\mathrm{Fl}$ & Herb, pr; w & Exotic GMH 5019 & M \\
\hline \multicolumn{6}{|l|}{ Caryophyllaceae Juss. } \\
\hline $\begin{array}{l}\text { Polycarpon prostratum (Forssk.) Asch. } \\
\& \text { Schweinf. }\end{array}$ & Ghima & $\mathrm{Gl}, \mathrm{Fl}$ & Herb, pr; w & Native SSS 2009 & M \\
\hline \multicolumn{6}{|l|}{ Polygonaceae Juss. } \\
\hline Antigonon leptopus Hook. \& Arn. & Valobasha lata & Gr & Herb, vi; pl & Exotic $M A R 2554$ & M \\
\hline Persicaria barbata (L.) H.Hara & Biskatali & $\mathrm{Fl}, \mathrm{Wtl}$ & Herb, er; w & Native SSS 2010 & M \\
\hline P. glabra (Willd.) M.Gómez & Lal kukri & $\mathrm{Fl}$ & Herb, er; w & Native MAR 124 & M \\
\hline P. hydropiper (L.) Delarbre & Panibishkatali & $\mathrm{Fl}, \mathrm{Wtl}$ & Herb, er; w & Native $S S 270$ & M \\
\hline P. lapathefolia (L.) Delarbre & Panimarich & $\mathrm{Fl}, \mathrm{Wtl}$ & Herb, er; w & Native SS 398 & $\mathrm{O}$ \\
\hline P. minor (Huds.) Opiz & Chhoto-bishkatali & $\mathrm{Fl}$ & Herb, er; w & Native MAR 135 & M \\
\hline P. orientalis (L.) Spach & Bara panimarich & $\mathrm{Fl}, \mathrm{Wtl}$ & Herb, er; w & Native MAR 167 & M \\
\hline
\end{tabular}


Table 1 contd,.

\begin{tabular}{|c|c|c|c|c|c|}
\hline Scientific name & Bangla name & Habitat & Habit & Origin RSE & Use \\
\hline Polygonum effusum Meisn. & Rani phul & Wtl & Herb, er; w & Native SSS 2011 & $\mathrm{M}$ \\
\hline P. plebeium $\mathrm{R}$. Br. & Khudi-bishkatali & $\mathrm{Fl}$ & Herb, er; w & Native $S S 433$ & $\mathrm{M}, \mathrm{Vg}$ \\
\hline Rumex dentatus $\mathrm{L}$. & Bon-palang & $\mathrm{Fl}$ & Herb, er; w & Native SSS 2012 & M \\
\hline R. maritimus $\mathrm{L}$. & Dati-palang & $\mathrm{Fl}$ & Herb, er; w & Native SSS 2013 & M \\
\hline \multicolumn{6}{|l|}{ Dilleniaceae Salisb. } \\
\hline Dillenia indica $\mathrm{L}$. & Chalta & Wl & Tree, m; pl & Native $S S 437$ & Fr, M \\
\hline \multicolumn{6}{|l|}{ Ochnaceae DC. } \\
\hline Ochna pumila Buch.-Ham. ex DC. \# & Bhui chapa & Wl & Herb, er; w & Native MAR 107 & Fr, Po \\
\hline O. jabotapita $\mathrm{L}$. & Konok chapa & $\mathrm{Gr}$ & Tree, s; pl & Native $M A R 2555$ & M \\
\hline \multicolumn{6}{|l|}{ Dipterocarpaceae Blume } \\
\hline Dipterocarpus turbinatus Gaertn. f. & Teligarjan & Wl, Rs & Tree, 1; pl-w & Native $S S 438$ & $\mathrm{~T}$ \\
\hline Hopea odorata Roxb. & Telsur & Wl, Rs & Tree, 1; pl-w & Native SS 439 & $\mathrm{~T}$ \\
\hline Shorea robusta Roxb. & Sal & $\mathrm{Wl}$ & Tree, $1 ; \mathrm{w}$ & Native $S S 440$ & $\mathrm{~T}$ \\
\hline \multicolumn{6}{|l|}{ Theaceae Mirb. } \\
\hline Camellia japonica $\mathrm{L}$. & Camellia & $\mathrm{Gr}$ & Shrub; pl & Exotic $M A R 2558$ & $\mathrm{O}$ \\
\hline \multicolumn{6}{|l|}{ Clusiaceae Lindl. } \\
\hline Mesua ferrea $\mathrm{L}$. & Nageshawr & Gr, Rs & Tree, s; pl & Native $S S 441$ & $\mathrm{M}, \mathrm{O}$ \\
\hline Garcinia cowa Roxb. ex Choisy ++ & Cawphal & $\mathrm{Gr}, \mathrm{Hs}$ & Tree, $\mathrm{m} ; \mathrm{pl}$ & Native $M A R 2556$ & $\mathrm{Fr}$ \\
\hline $\begin{array}{l}\text { G. xanthochymus Hook. f. ex T. } \\
\text { Anderson ++ }\end{array}$ & Dephal & $\mathrm{Gr}$ & Tree, $\mathrm{m} ; \mathrm{pl}$ & Native MAR 2557 & $\mathrm{Fr}$ \\
\hline \multicolumn{6}{|l|}{ Hypericaceae Juss. } \\
\hline Hypericum japonicum Thunb. & Basanta & $\mathrm{Fl}, \mathrm{Wtl}$ & Herb, pr; w & Native $G M H 5010$ & M \\
\hline \multicolumn{6}{|l|}{ Elaeocarpaceae Juss. } \\
\hline Elaeocarpus floribundus Blume & Jalpai & $\mathrm{Gr}, \mathrm{Hs}, \mathrm{Wl}$ & Tree, $\mathrm{m} ; \mathrm{pl}$ & Native MAR 2620 & $\mathrm{Fr}, \mathrm{Oy}$ \\
\hline \multicolumn{6}{|l|}{ Sterculiaceae Vent. } \\
\hline Abroma augusta (L.) L. f. & Ulatkambal & $\mathrm{Hs}, \mathrm{Sj}$ & Shrub; pl-w & Native SS 434 & $\mathrm{Fb}, \mathrm{M}$ \\
\hline Helicteres isora $\mathrm{L}$. & Atmora & $\mathrm{Sj}, \mathrm{Wl}$ & Shrub; w & Native $S S 435 a$ & $\mathrm{Fb}, \mathrm{M}$ \\
\hline Melochia corchorifolia $\mathrm{L}$. & Tiki-okra & Wl & Shrub; w & Native MAR 151 & M \\
\hline Sterculia foetida $\mathrm{L}$. & Baksho badam & Gr, Rs & Tree, $1 ; \mathrm{pl}$ & Native $G M H 5027$ & $\mathrm{Fr}, \mathrm{O}$ \\
\hline S. villosa Roxb. & Udal & Gr, Wl & Tree, 1; pl-w & Native $G M H 5015$ & $\mathrm{M}, \mathrm{Pp}$ \\
\hline \multicolumn{6}{|l|}{ Bombacaceae Kunth. } \\
\hline Bombax ceiba $\mathrm{L}$. & Shimul & $\mathrm{Wl}, \mathrm{Sj}$ & Tree, 1 ; w & Native $S S 368$ & $\mathrm{Fb}, \mathrm{M}$ \\
\hline \multicolumn{6}{|l|}{ Malvaceae Juss. } \\
\hline Abelmoschus esculentus (L.) Moench & Dherosh & $\mathrm{Hg}$ & Herb, er; cv & Native $M A R 2561$ & $\mathrm{Vg}$ \\
\hline A. moschatus Medik. & Mushokdana & $\mathrm{Sj}, \mathrm{Fl}$ & Herb, er; w & Native SS 443 & M \\
\hline Abutilon indicum (L.) Sweet & Petari & $\mathrm{Fl}, \mathrm{Rs}, \mathrm{Sj}$ & Shrub; w & Native SSS 2015 & M \\
\hline Ceiba pentandra (L.) Gaertn. & Shada shimul & $\mathrm{Gr}, \mathrm{Hs}, \mathrm{Rs}$ & Tree, $\mathrm{m} ; \mathrm{pl}$ & Exotic $M A R 2562$ & $\mathrm{Fb}, \mathrm{T}$ \\
\hline Corchorus aestuans $\mathrm{L}$. & Janglipat & $\mathrm{Sj}$ & Shrub; w & Native $S S 442$ & $\mathrm{Fb}, \mathrm{M}$ \\
\hline C. capsularis $\mathrm{L}$. & Pat & $\mathrm{Hg}$ & Herb, er; cv & Native $M A R 2563$ & $\mathrm{Fb}, \mathrm{Vg}$ \\
\hline Durio zibethinus L. + & Durian & $\mathrm{Gr}$ & Tree, $1 ; \mathrm{pl}$ & Exotic MAR 2564 & Fr, M \\
\hline Grewia asiatica $\mathrm{L}$. & Pholsa & Gr, Wl & Tree, s; pl & Native SS 221 & $\mathrm{Fr}$ \\
\hline G. glandulosa Vahl & Pathaka & $\mathrm{Sj}$ & Tree, s; w & Exotic SS 291 & $\mathrm{M}, \mathrm{Fw}$ \\
\hline G. hirsuta Vahl & Kukurbicha & $\mathrm{Rs}, \mathrm{Sj}$ & Shrub; w & Native SS 357 & M \\
\hline G. serrulata DC. & Panisara & $\mathrm{Gr}$ & Tree, s; pl & Native $M A R 2559$ & $\mathrm{M}, \mathrm{Fw}$ \\
\hline G. tiliifolia Vahl & Raktakussum & $\mathrm{Fl}, \mathrm{Sj}$ & Tree, m; w & Native SS 406 & $\mathrm{Fb}, \mathrm{M}$ \\
\hline
\end{tabular}


Table 1 contd.

\begin{tabular}{|c|c|c|c|c|c|}
\hline Scientific name & Bangla name & Habitat & Habit & Origin RSE & Use \\
\hline Hibiscus mutabilis $\mathrm{L}$. & Sthol poddo & $\mathrm{Gr}, \mathrm{Hs}$ & Tree, s; pl & Exotic SSS 2016 & $\mathrm{M}$ \\
\hline H. rosa-sinensis $\mathrm{L}$. & Joba & Gr, Hs, Rs & Shrub; pl & Exotic SSS 2017 & $\mathrm{O}$ \\
\hline H. sabdariffa $\mathrm{L}$. & Chukar & $\mathrm{Gr}, \mathrm{Hs}$ & Shrub; pl & Exotic SSS 2018 & M \\
\hline H. schizopetalus (Dyer) Hook.f. & Jhumko jaba & $\mathrm{Gr}, \mathrm{Hs}$ & Shrub; pl & Exotic SSS 2019 & $\mathrm{O}$ \\
\hline H. surattensis $\mathrm{L}$. & Ram bhindi & $\mathrm{Gr}, \mathrm{Hs}$ & Shrub; pl & Native SSS 2020 & M \\
\hline H. syriacus $\mathrm{L}$. & Sada joba & $\mathrm{Gr}$ & Shrub; pl & Exotic MAR 2565 & $\mathrm{O}$ \\
\hline H. vitifolius $\mathrm{L}$. & Bonkarpas & $\mathrm{Sj}$ & Shrub; w & Native $M A R 2566$ & $\mathrm{M}, \mathrm{O}$ \\
\hline $\begin{array}{l}\text { Malvastrum coromandelianum (L.) } \\
\text { Garcke }\end{array}$ & Clock plant & $\mathrm{Fl}, \mathrm{Sj}$ & Shrub; w & Exotic $M A R 2567$ & M \\
\hline Malvaviscus arboreus Cav. & Morich joba & $\mathrm{Gr}, \mathrm{Hs}$ & Shrub; pl & Exotic $M A R 2568$ & $\mathrm{O}$ \\
\hline Pentapetes phoenicea $\mathrm{L}$. & Bandhuli phul & $\mathrm{Gr}, \mathrm{Hs}$ & Shrub; pl & Native GMH 5012 & $\mathrm{M}, \mathrm{O}$ \\
\hline Pterospermum acerifolium (L.) Willd. & Muchkundo & Gr, Wl & Tree, $1 ; \mathrm{pl}$ & Native GMH 5029 & $\mathrm{M}, \mathrm{T}$ \\
\hline Pterygota alata (Roxb.) R. Br. & Budha narikel & Gr & Tree, $1 ; \mathrm{pl}$ & Native $G M H 5037$ & $\mathrm{M}, \mathrm{T}$ \\
\hline Sida acuta Burm. f. & Kureta & $\mathrm{Fl}, \mathrm{Sj}, \mathrm{Rs}$ & Herb, er; w & Native SS 157 & M \\
\hline S. cordata (Burm. f.) Bross. Waalk. & Pitberela & $\mathrm{Fl}, \mathrm{Sj}, \mathrm{Rs}$ & Herb, er; w & Native SS 104 & M \\
\hline S. cordifolia $\mathrm{L}$. & Shet-berela & $\mathrm{Fl}$, Rs & Herb, er; w & Native $M A R 2569$ & M \\
\hline S. rhombifolia $\mathrm{L}$. & Lal-berela & $\mathrm{Fl}, \mathrm{Rs}$ & Herb, er; w & Native SS 175 & $\mathrm{Fb}, \mathrm{M}$ \\
\hline Thespesia populnea (L.) Sol. ex Corrêa & Porosh pipul & $\mathrm{Gr}, \mathrm{Hs}$ & Tree, s; pl & Native $G M H 5025$ & M \\
\hline Triumfetta rhomboidea Jacq. & Bon okra & $\mathrm{Fl}, \mathrm{Wl}, \mathrm{Sj}$ & Shrub; w & Native SSS 2014 & $\mathrm{Fb}, \mathrm{M}$ \\
\hline Urena lobata $\mathrm{L}$. & Atlera & $\mathrm{Fl}, \mathrm{Sj}, \mathrm{Rs}$ & Shrub; w & Native SS 115 & $\mathrm{Fb}, \mathrm{M}$ \\
\hline \multicolumn{6}{|l|}{ Lecythidaceae A. Rich. } \\
\hline Barringtonia acutangula (L.) Gaertn. & Hijal & $\mathrm{Ml}, \mathrm{Sj}, \mathrm{Wtl}$ & Tree, m; pl-w & Native GMH 5029 & M. O \\
\hline Careya arborea Roxb. & Kumbi & Wl & Tree, $1 ; \mathrm{w}$ & Native $M A R 77$ & M \\
\hline C. herbacea Roxb.\# & Bhuidalim & $\mathrm{Sj}, \mathrm{Wl}$ & Herb, er; w & Native SS 230 & M \\
\hline Couroupita guianensis Aubl. & Naglingom & Gr, Wl & Tree, $1 ; \mathrm{pl}$ & Exotic GMH 5022 & $\mathrm{M}, \mathrm{Pf}$ \\
\hline Gustavia superba (Kunth) O. Berg + & Gustav & Gr, Wl & Tree, $1 ; \mathrm{pl}$ & Exotic MAR 2717 & $\mathrm{O}$ \\
\hline Bixaceae Kunth & & & & & \\
\hline Bixa orellana $\mathrm{L}$. & Belatihaldi & Gr & Tree, s; pl & Exotic MAR 2570 & Dy, M \\
\hline \multicolumn{6}{|l|}{ Passifloraceae Juss. ex Roussel } \\
\hline Passiflora coccinea Aubl. & Jhumko phul & $\mathrm{Gr}, \mathrm{Hs}$ & Herb, vi; pl & Exotic SSS 2021 & $\mathrm{M}, \mathrm{O}$ \\
\hline P. edulis Sims & Passion phal & $\mathrm{Gr}, \mathrm{Hs}$ & Herb, vi; pl & Exotic SSS 2022 & Fr, M \\
\hline P. foetida $\mathrm{L}$. & Jhumka lata & $\mathrm{Sj}$ & Herb, vi; w & Exotic SS 227 & Fr, M \\
\hline \multicolumn{6}{|l|}{ Caricaceae Dumort. } \\
\hline \multicolumn{6}{|l|}{ Cucurbitaceae Juss. } \\
\hline Benincasa hispida (Thunb.) Cogn. & Chalkumra & Hs & Herb, vi; cv & Exotic MAR 2572 & $\mathrm{Vg}$ \\
\hline $\begin{array}{l}\text { Citrullus lanatus (Thunb.) Matsum. \& } \\
\text { Nakai }\end{array}$ & Tormuj & Hs & Herb, vi; cv-w & Exotic MAR 2573 & Fr \\
\hline Coccinia grandis $(\mathrm{L}$.) Voigt & Telakucha & $\mathrm{Bw}^{1}, \mathrm{Sj}, \mathrm{Wl}$ & Herb, vi; w & Native SS 445 & $\mathrm{M}, \mathrm{Vg}$ \\
\hline Cucumis melo $\mathrm{L}$. & Kakur & $\mathrm{Fl}, \mathrm{Hs}$ & Herb, vi; cv-w & Exotic $M A R 2574$ & Fr, $\mathrm{Vg}$ \\
\hline C. sativus $\mathrm{L}$. & Khira & $\mathrm{Fl}, \mathrm{Hs}$ & Herb, vi; cv-w & Native $M A R 2575$ & $\mathrm{Vg}$ \\
\hline Cucurbita maxima Duchesne & Kumra & $\mathrm{Fl}, \mathrm{Hs}$ & Herb, vi; cv-w & Exotic MAR 2576 & $\mathrm{Vg}$ \\
\hline Diplocyclos palmatus (L.) C. Jeffrey & Mama kola & $\mathrm{Fl}, \mathrm{Sj}$ & Herb, vi; w & Native SS 444 & M \\
\hline Lagenaria siceraria (Molina) Standl. & Lao & $\mathrm{Fl}, \mathrm{Hs}$ & Herb, vi; cv-w & Exotic MAR 2577 & $\mathrm{Vg}$ \\
\hline Luffa acutangula (L.) Roxb. & Jhinga & $\mathrm{Fl}, \mathrm{Hs}$ & Herb, vi; cv-w & Native $M A R 2578$ & $\mathrm{Vg}$ \\
\hline
\end{tabular}


Table 1 contd.

\begin{tabular}{|c|c|c|c|c|c|}
\hline Scientific name & Bangla name & Habitat & Habit & Origin RSE & Use \\
\hline L. cylindrica (L.) M. Roem. & Dhundal & $\mathrm{Fl}, \mathrm{Hs}$ & Herb, vi; cv-w & Native $M A R 2579$ & $\mathrm{Vg}$ \\
\hline Momordica charantia L. & Korolla & $\mathrm{Fl}, \mathrm{Hs}$ & Herb, vi; cv-w & Native $M A R 2580$ & $\mathrm{Vg}$ \\
\hline M. dioica Roxb. ex Willd. & Kakrol & $\mathrm{Fl}, \mathrm{Hs}, \mathrm{Sj}$ & Herb, vi; cv-w & Native $M A R 2581$ & $\mathrm{Vg}$ \\
\hline Mukia maderaspatana (L.) M. Roem. & Agmukhi & $\mathrm{Bw}^{1}, \mathrm{Gl}, \mathrm{Sj}$ & Herb, vi; w & Native SS 189 & M \\
\hline Solena amplexicaulis (Lam.) Gandhi & Rakhal sosha & $\mathrm{Sj}$ & Herb, vi; w & Native $G M H 5032$ & M \\
\hline Trichosanthes dioica Roxb. & Potol & Hs & Herb, vi; cv & Native $G M H 5047$ & $\mathrm{Vg}$ \\
\hline T. costata Blume & Banpatol & $\mathrm{Sj}$ & Herb, vi; w & Native SS 376 & M \\
\hline T. cucumerina $\mathrm{L}$. & Chichinga & $\mathrm{Fl}, \mathrm{Hs}$ & Herb, vi; cv & Native $M A R 2715$ & $\mathrm{Vg}$ \\
\hline T. tricuspidata Lour. & Makal & $\mathrm{Fl}, \mathrm{Hs}$ & Herb, vi; cv-w & Exotic GMH 5003 & M \\
\hline \multicolumn{6}{|l|}{ Salicaceae Mirb. } \\
\hline Flacourtia indica (Burm. f.) Merr. & Bauchi & $\mathrm{Bw}^{1}, \mathrm{Sj}$ & Shrub; w & Native SS 200 & Fr, M \\
\hline \multicolumn{6}{|l|}{ Capparaceae Juss. } \\
\hline Capparis zeylanica $\mathrm{L}$. & Katai & $\mathrm{Sj}$ & Shrub; w & Native SSS 2023 & Fr, M \\
\hline Crateva nurvala Buch.-Ham. & Borun & Wtl & Tree, s; w & Native GMH 5039 & Fw, M \\
\hline \multicolumn{6}{|l|}{ Cleomaceae Bercht. \& J. Presl } \\
\hline Cleome houtteana Schltdl. & Hurhuria & $\mathrm{Gr}, \mathrm{Rs}, \mathrm{Fl}$ & Herb, er; pl-w & Exotic SSS 2026 & $\mathrm{O}$ \\
\hline C. rutidosperma DC. & Nil hurhurey & $\mathrm{Bw}^{1}, \mathrm{Fl}, \mathrm{Gl}, \mathrm{Rs}$ & Herb, er; w & Exotic SSS 2027 & M \\
\hline C. viscosa $\mathrm{L}$. & Halud hurhurey & $\mathrm{Bw}^{1}, \mathrm{Fl}, \mathrm{Gl}, \mathrm{Rs}$ & Herb, er; w & Native GMH 5057 & M \\
\hline \multicolumn{6}{|l|}{ Brassicaceae Burnett } \\
\hline Brassica juncea (L.) Czern. & Sarisha & $\mathrm{Gr}, \mathrm{Hs}$ & Herb, er; cv & Exotic MAR 2581 & Oy, Vg \\
\hline Cardamine cf. flexuosa With. & Unknown & $\mathrm{Fl}, \mathrm{Gr}$ & Herb, er; w & Exotic MAR 101 & M \\
\hline Rorippa indica (L.) Hiern & Bansarisha & $\mathrm{Fl}, \mathrm{Gr}$ & Herb, er; w & Native SSS 2024 & M \\
\hline \multicolumn{6}{|l|}{ Moringaceae Martinov } \\
\hline Moringa oleifera Lamk. & Shajna & Hs, Rs & Tree, $\mathrm{m} ; \mathrm{pl}$ & Exotic MAR 168 & $\mathrm{M}, \mathrm{Vg}$ \\
\hline \multicolumn{6}{|l|}{ Sapotaceae Juss. } \\
\hline Chrysophyllum cainito $\mathrm{L}$. & Star apple & Gr & Tree, $\mathrm{m} ; \mathrm{pl}$ & Exotic $M A R 2582$ & $\mathrm{Fr}, \mathrm{M}$ \\
\hline $\begin{array}{l}\text { Madhuca longifolia (J. König ex L.) J.F. } \\
\text { Macbr. }\end{array}$ & Mohua & Gr, Rs & Tree, m; pl-w & Native SS 436 & $\mathrm{M}, \mathrm{Oy}$ \\
\hline Manilkara zapota (L.) P. Royen & Sopheda & $\mathrm{Gr}, \mathrm{Hs}$ & Tree, $\mathrm{m} ; \mathrm{pl}$ & Exotic GMH 5042 & Fr, M \\
\hline Mimusops elengi $\mathrm{L}$. & Bokul & $\mathrm{Gr}, \mathrm{Hs}$ & Tree, m; pl-w & Native $G M H 5035$ & $\mathrm{M}, \mathrm{O}$ \\
\hline \multicolumn{6}{|l|}{ Ebenaceae Gürke } \\
\hline Diospyros discolor Willd. & Bilati gab & $\mathrm{Gr}, \mathrm{Hs}$ & Tree, $\mathrm{m} ; \mathrm{pl}$ & Exotic SSS 2025 & Fr, M \\
\hline D. malabarica (Desr.) Kostel. & Deshi gab & Wl & Tree, $\mathrm{m} ; \mathrm{w}$ & Native MAR 169 & Fr, M \\
\hline D. montana Roxb. & Tamal & Gr, Wl & Tree, s; pl-w & Native MAR 105 & M, Po \\
\hline Myrsinaceae R. Br. & & & & & \\
\hline Ardisia solanacea (Poir.) Roxb. & Banjam & Wl & Shrub; w & Native SS 264 & $\mathrm{M}, \mathrm{O}$ \\
\hline Hydrangeaceae Dumort. & & & & & \\
\hline Hydrangea macrophylla (Thunb.) Ser. + & Hydrangea & Gr & Shrub; pl & Exotic MAR 2583 & M \\
\hline Crassulaceae J. St.-Hil. & & & & & \\
\hline Kalachoe pinnata (Lam.) Pers. & Patharkuchi & $\mathrm{Gr}, \mathrm{Hs}$ & Herb, er; pl & Exotic MAR 2714 & $\mathrm{M}, \mathrm{O}$ \\
\hline Rosaceae Juss. & & & & & \\
\hline Eriobotrya japonica (Thunb.) Lindl. + & Lokat & Gr & Tree, s; pl & Exotic MAR 2655 & M \\
\hline$*$ Rosa $\times$ centifolia $\mathrm{L}$. & Golap & $\mathrm{Gr}, \mathrm{Hs}$ & Shrub; cv & Exotic $M A R 2656$ & $\mathrm{M}, \mathrm{O}$ \\
\hline R. chinensis Jacq. & Jangli golap & Gr, Hs, Ml & Shrub; pl-w & Exotic MAR 2657 & Fn \\
\hline Rubus armeniacus Focke + & Blackberry & Gr, Hs & Shrub; pl & Exotic GMH 5081 & Fr \\
\hline
\end{tabular}


Table 1 contd.

\begin{tabular}{|c|c|c|c|c|c|}
\hline Scientific name & Bangla name & Habitat & Habit & Origin RSE & Use \\
\hline $\begin{array}{l}\text { *Fragaria } \times \text { ananassa } \text { (Duchesne ex } \\
\text { Weston) Duchesne ex Rozier }+\end{array}$ & Stawberry & Gr. Hs & Herb, cr; cv & Exotic GMH 5091 & $\mathrm{Fr}$ \\
\hline F. vesca L. + & Jangli strawberry & $\mathrm{Sj}$ & Herb, cr; w & Exotic GMH 5004 & M \\
\hline \multicolumn{6}{|l|}{ Mimosaceae R. Br. } \\
\hline Acacia auriculiformis Benth. & Akashmoni & $\mathrm{Bw}^{2}, \mathrm{Fl}, \mathrm{Rs}, \mathrm{Wl}$ & Tree, 1; pl-w & Exotic $S S 450$ & $\mathrm{~T}$ \\
\hline A. catechuoides (Roxb.) Benth. & Khoir & $\mathrm{Gr}$ & Tree, m; pl & Native $M A R 2584$ & M, Tn \\
\hline A. concinna (Willd.) DC. ++ & Bon babla & $\mathrm{Sj}, \mathrm{Wl}$ & Shrub, sc; w & Native $M A R 2585$ & $\mathrm{Co}, \mathrm{M}$ \\
\hline A. farnesiana (L.) Willd. & Bilatibabla & $\mathrm{W} 1$ & Tree, m; pl & Exotic SS 451 & Fw, M \\
\hline A. mangium Willd. & Mangium & Gr, Wl & Tree, $1 ; \mathrm{pl}$ & Exotic $S S 452$ & $\mathrm{~T}$ \\
\hline A. nilotica (L.) Delile & Babla & $\mathrm{Fl}, \mathrm{Rs}$ & Tree, m; w & Native SSS 2028 & $\mathrm{Gu}, \mathrm{M}$ \\
\hline Adenanthera pavonina $\mathrm{L}$. & Rakta chandan & $\mathrm{Gr}$ & Tree, m; pl-w & Native $M A R 2586$ & $\mathrm{M}, \mathrm{O}$ \\
\hline Albizia lebbeck (L.) Benth. & Kalo koroi & Gr, Rs, Wl & Tree, 1; pl-w & Native SSS 2029 & $\mathrm{~T}$ \\
\hline A. lucidior (Steud.) I.C. Nielsen & Motor koroi & Wl & Tree, l; w & Native SSS 2030 & $\mathrm{~T}$ \\
\hline A. procera (Roxb.) Benth. & Shada/sil koroi & $\mathrm{Bw}^{2}, \mathrm{Gr}, \mathrm{Rs}$ & Tree, 1; pl-w & Native SSS 2031 & $\mathrm{~T}$ \\
\hline A. richardiana (Voigt) King \& Prain & Raj siris & Gr, Rs & Tree, 1; pl-w & Exotic SSS 2032 & $\mathrm{M}, \mathrm{T}$ \\
\hline A. saman (Jacq.) Merr. & Shirish & Rs, Wl, Ml & Tree, 1; pl-w & Exotic SSS 2033 & $\mathrm{~T}, \mathrm{Sd}$ \\
\hline Calliandra haematocephala Hassk. & Golapi callandra & Gr, Rs & Tree, s; pl & Exotic SSS 2034 & $\mathrm{O}$ \\
\hline Leucaena leucocephala (Lam.) de Wit & Ipil-ipil & $\mathrm{Fl}, \mathrm{Rs}, \mathrm{Wl}$ & Tree, 1; pl-w & Exotic SSS 2035 & Fw, T \\
\hline Mimosa diplotricha Sauvalle & Baralajjaboti & $\mathrm{Sj}, \mathrm{Rs}$ & Shrub; w & Exotic SS 393 & M \\
\hline M. pudica $\mathrm{L}$. & Lajjaboti & $\mathrm{Gl}, \mathrm{Fl}, \mathrm{Rs}$ & Herb, pr; w & Exotic SS 110 & M \\
\hline \multicolumn{6}{|l|}{ Caesalpiniaceae R. Br. } \\
\hline Bauhinia acuminata $\mathrm{L}$. & Sadakanchon & Gr, Rs & Tree, s; pl-w & Native $S S 219$ & $\mathrm{M}, \mathrm{O}$ \\
\hline B. malabarica Roxb. & Kanchon & Gr, Rs & Tree, m; pl & Native $M A R 2587$ & $\mathrm{M}, \mathrm{O}$ \\
\hline B. purpurea $\mathrm{L}$. & Rakto kanchon & Gr, Rs & Tree, m; pl & Native $M A R 2588$ & $\mathrm{M}, \mathrm{O}$ \\
\hline B. variegata $\mathrm{L}$. & Lal kanchon & Gr, Rs & Tree, s; pl & Native $M A R 2589$ & $\mathrm{M}, \mathrm{O}$ \\
\hline Brownea coccinea Jacq. & Pakhi phul & Gr & Tree, s; pl & Exotic $M A R 2590$ & $\mathrm{M}, \mathrm{O}$ \\
\hline Caesalpinia digyna Rottler & Amalkuchi & $\mathrm{Sj}$ & Shrub, sc; w & Native SS 369 & M \\
\hline Cassia fistula $\mathrm{L}$. & Badarlathi & Gr, Rs & Tree, $\mathrm{m} ; \quad \mathrm{pl}-\mathrm{w}$ & Native $S S 453$ & $\mathrm{M}, \mathrm{O}$ \\
\hline C. javanica $\mathrm{L} .++$ & Bon sonalu & Gr, Rs & Tree, m; pl & Native $S S 454$ & $\mathrm{O}$ \\
\hline C. renigera Benth. + & $\begin{array}{l}\text { Burmese pink } \\
\text { shonalu }\end{array}$ & Gr, Rs & Tree, m; pl & Exotic MAR 2591 & $\mathrm{O}$ \\
\hline $\begin{array}{l}\text { C. javanica subsp. nodosa (Roxb.) K. } \\
\text { Larsen \& S.S. Larsen }+\end{array}$ & Burmese shonalu & Gr, Rs & Tree, m; pl & Exotic $M A R 2592$ & $\mathrm{O}$ \\
\hline Delonix regia (Hook.) Raf. & Krishnachura & Rs & Tree, 1; pl-w & Exotic GMH 5067 & $\mathrm{O}$ \\
\hline $\begin{array}{l}\text { Peltophorum pterocarpum (DC.) K. } \\
\text { Heyne }\end{array}$ & Radha chura & Gr, Rs & Tree, 1; pl-w & Exotic GMH 5049 & $\mathrm{O}$ \\
\hline Saraca asoca (Roxb.) Willd. & Ashok & Gr, Wl & Tree, $\mathrm{m} ; \quad \mathrm{pl}-\mathrm{w}$ & Native $S S 370$ & $\mathrm{M}, \mathrm{O}$ \\
\hline S. indica $\mathrm{L}$. & Panshi ashok & Gr, Wl & Tree, $\mathrm{m} ; \quad \mathrm{pl}-\mathrm{w}$ & Exotic SSS 2036 & $\mathrm{M}, \mathrm{O}$ \\
\hline Senna alata (L.) Roxb. & Dadmardan & $\mathrm{Fl}, \mathrm{Hs}, \mathrm{Rs}$ & Shrub; w & Exotic $S S 455$ & M \\
\hline S. occidentalis (L.) Link & Barakalkesunda & $\mathrm{Fl}, \mathrm{Rs}$ & Shrub; w & Exotic SS 146 & M \\
\hline S. siamea (Lam.) H.S. Irwin \& Barn. & Minjiri & $\mathrm{Fl}, \mathrm{Gr}, \mathrm{Wl}$ & Tree, 1; pl-w & Exotic SS 456 & Fw, O \\
\hline S. sophera (L.) Roxb. & Kalkeshunda & $\mathrm{Fl}, \mathrm{Sj}, \mathrm{Rs}$ & Shrub; w & Exotic SS 372 & M \\
\hline S. tora (L.) Roxb. & Araj & $\mathrm{Fl}, \mathrm{Gl}, \mathrm{Rs}$ & Herb, er; w & Exotic SS 139 & M \\
\hline Tamarindus indica $\mathrm{L}$. & Tetul & Gr, Hs, Wl & Tree, 1; pl-w & Exotic $M A R 2593$ & $\mathrm{Fr}, \mathrm{T}$ \\
\hline Xylia xylocarpa (Roxb.) Taub. & Lohakath & Gr, Wl & Tree, $1 ; \mathrm{pl}$ & Native SSS 2037 & $\mathrm{~T}$ \\
\hline
\end{tabular}


Table 1 contd.

\begin{tabular}{|c|c|c|c|c|c|}
\hline Scientific name & Bangla name & Habitat & Habit & Origin RSE & Use \\
\hline \multicolumn{6}{|l|}{ Fabaceae Lindl. } \\
\hline Abrus precatorius $\mathrm{L}$. & Kunch & $\mathrm{Sj}$ & Liana; w & Native MAR 171 & M \\
\hline Aeschynomene americana $\mathrm{L}$. & Shola & $\mathrm{Fl}, \mathrm{Wtl}$ & Herb, er; w & Exotic MAR 384 & $\mathrm{Fd}, \mathrm{Fu}$ \\
\hline A. indica $\mathrm{L}$. & Bhatshola & $\mathrm{Fl}, \mathrm{Wtl}$ & Herb, er; w & Native SSS 2038 & $\mathrm{Gm}, \mathrm{M}$ \\
\hline Alysicarpus vaginalis DC. & Pinnata & Gl & Herb, er; w & Native MAR 136 & $\mathrm{Fd}, \mathrm{Gm}$ \\
\hline Butea monosperma (Lam.) Taub. & Palash & Gr, Rs, Wl & Tree, m; pl-w & Native $M A R 76$ & Dy, M \\
\hline Cajanus cajan (L.) Millsp. & Arhar & $\mathrm{Fl}, \mathrm{Hs}$ & Shrub; pl-w & Exotic SSS 2039 & $\mathrm{M}, \mathrm{Pu}$ \\
\hline C. scarabaeoides (L.) Thouars & Banurkalki & $\mathrm{Sj}$ & Herb, tw; w & Native MAR 170 & M \\
\hline Canavalia gladiata (Jacq.) DC. & Moushim & Hs, Wl & Liana; pl & Native SSS 2040 & $\mathrm{M}, \mathrm{Vg}$ \\
\hline Centrosema pubescens Benth. & Ban aparajita & $\mathrm{Sj}$ & Herb, tw; w & Exotic SS 247 & Fo, Gm \\
\hline Clitoria ternatea $\mathrm{L}$. & Aparajita & $\mathrm{Gr}, \mathrm{Hs}$ & Herb, tw; pl-w & Exotic SSS 2041 & $\mathrm{M}, \mathrm{O}$ \\
\hline $\begin{array}{l}\text { Codoriocalyx gyroides (Roxb. ex Link) } \\
\text { Hassk. }\end{array}$ & Codaridis & $\mathrm{Sj}$ & Shrub; w & Native $S S 383$ & M \\
\hline Crotalaria calycina Schrank. & Kali jhunjhuni & $\mathrm{Fl}, \mathrm{Rs}$ & Herb, er; w & Native $S S 168$ & M \\
\hline C. pallida Aiton & Jhunjhuni & $\mathrm{Fl}, \mathrm{Rs}$ & Herb, er; w & Native $S S 381$ & $\mathrm{Fb}, \mathrm{M}$ \\
\hline C. prostrata Rottler ex Willd. & Shyaon jhunjhuni & $\mathrm{Fl}, \mathrm{Gl}$ & Herb, pr; w & Native SS 164 & M \\
\hline C. sessiliflora $\mathrm{L}$. & Silai jhunjhuni & Gl & Herb, er; w & Native $S S 382$ & M \\
\hline Dalbergia sissoo DC. & Sisoo & Gr, Wl, Rs & Tree, $1 ;$ pl-w & Native SSS 2042 & $\mathrm{~T}$ \\
\hline D. stipulacea Roxb. ++ & Dadbari & $\mathrm{Sj}, \mathrm{Wl}$ & Shrub, sc; w & Native SSS 2043 & Fw, M \\
\hline D. volubilis Roxb. ++ & Angi lata & $\mathrm{Sj}$ & Shrub, sc; w & Native SSS 2044 & M, Po \\
\hline Derris scandens (Roxb.) Benth. & Kalia lata & $\mathrm{Sj}, \mathrm{Wl}$ & Liana; w & Native $S S 457$ & $\mathrm{Fb}, \mathrm{M}$ \\
\hline Desmodium gangeticum (L.) DC. & Salpani & $\mathrm{Fl}, \mathrm{Sj}, \mathrm{Wl}$ & Shrub; w & Native $M A R 138$ & M \\
\hline D. heterophyllum (Willd.) DC. & Bonmotorshuti & $\mathrm{Fl}, \mathrm{Gl}$ & Herb, pr; w & Native SS 384 & $\mathrm{Fd}, \mathrm{M}$ \\
\hline Erythrina fusca Lour. & Kantamandar & Ml, Rs & Tree, s; pl-w & Native SSS 2045 & Fn, M \\
\hline E. stricta Roxb. & Raktamandar & Ml, Rs & Tree, s; pl-w & Native SSS 2046 & Fn, M \\
\hline E. variegata $\mathrm{L}$. & Parijat & $\mathrm{Fl}, \mathrm{Ml}, \mathrm{Rs}$ & Tree, s; pl-w & Native SSS 2047 & Fn, M \\
\hline $\begin{array}{l}\text { Flemingia macrophylla (Willd.) Kuntze } \\
\text { ex Merr. }\end{array}$ & Barasalpan & $\mathrm{Sj}, \mathrm{Wl}$ & Shrub; w & Native SS 249 & $\mathrm{M}, \mathrm{Mu}$ \\
\hline F. stricta Roxb. ex Aiton & Charchara & $\mathrm{Fl}, \mathrm{Gl}$ & Shrub; w & Native $S S 386$ & M \\
\hline Gliricidia sepium (Jacq.) Walp. + & Basantamanjuri & Ml, Rs & Tree, $\mathrm{m} ; \mathrm{pl}$ & Exotic SS 387 & $\mathrm{Fd}, \mathrm{Gm}$ \\
\hline $\begin{array}{l}\text { Grona heterocarpos (L.) H. Ohashi \& K. } \\
\text { Ohashi }\end{array}$ & Kodalia & $\mathrm{Sj}, \mathrm{Wl}$ & Shrub; w & Native SS 387 & $\mathrm{Fd}, \mathrm{Gm}$ \\
\hline G. triflora (L.) H. Ohashi \& K. Ohashi & Kulalia & $\mathrm{Fl}, \mathrm{Gl}$ & Herb, pr; w & Native SS 385 & $\mathrm{M}, \mathrm{Sb}$ \\
\hline Indigofera tinctoria $\mathrm{L} .++$ & Nil & $\mathrm{Fl}, \mathrm{Gl}$ & Shrub; w & Native $S S 138$ & Dy, M \\
\hline Medicago polymorpha $\mathrm{L}$. & Medla & $\mathrm{Fl}, \mathrm{Gl}$ & Herb, pr; w & Exotic SSS 2048 & Fo, Gm \\
\hline Millettia peguensis Ali ++ & Tuma & Gr, Rs & Tree, $1 ; \mathrm{pl}$ & Native SSS 2049 & $\mathrm{O}, \mathrm{T}$ \\
\hline Mucuna pruriens (L.) DC. & Bilaichimti & $\mathrm{Sj}, \mathrm{Wl}$ & Liana; w & Native $S S 252$ & M, Po \\
\hline Phyllodium pulchellum (L.) Desv. & Jatsalpani & Wl & Shrub; w & Native MAR 137 & M \\
\hline Pongamia pinnata (L.) Pierre & Karach & Gr & Tree, m; pl-w & Native SSS 2050 & $\mathrm{Fu}, \mathrm{M}$ \\
\hline Pterocarpus indicus Willd. ++ & Padauk & Gr & Tree, 1 ; pl-w & Native SSS 2051 & $\mathrm{M}, \mathrm{O}$ \\
\hline Pueraria phaseoloides (Roxb.) Benth. & Mugi kunch & $\mathrm{Fl}, \mathrm{Sj}$ & Liana; w & Native SSS 2052 & $\mathrm{Fd}$ \\
\hline Sesbania cannabina (Retz.) Pers. & Dhonchi & $\mathrm{Fl}, \mathrm{Hs}, \mathrm{Ml}$ & Shrub; cv-w & Native SSS 2053 & $\mathrm{Fb}, \mathrm{Gm}$ \\
\hline S. grandiflora (L.) Pers. & Bak phul & $\mathrm{Gr}, \mathrm{Hs}, \mathrm{Ml}$ & Tree, s; pl & Exotic SSS 2054 & $\mathrm{Fd}, \mathrm{Vg}$ \\
\hline Spatholobus parviflorus (DC.) Kuntze & Polasia lata & $\mathrm{Sj}, \mathrm{Wl}$ & Liana; w & Native SSS 2055 & $\mathrm{Fb}, \mathrm{M}$ \\
\hline Tephrosia candida (Roxb.) DC. & Bilakshani & $\mathrm{Fl}, \mathrm{Sj}$ & Shrub; w & Native SSS 2056 & $\mathrm{Gm}, \mathrm{M}$ \\
\hline T. purpurea (L.) Pers. & Bannil & $\mathrm{Fl}, \mathrm{Sj}$ & Shrub; w & Native MAR 139 & $\mathrm{Gm}, \mathrm{M}$ \\
\hline
\end{tabular}


Table 1 contd.

\begin{tabular}{|c|c|c|c|c|c|}
\hline Scientific name & Bangla name & Habitat & Habit & Origin RSE & Use \\
\hline Uraria lagopodioides (L.) DC. & Chakulia & $\mathrm{Sj}, \mathrm{Wl}$ & Herb; w & Native $S S 388$ & $\mathrm{M}$ \\
\hline Vicia hirsuta (L.) Gray & Masurchana & Gl & Herb, pr; w & Native $S S 458$ & $\mathrm{Fd}, \mathrm{M}$ \\
\hline \multicolumn{6}{|l|}{ Proteaceae Juss. } \\
\hline Grevillea robusta A. Cunn. ex R. Br. & Silver oak & $\mathrm{Gr}$ & Tree, $1 ; \mathrm{pl}$ & Exotic GMH 5077 & Fw, O \\
\hline \multicolumn{6}{|l|}{ Haloragaceae R. Br. } \\
\hline Myriophyllum tuberculatum Roxb. & Kulabahupatri & Wtl & Herb, sm; w & Native $M A R 141$ & M \\
\hline \multicolumn{6}{|l|}{ Lythraceae J. St.-Hil. } \\
\hline Ammannia multiflora Roxb. & Acidpatta & $\mathrm{Fl}, \mathrm{Wtl}$ & Herb, er; w & Native $G M H 5087$ & M \\
\hline Cuphea hyssopifolia Kunth & Panica & Gr & Herb, er; pl & Exotic $G M H 5183$ & $\mathrm{O}$ \\
\hline Lagerstroemia indica $\mathrm{L}$. & Jarul & Gr, Rs & Tree, s; pl & Native GMH 5052 & $\mathrm{O}, \mathrm{T}$ \\
\hline L. parviflora Roxb. & Sidha jarul & $\mathrm{Gr}, \mathrm{Rs}$ & Tree, s; Pl & Native GMH 5059 & $\mathrm{O}, \mathrm{Fw}$ \\
\hline L. speciosa (L.) Pers. & Jarul & Gr, Ml, Rs & Tree, 1; pl-w & Native SS 459 & $\mathrm{O}$ \\
\hline Lawsonia inermis $\mathrm{L}$. & Mehedi & $\mathrm{Gr}, \mathrm{Hs}$ & Tree, s; pl & Exotic GMH 5045 & Dy, M \\
\hline Punica granatum $\mathrm{L}$. & Dalim & Hs & Shrub; pl & Exotic GMH 5087 & Dy, Fr \\
\hline Rotala indica (Willd.) Koehne & Ghurni & $\mathrm{Fl}, \mathrm{Ml}$ & Herb, er; w & Native $G M H 5013$ & M \\
\hline $\begin{array}{l}\text { R. rotundifolia (Buch.-Ham. ex Roxb.) } \\
\text { Koehne }\end{array}$ & Dim ghurni & $\mathrm{Fl}, \mathrm{Ml}$ & Herb, cr; w & Native GMH 5001 & M \\
\hline Trapa incisa Siebold \& Zucc. & Paniphal & Wtl & Herb, fr, w & Native GMH 5031 & Ed, M \\
\hline \multicolumn{6}{|l|}{ Thymelaeaceae Juss. } \\
\hline Aquilaria malaccensis Lam. \# & Agar & Gr, Rs & Tree, $1 ; \mathrm{pl}$ & Native $G M H 5023$ & $\mathrm{M}, \mathrm{Pf}$ \\
\hline \multicolumn{6}{|l|}{ Myrtaceae Juss. } \\
\hline Callistemon citrinus (Curtis) Skeels & Bottlebrush & Gr, Rs, Hs & Tree, s; pl & Exotic GMH 5062 & $\mathrm{O}$ \\
\hline $\begin{array}{l}\text { Corymbia citriodora (Hook.) K.D. Hill } \\
\& \text { L.A.S. Johnson }\end{array}$ & Eucalyptus & Gr, Rs & Tree, $1 ; \mathrm{pl}$ & Exotic MAR 2594 & $\mathrm{Co}, \mathrm{M}$ \\
\hline Eucalyptus camaldulensis Dehnh. & Eucalyptus & Gr, Rs & Tree, $1 ; \mathrm{pl}$ & Exotic GMH 5003 & $\mathrm{~T}, \mathrm{O}$ \\
\hline Myrcia bracteata (Rich.) DC. & Hijlimendi & $\mathrm{Sj}, \mathrm{Wl}$ & Tree, m; w & Exotic SS 394 & Ed, M \\
\hline Psidium guajava $\mathrm{L}$. & Peyara & $\mathrm{Bw}^{2}, \mathrm{Gr}, \mathrm{Hs}$ & Tree, $\mathrm{s} ; \quad \mathrm{pl}-\mathrm{w}$ & Exotic SS 460 & Fr, M \\
\hline $\begin{array}{l}\text { Syzygium aromaticum (L.) Merr. \& L.M. } \\
\text { Perry }\end{array}$ & Labongo & Gr & Tree, $1 ; \mathrm{pl}$ & Exotic $M A R 2816$ & $\mathrm{M}, \mathrm{Sp}$ \\
\hline S. cumini (L.) Skeels & Kalojam & Rs, Wl, Hs & Tree, $1 ;$ pl-w & Native $S S 461$ & Fr, $\mathrm{T}$ \\
\hline S. fruticosum DC. & Khudi jam & $\mathrm{Bw}^{2}, \mathrm{Sj}, \mathrm{Wl}$ & Tree, $\mathrm{m} ; \mathrm{w}$ & Native SS 266 & Fr, M \\
\hline S. grande (Wight) Walp. & Dhaki jam & Wl & Tree, $1 ; \mathrm{pl}$ & Native $S S 176$ & $\mathrm{~T}$ \\
\hline S. jambos (L.) Alston & Golapjam & Gr & Tree, $1 ; \mathrm{pl}$ & Native GMH 5033 & Fr, M \\
\hline S. nervosum A.Cunn. ex DC. & Botijam & $\mathrm{Sj}$ & Tree, s; w & Native $G M H 5055$ & M \\
\hline $\begin{array}{l}\text { S. samarangense (Blume) Merr. \& L.M. } \\
\text { Perry }\end{array}$ & Jamrul & $\mathrm{Gr}, \mathrm{Hs}$ & Tree, $1 ; \mathrm{pl}$ & Native $M A R 2595$ & Fr \\
\hline \multicolumn{6}{|l|}{ Onagraceae Juss. } \\
\hline Ludwigia adscendens (L.) H. Hara & Keshordam & Wtl & Herb, fr; w & Native SS 462 & M \\
\hline L. hyssopifolia (G. Don) Exell & Panipalong & $\begin{array}{l}\mathrm{Bw}^{1}, \mathrm{Fl}, \mathrm{Ml}, \\
\mathrm{Wtl}\end{array}$ & Herb, er; w & Exotic $S S 463$ & M \\
\hline L. perennis $\mathrm{L}$. & Amorkura & $\mathrm{Fl}, \mathrm{Gl}$ & Herb, er; w & Native $S S 465$ & M \\
\hline \multicolumn{6}{|l|}{ Melastomataceae Juss. } \\
\hline Melastoma malabathricum $\mathrm{L}$. & Ban tejpata & $\mathrm{Fl}, \mathrm{Sj}, \mathrm{Wl}$ & Shrub; w & Native SS 166 & $\mathrm{M}$ \\
\hline \multicolumn{6}{|l|}{ Combretaceae R. Br. } \\
\hline Combretum grandiflorum G. Don & Brush phul & $\mathrm{Gr}, \mathrm{Hs}$ & Liana; pl & Exotic $M A R 2596$ & $\mathrm{M}, \mathrm{O}$ \\
\hline C. indicum (L.) De Filipps & Madobi lata & $\mathrm{Gr}, \mathrm{Hs}$ & Liana; pl & Native $M A R 2597$ & $\mathrm{M}, \mathrm{O}$ \\
\hline
\end{tabular}


Table 1 contd.

\begin{tabular}{|c|c|c|c|c|c|}
\hline Scientific name & Bangla name & Habitat & Habit & Origin RSE & Use \\
\hline $\begin{array}{l}\text { Terminalia arjuna (Roxb. ex DC.) Wight } \\
\& \text { Arn. }\end{array}$ & Arjun & Gr, Rs & Tree, 1; pl-w & Native $S S 466$ & $\mathrm{M}$ \\
\hline T. bellirica (Gaertn.) Roxb. & Bohera & Gr, Rs, Wl & Tree, 1; pl-w & Native SS 467 & M \\
\hline T. catappa $\mathrm{L}$. & Kathbadam & $\mathrm{Gr}, \mathrm{Rs}$ & Tree, l; pl-w & Native SS 468 & Ed, M \\
\hline T. chebula Retz. & Horitoki & Gr, Rs, Wl & Tree, 1; pl-w & Native SS 469 & M \\
\hline \multicolumn{6}{|l|}{ Cornaceae Bercht. ex J. Presl } \\
\hline Alangium salviifolium (L.f.) Wangerin & Ankura & $\mathrm{Gr}, \mathrm{Sj}$ & Tree, $\mathrm{m}, \mathrm{pl}$ & Native $M A R 2718$ & $\mathrm{M}, \mathrm{Fu}$ \\
\hline \multicolumn{6}{|l|}{ Olacaceae R. Br. } \\
\hline Olax acuminata Wall. ex Benth. & Capsul gach & $\mathrm{Sj}, \mathrm{Wl}$ & Shrub; w & Native MAR 108 & M \\
\hline O. nana Wall. ex Benth.\# & Unknown & $\mathrm{Sj}, \mathrm{Wl}$ & Shrub; w & Native $M A R 2598$ & M \\
\hline \multicolumn{6}{|l|}{ Santalaceae R. Br. } \\
\hline Santalum album $\mathrm{L}$. & Shet chandan & $\mathrm{Gr}$ & Tree, s; pl & Exotic MAR 2719 & Co \\
\hline \multicolumn{6}{|l|}{ Loranthaceae Juss. } \\
\hline Dendrophthoe falcata (L. f.) Etting. & Bajrangi & Op & Shrub, ps; w & Native $S S 470$ & M \\
\hline $\begin{array}{l}\text { Macrosolen cochinchinensis (Lour.) van } \\
\text { Tiegh. }\end{array}$ & Renda & Op & Shrub, ps; w & Native SS 471 & M \\
\hline Scurrula parasitica $\mathrm{L}$. & Porgacha & Op & Shrub,ps; w & Native $G M H 5072$ & M \\
\hline Viscum monoicum Roxb. ex DC. & Bhanda & Op & Herb, ps; w & Native GMH 5069 & M, Po \\
\hline \multicolumn{6}{|l|}{ Euphorbiaceae Juss. } \\
\hline Acalypha ciliata Forssk. & Unknown & $\mathrm{Fl}, \mathrm{Sj}$ & Herb, er; w & Native MAR 2599 & M \\
\hline A. indica $\mathrm{L}$. & Muktajhuri & $\mathrm{Bw}^{1}, \mathrm{Fl}, \mathrm{Gl}, \mathrm{Rs}$ & Herb, er; w & Native $S S 472$ & M, Po \\
\hline $\begin{array}{l}\text { Chrozophora plicata (Vahl) A Juss. ex } \\
\text { Spreng. }\end{array}$ & Khudiokra & $\mathrm{Fl}, \mathrm{Gl}$ & Herb, er; w & Exotic $S S 473$ & M \\
\hline $\begin{array}{l}\text { Codiaeum variegatum (L.) Rumph. ex A. } \\
\text { Juss. }\end{array}$ & Patabahar & $\mathrm{Gr}$ & Shrub; pl & Exotic MAR 2603 & $\mathrm{O}$ \\
\hline Croton bonplandianus Baill. & Bandhone & $\mathrm{Fl}, \mathrm{Gl}, \mathrm{Rs}$ & Herb, er; w & Exotic SS 240 & M \\
\hline C. caudatus Geiseler & Nanvantui & $\mathrm{Fl}, \mathrm{Sj}, \mathrm{Rs}$ & Shrub, sc; w & Native MAR 116 & $\mathrm{Fu}, \mathrm{M}$ \\
\hline Euphorbia antiquorum $\mathrm{L}$. & Bajvaran & $\mathrm{Gr}, \mathrm{Hs}, \mathrm{Ml}$ & Shrub; pl-w & Native $M A R 2604$ & M \\
\hline E. heterophylla $\mathrm{L}$. & Baradudhia & $\mathrm{Fl}, \mathrm{Gl}, \mathrm{Rs}$ & Herb, pr; w & Exotic MAR 2605 & M \\
\hline E. hirta $\mathrm{L}$. & Dudhia & $\mathrm{Bw}^{1}, \mathrm{Fl}, \mathrm{Gl}, \mathrm{Rs}$ & Herb, pr; w & Exotic SS 127 & M \\
\hline E. milii Des Moul. & Kata mukut & $\mathrm{Gr}$ & Shrub; pl & Exotic MAR 2720 & $\mathrm{O}$ \\
\hline E. neriifolia $\mathrm{L}$. & Manosha sij & $\mathrm{Gr}, \mathrm{Hs}, \mathrm{Ml}$ & Shrub; pl-w & Exotic GMH 5097 & $\mathrm{O}, \mathrm{M}$ \\
\hline E. pulcherrima Willd. ex Klotzsch & Lalpata & Gr, Hs, Rs & Shrub; pl & Exotic $G M H 5065$ & $\mathrm{O}$ \\
\hline E. tithymaloides L. & Rangchita & $\mathrm{Gr}, \mathrm{Hs}$ & Herb, er; pl-w & Exotic GMH 5082 & M \\
\hline E. thymifolia $\mathrm{L}$. & Swetkerui & $\mathrm{Fl}, \mathrm{Gl}, \mathrm{Rs}$ & Herb, pr; w & Exotic $M A R 80$ & M \\
\hline Excoecaria cochinchinensis Lour. & Lailimajnu & Gr, Rs & Shrub; pl & Exotic $G M H 5079$ & $\mathrm{O}$ \\
\hline $\begin{array}{l}\text { Hevea brasiliensis (Willd. ex A.Juss.) } \\
\text { Müll.Arg. }\end{array}$ & Rubber & $\mathrm{Gr}$ & Tree, $1 ;$ pl & Exotic MAR 2606 & $\mathrm{M}, \mathrm{R}$ \\
\hline Jatropha gossypiifolia $\mathrm{L}$. & Lalbherenda & $\mathrm{Fl}, \mathrm{Rs}$ & Shrub; w & Exotic $S S 241$ & M \\
\hline J. integerrima Jacq. & Jayoti & $\mathrm{Fl}, \mathrm{Rs}$ & Shrub; w & Exotic MAR 2607 & $\mathrm{O}$ \\
\hline J. podagrica Hook. & Bagbherenda & Gr, Rs & Shrub; pl & Exotic MAR 2608 & $\mathrm{O}$ \\
\hline Mallotus nudiflorus (L.) Kulju \& Welzen & Medda & $\mathrm{Wl}$ & Tree, 1 ; w & Native $S S 475$ & $\mathrm{M}, \mathrm{T}$ \\
\hline M. philippensis (Lam.) Müll.-Arg. & Sinduri & $\mathrm{Sj}, \mathrm{Wl}$ & Shrub; w & Native SS 476 & Dy, M \\
\hline M. repandus (Willd.) Müll. Arg. & Gunti & $\mathrm{Sj}, \mathrm{Wl}$ & Shrub, sc; w & Native MAR 2609 & M \\
\hline Manihot esculenta Crantz + & Kasava & $\mathrm{Gr}, \mathrm{Hs}$ & Tree, s; pl & Exotic SSS 2058 & Ed, M \\
\hline Ricinus communis $\mathrm{L}$. & Bherenda & $\mathrm{Fl}, \mathrm{Hs}$ & Shrub; w & Exotic SS 477 & $\mathrm{M}, \mathrm{Oy}$ \\
\hline
\end{tabular}


Table 1 contd.

\begin{tabular}{|c|c|c|c|c|c|}
\hline Scientific name & Bangla name & Habitat & Habit & Origin RSE & Use \\
\hline Suregada multiflora (A. Juss.) Baill. ++ & Ban naringa & $\mathrm{Wl}$ & Tree, s; w & Native MAR 101 & Fw, M \\
\hline Tragia hispida Willd. & Bichuti & $\mathrm{Sj}$ & Herb, er; w & Native MAR 102 & M \\
\hline \multicolumn{6}{|l|}{ Phyllanthaceae Martinov } \\
\hline Antidesma acidum Retz. & Multa & $\mathrm{Sj}, \mathrm{Wl}$ & Shrub; w & Native MAR 160 & Ed, M \\
\hline A. bunius (L.) Spreng. & Banshialbuka & Gr & Tree, s; pl & Native $M A R 2600$ & Ed, M \\
\hline A. ghaesembilla Gaertn. & Khudijam & $\mathrm{Sj}, \mathrm{Wl}$ & Tree, s; w & Native $M A R 2601$ & Fr, M \\
\hline A. montanum Blume & Shialbuka & $\mathrm{Sj}, \mathrm{Wl}$ & Tree, s; w & Native SS 486 & Ed, M \\
\hline $\begin{array}{l}\text { Aporosa octandra (Buch.-Ham. ex D. } \\
\text { Don) Vickery }\end{array}$ & Patkhorolla & $\mathrm{Sj}, \mathrm{Wl}$ & Tree, s; w & Native SS 293 & Dy, Fw \\
\hline Baccaurea ramiflora Lour. & Latkan & $\mathrm{Gr}, \mathrm{Hs}$ & Tree, m; pl-w & Native SSS 2061 & Fr, M \\
\hline Breynia vitis-idaea (Burm. f.) Fisch. & Vitasalpoti & $\mathrm{Bw}^{1}, \mathrm{Sj}, \mathrm{Wl}$ & Tree, s; w & Native SS 239 & M \\
\hline Bridelia stipularis (L.) Blume & Kalasikori & $\mathrm{Sj}, \mathrm{Wl}$ & Shrub; w & Native SS 186 & Ed, M \\
\hline B. tomentosa Blume & Khoi & $\mathrm{Sj}, \mathrm{Wl}$ & Tree, s; w & Native $M A R 2602$ & Ed, M \\
\hline Flueggea virosa (Roxb. ex Willd.) Royle & Khaukra & $\mathrm{Sj}, \mathrm{Wl}$ & Shrub; w & Native SS 379 & M \\
\hline Phyllanthus acidus (L.) Skeels & Arboroi & $\mathrm{Gr}, \mathrm{Hs}$ & Tree, s; pl & Exotic SSS 2059 & Fr, M \\
\hline P. amarus Schumach. \&Thonn. & Bhuiamla & $\mathrm{Bw}^{1}, \mathrm{Fl}, \mathrm{Gl}$ & Herb, er; w & Exotic SS 178 & M \\
\hline P. emblica L. & Amloki & $\mathrm{Gr}, \mathrm{Hs}, \mathrm{Rs}$ & Tree, s; pl & Native SSS 2060 & Fr, M \\
\hline $\begin{array}{l}\text { P. multilocularis (Roxb. ex Willd.) Müll. } \\
\text { Arg. }\end{array}$ & Paniatori & $\mathrm{Fl}, \mathrm{Sj}$ & Shrub; w & Native $S S 474$ & M \\
\hline P. niruri L. & Bhuiamla & $\mathrm{Bw}^{1}, \mathrm{Fl}, \mathrm{Gl}$ & Herb, er; w & Exotic MAR 95 & M \\
\hline P. reticulatus Poir. & Chitki & $\mathrm{Bw}^{1}, \mathrm{Fl}, \mathrm{Sj}$ & Shrub; w & Native SS 197 & $\mathrm{Fu}, \mathrm{M}$ \\
\hline P. urinaria $\mathrm{L}$. & Kalochitki & $\mathrm{Fl}, \mathrm{Gl}$ & Herb, er; w & Native $M A R 96$ & M \\
\hline P. virgatus $\mathrm{G}$. Forst. & Sarnapati & $\mathrm{Bw}^{1}, \mathrm{Fl}, \mathrm{Gl}$ & Herb, er; w & Native SS 244 & M \\
\hline \multicolumn{6}{|l|}{ Putranjivaceae Endl. } \\
\hline \multicolumn{6}{|l|}{ Rhamnaceae Juss. } \\
\hline Gouania leptostachya DC. & Harjengagota & $\mathrm{Sj}, \mathrm{Wl}$ & Shrub, sc; w & Native $M A R 81$ & M \\
\hline Sarcomphalus mauritianus (Lam.) Raf. & Boroi & Gr, Hs, Wl & Tree, m; pl-w & Native SS 220 & Fr, Fw \\
\hline Zizyphus oenoplia (L.) Mill. & Bonboroi & $\mathrm{Sj}, \mathrm{Wl}$ & Shrub, sc; w & Native $S S 225$ & $\mathrm{Fu}, \mathrm{M}$ \\
\hline Z. rugosa Lam. & Jangliboroi & $\mathrm{Sj}, \mathrm{Wl}$ & Shrub; w & Native MAR 102 & M \\
\hline \multicolumn{6}{|l|}{ Leeaceae Dumort. } \\
\hline Leea asiatica $($ L.) Ridsdale & Banchalita & $\mathrm{Sj}, \mathrm{Wl}$ & Shrub; w & Native SS 389 & M \\
\hline \multicolumn{6}{|l|}{ Vitaceae Juss. } \\
\hline Ampelocissus latifolia (Roxb.) Planch. & Angur lata & $\mathrm{Bw}^{2}, \mathrm{Wl}$ & Herb, vi; w & Native $S S 478$ & M \\
\hline Causonis trifolia (L.) Mabb. \& J. Wen & Amal lata & $\mathrm{Bw}^{1}, \mathrm{Sj}, \mathrm{Wl}$ & Herb, vi; w & Native SS 479 & $\mathrm{Fd}, \mathrm{M}$ \\
\hline Cissus adnata Roxb. & Bhatia lata & $\mathrm{Sj}, \mathrm{Wl}$ & Herb, vi; w & Native $M A R 274$ & M \\
\hline C. quadrangularis $\mathrm{L}$. & Harjora & $\mathrm{Gr}, \mathrm{Fl}, \mathrm{Hs}$ & Herb, vi; w & Native $M A R 2610$ & M \\
\hline C. repens Lam. & Marmaria pata & $\mathrm{Sj}, \mathrm{Wl}$ & Herb, vi; w & Native $S S 480$ & M \\
\hline $\begin{array}{l}\text { Tetrastigma angustifolium (Roxb.) } \\
\text { Planch. }\end{array}$ & Nekungriubi & $\mathrm{Sj}, \mathrm{Wl}$ & Herb, vi; w & Native SS 294 & M \\
\hline \multicolumn{6}{|l|}{ Malpighiaceae Juss. } \\
\hline Hiptage benghalensis (L.) Kurz & Madhabi lata & $\mathrm{Gr}, \mathrm{Hs}$ & Liana; pl & Native SSS 2001 & $\mathrm{O}$ \\
\hline Malpighia coccigera $\mathrm{L}$. & Kantamalpia & Gr & Shrub; pl & Exotic SSS 2002 & $\mathrm{O}$ \\
\hline \multicolumn{6}{|l|}{ Polygalaceae Hoffmanns. \& Link } \\
\hline Polygala erioptera DC. & Teradudhi & $\mathrm{Fl}, \mathrm{Gl}$ & Herb, pr; w & Native MAR 110 & M \\
\hline
\end{tabular}


Table 1 contd.

\begin{tabular}{|c|c|c|c|c|c|}
\hline \multicolumn{6}{|l|}{ Sapindaceae Juss. } \\
\hline Cardiospermum halicacabum $\mathrm{L}$. & Lataphutki & $\mathrm{Fl}, \mathrm{Sj}$ & Herb, vi; w & Native $S S 481$ & M \\
\hline Dimocarpus longan Lour. ++ & Kathlitchu & $\mathrm{Gr}, \mathrm{Hs}$ & Tree, $\mathrm{m} ; \mathrm{pl}$ & Native $M A R 2611$ & Fr \\
\hline Lepisanthes rubiginosa (Roxb.) Leenh. & Horina & $\mathrm{Fl}, \mathrm{Gr}, \mathrm{Wl}$ & Tree, s; w & Native $G M H 5075$ & Fr, Fw \\
\hline Nephelium lappaceum L. + & Rambutan & $\mathrm{Gr}$ & Tree, $\mathrm{m} ; \mathrm{pl}$ & Exotic $M A R 2612$ & Fr \\
\hline Litchi chinensis Sonn. & Litchu & $\mathrm{Gr}, \mathrm{Hs}$ & Tree, $\mathrm{m} ; \mathrm{pl}$ & Exotic SSS 2062 & $\mathrm{Fr}$ \\
\hline Sapindus saponaria $\mathrm{L} .+$ & Ritha & $\mathrm{Gr}$ & Tree, s; pl & Exotic GMH 5043 & $\mathrm{M}, \mathrm{Co}$ \\
\hline Schleichera oleosa (Lour.) Merr. ++ & Kusum gach & $\mathrm{Gr}$ & Tree, $\mathrm{s} ; \mathrm{pl}$ & Native $M A R 2613$ & Dy, M \\
\hline \multicolumn{6}{|l|}{ Burseraceae Kunth } \\
\hline Canarium resiniferum Bruce ex King ++ & Dhup & Gr, Wl & Tree, $1 ; \mathrm{pl}$ & Native $M A R 2721$ & M \\
\hline \multicolumn{6}{|l|}{ Anacardiaceae R. Br. } \\
\hline Anacardium occidentale L. & Kaju badam & $\mathrm{Gr}, \mathrm{Hs}, \mathrm{Rs}$ & Tree, $1 ; \mathrm{pl}$ & Exotic $M A R 2614$ & Fr \\
\hline Lannea coromandelica (Houtt.) Merr. & Jiga & $\mathrm{Bw}^{2}, \mathrm{Fl}, \mathrm{Ml}, \mathrm{Wl}$ & Tree, s; w & Native GMH 5053 & Fn, Gu \\
\hline Mangifera indica $\mathrm{L}$. & Aam & $\mathrm{Gr}, \mathrm{Hs}, \mathrm{Wl}$ & Tree, $1 ;$ pl-w & Exotic $S S 482$ & Fr, $\mathrm{T}$ \\
\hline Spondias dulcis Parkinson & Amrah & $\mathrm{Gr}, \mathrm{Hs}$ & Tree, $1 ; \mathrm{pl}$ & Exotic SSS 2063 & Fr \\
\hline \multicolumn{6}{|l|}{ Meliaceae Juss. } \\
\hline Azadirachta indica A. Juss. & Neem & Hs, Rs, Wl & Tree, $\mathrm{m} ; \mathrm{pl}-\mathrm{w}$ & Native SS 391 & M \\
\hline $\begin{array}{l}\text { Aphanamixis polystachya (Wall.) R. } \\
\text { Parker }\end{array}$ & Pithraj & Hs, W1 & Tree, m; w & Native SSS 2064 & $\mathrm{M}, \mathrm{Oy}$ \\
\hline Chukrasia tabularis A.Juss. & Chikrassi & Gr, W1 & Tree, $1 ; \mathrm{pl}$ & Native SSS 2065 & Dy, T \\
\hline Dysoxylum excelsum Blume & Ramta rata & $\mathrm{Gr}$, & Tree, $1 ; \mathrm{pl}$ & Native $M A R 2615$ & $\mathrm{~T}, \mathrm{M}$ \\
\hline Khaya anthotheca (Welw.) C. DC. + & Lombu & Gr, Rs & Tree, $1 ; \mathrm{pl}$ & Exotic $M A R 2616$ & $\mathrm{~T}$ \\
\hline Melia azedarach $\mathrm{L}$. & Goranim & Rs, Wl & Tree, $\mathrm{m} ; \mathrm{pl}$ & Native GMH 5085 & $\mathrm{~T}, \mathrm{M}$ \\
\hline Swietenia macrophylla King & Bara Mehagani & Gr, Hs, Rs & Tree, $1 ;$ pl-w & Exotic $M A R 2617$ & $\mathrm{~T}$ \\
\hline S. mahagoni (L.) Jacq. & Mehagani & $\mathrm{Gr}, \mathrm{Hs}, \mathrm{Rs}$ & Tree, $1 ;$ pl-w & Exotic $M A R 143$ & $\mathrm{~T}$ \\
\hline \multicolumn{6}{|l|}{ Rutaceae Juss. } \\
\hline Aegle marmelos (L.) Corrêa & Bel & Hs, W1 & Tree, m; pl-w & Native $S S 403$ & Fr, M \\
\hline Feronia limonia (L.) Swingle & Kadbel & Gr, Hs & Tree, $\mathrm{m} ; \mathrm{pl}$ & Native SSS 2066 & Fr \\
\hline Citrus aurantiifolia (Christm.) Swingle & Lebu & $\mathrm{Gr}, \mathrm{Hs}$ & Shrub; pl & Exotic SSS 2067 & $\mathrm{Fr}$ \\
\hline C. hystrix DC. ++ & Satkora & $\mathrm{Gr}$ & Tree, s; pl & Native $M A R 2618$ & Fr, M \\
\hline C. limon (L.) Osbeck & Gora lebu & $\mathrm{Gr}$ & Tree, $\mathrm{s} ; \mathrm{pl}$ & Exotic $M A R 2619$ & Fr, Pi \\
\hline C. maxima (Burm.) Osbeck & Batabilebu & $\mathrm{Gr}, \mathrm{Hs}$ & Tree, s; pl & Exotic GMH 5011 & $\mathrm{Fr}$ \\
\hline $\begin{array}{l}\text { Clausena heptaphylla (Roxb.) Wight \& } \\
\text { Arn. }\end{array}$ & Pan mouri & $\mathrm{Gr}$ & Shrub; pl & Native $G M H 5095$ & M \\
\hline Glycosmis pentaphylla (Retz.) A. DC. & Datmajoni & $\mathrm{Fl}, \mathrm{Sj}, \mathrm{Wl}$ & Shrub; w & Native SS 276 & $\mathrm{Fu}, \mathrm{M}$ \\
\hline Murraya koenigii (L.) Spreng. & Curry patta & $\mathrm{Fl}, \mathrm{Gr}, \mathrm{Wl}$ & Tree, s; pl-w & Native $M A R 91$ & $\mathrm{M}, \mathrm{Sp}$ \\
\hline M. paniculata (L.) Jack & Kamini & Gr, Rs, W1 & Tree, s;pl-w & Native $G M H 5021$ & $\mathrm{M}, \mathrm{O}$ \\
\hline Zanthoxylum rhetsa (Roxb.) DC. & Bajna & $\mathrm{Fl}, \mathrm{Wl}$ & Tree, m; w & Native $S S 278$ & $\mathrm{M}, \mathrm{Oy}$ \\
\hline \multicolumn{6}{|l|}{ Oxalidaceae R. Br. } \\
\hline Averrhoa bilimbi $\mathrm{L}$. & Bilimbi & Gr, Hs & Tree, $\mathrm{s} ; \mathrm{pl}$ & Exotic $G M H 5063$ & $\mathrm{Fr}$ \\
\hline A. carambola $\mathrm{L}$. & Kamranga & $\mathrm{Gr}, \mathrm{Hs}$ & Tree, s; pl & Exotic GMH 5041 & Fr \\
\hline Oxalis corniculata $\mathrm{L}$. & Amrul & $\mathrm{Bw}^{1}, \mathrm{Gl}, \mathrm{Rs}$ & Herb, pr; w & Exotic $M A R 92$ & $\mathrm{M}, \mathrm{Vg}$ \\
\hline O. debilis Kunth & Golapi amrul & $\mathrm{Gr}, \mathrm{Hs}, \mathrm{Fl}$ & Herb, pr; pl-w & Exotic $M A R 2621$ & $\mathrm{O}$ \\
\hline \multicolumn{6}{|l|}{ Balsaminaceae A. Rich. } \\
\hline Impatiens balsamina L. & Dopati & $\mathrm{Gr}, \mathrm{Rs}$ & Herb, er; pl & Exotic SSS 2198 & $\mathrm{O}$ \\
\hline
\end{tabular}


Table 1 contd.

\begin{tabular}{|c|c|c|c|c|c|}
\hline Scientific name & Bangla name & Habitat & Habit & Origin RSE & Use \\
\hline \multicolumn{6}{|l|}{ Araliaceae Juss. } \\
\hline Polyscias fruticosa (L.) Harms & Tikosaya pata & Gr & Shrub; pl & Exotic SSS 2199 & $\mathrm{M}, \mathrm{O}$ \\
\hline P. scutellaria (Burm. f.) Fosberg, & Balbusaya pata & $\mathrm{Gr}$ & Shrub; pl & Exotic MAR 2622 & $\mathrm{M}, \mathrm{O}$ \\
\hline \multicolumn{6}{|l|}{ Apiaceae Lindl. } \\
\hline Centella asiatica (L.) Urb. & Thankuni & $\mathrm{Fl}, \mathrm{Gl}$ & Herb, cr; w & Native $S S 360$ & $\mathrm{M}, \mathrm{Vg}$ \\
\hline Coriandrum sativum $\mathrm{L}$. & Dhonia & $\mathrm{Fl}, \mathrm{Gr}, \mathrm{Hs}$ & Herb, er; cv & Exotic MAR 2623 & $\mathrm{M}, \mathrm{Sp}$ \\
\hline Eryngium foetidum $\mathrm{L}$. & Bilatedhoneya & $\mathrm{Gr}, \mathrm{Hs}$ & Herb, er; w & Exotic $G M H 5162$ & $\mathrm{M}, \mathrm{Sp}$ \\
\hline Foeniculum vulgare Mill. & Mouri & Hs & Herb, er; cv & Exotic MAR 2624 & $\mathrm{M}, \mathrm{Sp}$ \\
\hline Oenanthe benghalensis Benth. \& Hook. f. & Bon-dhonia & $\mathrm{Fl}, \mathrm{Gl}, \mathrm{Ml}$ & Herb, er; w & Native $S S S 2068$ & M \\
\hline \multicolumn{6}{|l|}{ Gentianaceae Juss. } \\
\hline $\begin{array}{l}\text { Canscora alata (Roth ex Roem. \& } \\
\text { Schult.) Wall. }\end{array}$ & Dhankuni & G1 & Herb, er; w & Native SS 170 & M \\
\hline \multicolumn{6}{|l|}{ Apocynaceae Juss. } \\
\hline Aganosma heynei (Spreng.) ined. & Malati lata & $\mathrm{Gr}$ & Liana; $\mathrm{pl}$ & Exotic MAR 2722 & $\mathrm{M}, \mathrm{O}$ \\
\hline Allamanda cathartica $\mathrm{L}$. & Ghonta phul & $\mathrm{Gr}, \mathrm{Hs}, \mathrm{Rs}$ & Shrub; pl & Exotic SSS 2068 & $\mathrm{O}$ \\
\hline Alstonia scholaris (L.) R. Br. & Chhatim & Rs, Wl & Tree, 1; pl-w & Native $S S 236$ & M \\
\hline Calotropis gigantea (L.) W.T. Aiton & Akondo & Ml, Rs & Shrub; w & Native SSS 2185 & $\mathrm{Fb}, \mathrm{M}$ \\
\hline Carissa carandas $\mathrm{L}$. & Karamcha & $\mathrm{Gr}, \mathrm{Hs}, \mathrm{Sj}$ & Shrub; pl-w & Native SSS 2069 & Fr, $\mathrm{Pc}$ \\
\hline Cascabela thevetia (L.) Lippold & Kolkey phul & $\mathrm{Gr}, \mathrm{Hs}$ & Tree, s; pl & Exotic MAR 2626 & $\mathrm{M}, \mathrm{O}$ \\
\hline Catharanthus roseus (L.) G. Don & Noyantara & $\mathrm{Bw}^{1}, \mathrm{Gr}, \mathrm{Hs}, \mathrm{Rs}$ & Herb, er; pl-w & Exotic $G M H 5051$ & $\mathrm{M}, \mathrm{O}$ \\
\hline Cryptostegia grandiflora Roxb. ex R. Br. & Kriptoran & Gr & Shrub; pl & Exotic MAR 2627 & M \\
\hline Hemidesmus indicus (L.) R. Br. ex Schult. & Anantomul & $\mathrm{Fl}, \mathrm{Gl}$ & Shrub; w & Native $M A R 2628$ & $\mathrm{Fb}, \mathrm{M}$ \\
\hline Holarrhena pubescens Wall. ex G. Don & Kurchi & $\mathrm{Gr}, \mathrm{Sj}, \mathrm{Wl}$ & Tree, s; w & Native SS 103 & Fw, M \\
\hline Ichnocarpus frutescens (L.) Aiton & Parallia lata & $\mathrm{Bw}^{1}, \mathrm{Fl}, \mathrm{Sj}, \mathrm{Wl}$ & Liana; w & Native SS 102 & $\mathrm{Fb}, \mathrm{M}$ \\
\hline Nerium oleander L. & Rakta karobi & $\mathrm{Gr}, \mathrm{Hs}, \mathrm{Rs}$ & Tree, s; pl & Exotic SSS 2070 & $\mathrm{O}$ \\
\hline Plumeria alba $\mathrm{L}$. & Shada kathgolap & $\mathrm{Gr}, \mathrm{Hs}, \mathrm{Rs}$ & Tree, $\mathrm{m} ; \mathrm{pl}$ & Exotic MAR 2629 & $\mathrm{O}$ \\
\hline P. obtusa $\mathrm{L}$. & Gorur-champa & Gr & Tree, $\mathrm{m} ; \mathrm{pl}$ & Exotic MAR 2630 & $\mathrm{O}$ \\
\hline P. pudica Jacq. & Nag dahur & $\mathrm{Gr}$ & Tree, s; pl & Exotic MAR 2631 & $\mathrm{O}$ \\
\hline P. rubra $\mathrm{L}$. & Lal kathgolap & $\mathrm{Gr}, \mathrm{Hs}, \mathrm{Rs}$ & Tree, $\mathrm{m} ; \mathrm{pl}$ & Native MAR 2632 & $\mathrm{O}$ \\
\hline $\begin{array}{l}\text { Rauvolfia serpentina (L.) Benth. ex } \\
\text { Kurz\# }\end{array}$ & Sarpogondha & Gr, Wl & Shrub; pl-w & Native $M A R 99$ & M \\
\hline R. tetraphylla $\mathrm{L}$. & Sharpamul & $\mathrm{Gr}$ & Shrub; pl & Exotic $G M H 5073$ & M \\
\hline $\begin{array}{l}\text { Tabernaemontana divaricata (L.) R. Br. } \\
\text { ex Roem \& Schult. }\end{array}$ & Tagar & Gr, Rs, Sj, Wl & Shrub; w & Native SS 134 & $\mathrm{M}, \mathrm{O}$ \\
\hline \multicolumn{6}{|l|}{ Solanaceae Juss. } \\
\hline Capsicum аппиит $\mathrm{L}$. & Morich & $\mathrm{Gr}, \mathrm{Hs}$ & Herb, er; cv & Exotic SSS 2074 & $\mathrm{Sp}$ \\
\hline Cestrum diurnum $\mathrm{L}$. & Hasnahena & Hs & Shrub; pl & Exotic $G M H 5163$ & $\mathrm{O}$ \\
\hline Datura stramonium L. & Sada dhutra & $\mathrm{Fl}, \mathrm{Gr}, \mathrm{Rs}$ & Shrub; w & Exotic SSS 2071 & M \\
\hline Nicotiana plumbaginifolia Viv. & Ban tamak & $\mathrm{Bw}^{1}, \mathrm{Fl}, \mathrm{Gl}, \mathrm{Rs}$ & Herb, er; w & Exotic SS 283 & M \\
\hline Petunia hybrida E. Vilm. & Petunia & Gr, Rs & Herb, er; pl & Exotic SSS 2200 & $\mathrm{O}$ \\
\hline Physalis angulata $\mathrm{L}$. & Futka & $\mathrm{Fl}, \mathrm{Gl}, \mathrm{Rs}$ & Herb, er; w & Exotic SS 284 & M \\
\hline Solanum americanum Mill. & Tit-begun & $\mathrm{Bw}^{1}, \mathrm{Fl}, \mathrm{Gl}, \mathrm{Rs}$ & Herb, er; w & Exotic SSS 2072 & M \\
\hline S. melongena $\mathrm{L}$. & Begun & $\mathrm{Gr}, \mathrm{Hs}$ & Shrub; w & Exotic SSS 2073 & $\mathrm{Vg}$ \\
\hline S. torvum $\mathrm{Sw}$. & Gota begun & $\mathrm{Fl}, \mathrm{Sj}$, Rs & Shrub; w & Exotic SS 195 & $\mathrm{M}, \mathrm{Vg}$ \\
\hline S. violaceum Ortega & Phutki begun & $\mathrm{Bw}^{1}, \mathrm{Fl}, \mathrm{Sj}, \mathrm{Rs}$ & Shrub; w & Native SS 404 & M \\
\hline
\end{tabular}


Table 1 contd.

\begin{tabular}{|c|c|c|c|c|c|}
\hline Scientific name & Bangla name & Habitat & Habit & Origin RSE & Use \\
\hline S. virginianum $\mathrm{L}$. & Kantakari & Fl, Rs & Herb, pr; w & Native MAR 111 & $\mathrm{M}$ \\
\hline \multicolumn{6}{|l|}{ Convolvulaceae Juss. } \\
\hline Aniseia martinicensis (Jacq.) Choisy & Shadamati & $\mathrm{Fl}, \mathrm{Gl}$ & Herb, vi; w & Exotic MAR 129 & M \\
\hline Argyreia capitiformis (Poir.) Ooststr. & Bijtarak & $\mathrm{Sj}, \mathrm{Wl}$ & Shrub, sc; w & Native $M A R 130$ & M \\
\hline $\begin{array}{l}\text { Camonea umbellata (L.) A.R. Simões \& } \\
\text { Staples }\end{array}$ & Goria lata & $\mathrm{Fl}, \mathrm{Gl}, \mathrm{Rs}$ & Herb, vi; w & Exotic $M A R 90$ & M \\
\hline $\begin{array}{l}\text { C. vitifolia (Burm. f.) A.R. Simões \& } \\
\text { Staples }\end{array}$ & Korma lata & $\mathrm{Sj}, \mathrm{Wl}$ & Herb, vi; w & Native MAR 215 & M \\
\hline Evolvulus nummularius (L.) L. & Bhui okra & $\mathrm{Bw}^{1}, \mathrm{Fl}, \mathrm{Gl}, \mathrm{Rs}$ & Herb, cr; w & Exotic SS 483 & $\mathrm{M}, \mathrm{Sb}$ \\
\hline Ipomoea aquatica Forssk. & Kalmishak & $\mathrm{Fl}, \mathrm{Wtl}$ & Herb, cr; pl-w & Native $S S 377$ & $\mathrm{Vg}$ \\
\hline I. fistulosa Mart. ex Choisy & Dhol kolmi & $\mathrm{Fl}, \mathrm{Ml}$ & Shrub; w & Exotic MAR83 & $\mathrm{Pp}, \mathrm{Sb}$ \\
\hline I. obscura (L.) Ker Gawl. & Kura kalmi & Gr, Rs & Herb, vi; w & Native $M A R 2723$ & Fo, $\mathrm{M}$ \\
\hline I. quamoclit $\mathrm{L}$. & Kunja lata & $\mathrm{Gr}, \mathrm{Hs}$ & Herb, vi, pl & Exotic $M A R 2633$ & $\mathrm{O}$ \\
\hline I. tricolor Cav. & Morning glory & $\mathrm{Gr}, \mathrm{Hs}$ & Herb, vi; pl & Exotic MAR 2634 & $\mathrm{O}$ \\
\hline $\begin{array}{l}\text { Xenostegia tridentata (L.) D.F. Austin \& } \\
\text { Staples ++ }\end{array}$ & Prasarini & Gl, Rs & Herb, vi; w & Native $M A R 2724$ & M \\
\hline \multicolumn{6}{|l|}{ Cuscutaceae Dumort. } \\
\hline Cuscuta chinensis Lam. & $\begin{array}{l}\text { Chinese sharno } \\
\text { lata }\end{array}$ & Op & Herb, ps; w & Native SSS 2075 & M \\
\hline C. reflexa Roxb. & Sharno lata & Op & Herb, ps; w & Native SS 484 & M \\
\hline \multicolumn{6}{|l|}{ Menyanthaceae Dumort. } \\
\hline Nymphoides cristata (Roxb.) Kuntze & Chand mala & Wtl & Herb, fr; w & Native $M A R 2635$ & Ed \\
\hline N. hydrophylla (Lour.) Kuntze & Chand mona & Wtl & Herb, fr; w & Native MAR 161 & M \\
\hline N. indica (L.) Kuntze & Panchuli mala & Wtl & Herb, fr; w & Native SS 392 & Ed, M \\
\hline \multicolumn{6}{|l|}{ Polemoniaceae Juss. } \\
\hline Phlox drummondii Hook. + & Flox & Gr, Rs & Herb, er; pl & Exotic $M A R 2730$ & $\mathrm{O}$ \\
\hline \multicolumn{6}{|l|}{ Hydroleaceae R. Br. ex Edwards } \\
\hline Hydrolea zeylanica (L.) Vahl & Kasschera & Wtl & Herb, pr; w & Native SS 169 & M \\
\hline \multicolumn{6}{|l|}{ Boraginaceae Juss. } \\
\hline Heliotropium indicum $\mathrm{L}$. & Hatisur & $\mathrm{Fl}, \mathrm{Gl}, \mathrm{Rs}$ & Herb, er; w & Exotic SS 485 & M \\
\hline Cordia dichotoma $\mathrm{G}$. Forst. & Bohola & $\mathrm{Sj}, \mathrm{Wl}$ & Tree, $\mathrm{m} ; \mathrm{w}$ & Native GMH 5083 & $\mathrm{Gu}, \mathrm{M}$ \\
\hline \multicolumn{6}{|l|}{ Verbenaceae J. St.-Hil. } \\
\hline Duranta erecta $\mathrm{L}$. & Duranto & Gr, Ml, Rs & Shrub; pl-w & Exotic SSS 2075 & $\mathrm{O}$ \\
\hline Lantana camara $\mathrm{L}$. & Kutus kanta & $\mathrm{Bw}^{1}, \mathrm{Rs}, \mathrm{Sj}, \mathrm{Wl}$ & Shrub; w & Exotic SS 112 & M \\
\hline $\begin{array}{l}\text { Lippia alba (Mill.) N.E. Br. ex Britton \& } \\
\text { P. Wilson }\end{array}$ & Pichas-lakri & $\mathrm{Fl}, \mathrm{Sj}$ & Shrub; w & Exotic SSS 2076 & M \\
\hline Petrea volubilis L. + & Nilmoni lata & Gr & Shrub, sc; pl & Exotic $M A R 2636$ & $\mathrm{O}$ \\
\hline Phyla nodiflora (L.) Greene & Vuiokra & $\mathrm{Fl}, \mathrm{Gl}, \mathrm{Rs}$ & Herb, cr; w & Native SSS 2077 & M \\
\hline \multicolumn{6}{|l|}{ Lamiaceae Martinov } \\
\hline Anisomeles indica (L.) Kuntze. & Gobura & $\mathrm{Fl}, \mathrm{Wl}$ & Herb, er; w & Native $S S 135$ & M \\
\hline Clerodendrum indicum (L.) Kuntze & Bamunhatti & $\mathrm{Fl}, \mathrm{Sj}, \mathrm{Wl}$ & Shrub; w & Native $S S 216$ & M \\
\hline C. infortunatum $\mathrm{L}$. & Bhat & $\mathrm{Fl}, \mathrm{Sj}, \mathrm{Rs}, \mathrm{Wl}$ & Shrub; w & Native MAR 162 & M \\
\hline C. splendens G. Don & Shum bhat & $\mathrm{Gr}$ & Shrub; pl & Exotic MAR 2637 & M \\
\hline Gmelina arborea Roxb. & Gamari & Gr, Wl & Tree, $1 ;$ pl-w & Native SSS 2078 & $\mathrm{~T}$ \\
\hline Hyptis capitata Jacq. & Tata tokma & $\mathrm{Fl}, \mathrm{Rs}, \mathrm{Sj}$ & Herb, er; w & Exotic SS 137 & M \\
\hline H. suaveolens (L.) Poit. & Tokma & $\mathrm{Fl}, \mathrm{Rs}, \mathrm{Sj}$ & Herb, er; w & Exotic SSS 2079 & M \\
\hline
\end{tabular}


Table 1 contd.

\begin{tabular}{|c|c|c|c|c|c|}
\hline Scientific name & Bangla name & Habitat & Habit & Origin RSE & Use \\
\hline Leucas zeylanica (L.) W.T. Aiton & Dondokalosh & $\mathrm{Bw}^{1}, \mathrm{Fl}, \mathrm{Gl}, \mathrm{Rs}$ & Herb, er; w & Native $S S 256$ & M \\
\hline Leonurus sibiricus $\mathrm{L}$. & Rokto-dron & $\mathrm{Fl}, \mathrm{Rs}$ & Herb, er; w & Native $M A R 2638$ & M \\
\hline Ocimum basilicum $\mathrm{L}$. & Bantulsi & $\mathrm{Fl}, \mathrm{Gr}, \mathrm{Hs}$ & Herb, er; pl-w & Native SSS 2080 & M \\
\hline O. gratissimum $\mathrm{L}$. & Ram tulsi & $\mathrm{Gr}, \mathrm{Hs}$ & Herb, er; pl-w & Native SSS 2081 & M \\
\hline O. tenuiflorum $\mathrm{L}$. & Kalo tulsi & $\mathrm{Fl}, \mathrm{Gr}, \mathrm{Hs}$ & Herb, er; pl-w & Native SSS 2082 & M \\
\hline Pogostemon auricularius (L.) Hassk. & Aripachuli & $\mathrm{Fl}, \mathrm{Gl}, \mathrm{Rs}$ & Herb, er; w & Native $S S 258$ & M \\
\hline Plectranthus scutellarioides (L.) R. Br. & Coleus & $\mathrm{Bw}^{1}, \mathrm{Fl}, \mathrm{Gr}, \mathrm{Rs}$ & Herb, er; w & Exotic MAR 2640 & $\mathrm{O}$ \\
\hline Rotheca serrata (L.) Steane \& Mabb. & Bamanhati & $\mathrm{Sj}, \mathrm{Wl}$ & Shrub; w & Native $S S 217$ & M \\
\hline Salvia splendens Sellow ex Schult. & Lal sagi & Gr, Rs, Hs & Herb, er; pl & Exotic MAR 2641 & $\mathrm{O}$ \\
\hline Tectona grandis L. f. & Shegun & Rs, W1 & Tree, $1 ; \mathrm{pl}$ & Native SS 487 & $\mathrm{~T}$ \\
\hline Vitex negundo $\mathrm{L}$. & Nishinda & $\mathrm{Fl}, \mathrm{Sj}$, Rs & Shrub; w & Native GMH 5164 & M \\
\hline V. peduncularis Wall. ex Schauer & Goda & Gr, Wl & Tree, $\mathrm{m} ; \mathrm{pl}$ & Native MAR 2642 & $\mathrm{~T}$ \\
\hline Volkameria inermis $\mathrm{L}$. & Shita vat & Gr, Rs & Shrub; pl-w & Exotic GMH 5093 & $\mathrm{M}, \mathrm{O}$ \\
\hline \multicolumn{6}{|l|}{ Plantaginaceae Juss. } \\
\hline Antirrhinum majus $\mathrm{L}$. & Sonipati & Gr, Rs & Herb, er; pl & Exotic $M A R 2725$ & $\mathrm{O}$ \\
\hline Limnophila aromatica (Lam.) Merr. & Pani korpur & $\mathrm{Fl}, \mathrm{Wtl}$ & Herb, pr; w & Native $G M H 5187$ & M \\
\hline L. chinensis (Osbeck) Merr. & Anguli ghash & $\mathrm{Fl}, \mathrm{Wtl}$ & Herb, pr; w & Native $G M H 5188$ & M \\
\hline L. heterophylla (Roxb.) Benth. & Patakutra & Wtl & Herb, fr; w & Native $S S 488$ & $\mathrm{M}, \mathrm{O}$ \\
\hline L. sessiliflora (Vahl) Blume & Bamonkeshori & Wtl & Herb, em; w & Native SS 489 & $\mathrm{Wp}$ \\
\hline Mecardonia procumbens (Mill.) Small & Micardan & $\mathrm{Fl}, \mathrm{Gl}, \mathrm{Rs}$ & Herb, pr; w & Exotic $G M H 5051$ & M \\
\hline Scoparia dulcis L. & Bondhone & $\mathrm{Bw}^{1}, \mathrm{Fl}, \mathrm{Gl}, \mathrm{Rs}$ & Herb, er; w & Exotic $S S 281$ & M \\
\hline \multicolumn{6}{|l|}{ Oleaceae Hoffmanns. \& Link } \\
\hline Jasminum grandiflorum $\mathrm{L}$. & Chameli & Gr & Shrub; pl & Native SSS 2083 & $\mathrm{M}, \mathrm{O}$ \\
\hline J. sambac (L.) Sol. & Jui, beli & Gr, Rs, Hs & Shrub; pl & Exotic SSS 2084 & $\mathrm{O}$ \\
\hline J. scandens (Retz.) Vahl & Paharijui & $\mathrm{Sj}, \mathrm{Wl}$ & Shrub; w & Native SS 203 & M \\
\hline Nyctanthes arbor-tristis $\mathrm{L}$. & Sheuli & $\mathrm{Gr}, \mathrm{Hs}$ & Tree, s; pl & Native SSS 2085 & $\mathrm{M}, \mathrm{O}$ \\
\hline \multicolumn{6}{|l|}{$\begin{array}{l}\text { Linderniaceae Borsch, Kai Müll. \& Eb. } \\
\text { Fisch. }\end{array}$} \\
\hline Bonnaya antipoda (L.) Druce & Zai ghas & $\mathrm{Fl}, \mathrm{Gl}, \mathrm{Rs}$ & Herb, pr; w & Native SS 222 & M \\
\hline Lindernia ciliata (Colsm.) Pennell & Bhui papri & $\mathrm{Fl}, \mathrm{Gl}, \mathrm{Rs}$ & Herb, pr; w & Native SS 490 & M \\
\hline L. crustacea (L.) F. Muell. & Chapra ghas & $\mathrm{Fl}, \mathrm{Gl}, \mathrm{Rs}$ & Herb, pr; w & Native SS 491 & M \\
\hline L. parviflora (Roxb.) Haines & Parvi chapra & $\mathrm{Fl}, \mathrm{Gl}, \mathrm{Rs}$ & Herb, pr; w & Native $M A R 2642$ & M \\
\hline L. procumbens (Krock.) Borbás & Bakpuspa & $\mathrm{Fl}, \mathrm{Gl}, \mathrm{Rs}$ & Herb, pr; w & Native $M A R 144$ & M \\
\hline L. rotundifolia (L.) Alston & Tan chapra & $\mathrm{Bw}^{1}, \mathrm{Fl}, \mathrm{Gl}, \mathrm{Rs}$ & Herb, pr; w & Native $M A R 87$ & M \\
\hline Torenia diffusa D. Don & Ushatoren & $\mathrm{Fl}, \mathrm{Rs}$ & Herb, pr; w & Native $S S 492$ & $\mathrm{O}$ \\
\hline \multicolumn{6}{|l|}{ Mazaceae Reveal } \\
\hline \multicolumn{5}{|l|}{ Acanthaceae Juss. } & M \\
\hline $\begin{array}{l}\text { Andrographis paniculata (Burm. f.) } \\
\text { Nees \# }\end{array}$ & Kalomegh & Gr, Hs, Wl & Herb, er; pl-w & Native $M A R 2643$ & M \\
\hline Asystasia gangetica $($ L.) T. Anderson ++ & Gangatara & $\mathrm{Fl}, \mathrm{Sj}$ & Herb, pr; w & Native $S S 132$ & M \\
\hline $\begin{array}{l}\text { Dicliptera paniculata (Forssk.) I. } \\
\text { Darbysh. }\end{array}$ & Nashabhanga & Rs, Gl, Wl & Herb, er; w & Native SS 348 & M \\
\hline Ecbolium ligustrinum (Vahl) Vollesen & Shial leza & $\mathrm{Fl}, \mathrm{Wl}$ & Herb, er; w & Native SSS 2086 & M \\
\hline Hygrophila erecta (Burm. f.) Hochr & Filareck & Wtl & Herb, er; w & Native MAR 107 & M \\
\hline H. hirta (Vahl) T. Anderson & Buripana & $\mathrm{Fl}, \mathrm{Gl}, \mathrm{Rs}$ & Herb, pr; w & Native $M A R 75$ & M \\
\hline
\end{tabular}


Table 1 contd.

\begin{tabular}{|c|c|c|c|c|c|}
\hline Scientific name & Bangla name & Habitat & Habit & Origin RSE & Use \\
\hline H. polysperma (Roxb.) T. Anderson & Alai kalai & $\mathrm{Fl}, \mathrm{Wtl}$ & Herb, pr; w & Native SSS 2087 & M \\
\hline Justicia adhatoda $\mathrm{L}$. & Basak & $\mathrm{Fl}, \mathrm{Gr}, \mathrm{Hs}, \mathrm{Sj}$ & Shurb; w & Native SS 275 & M \\
\hline J. gendarussa Burm.f. & Jagotmadan & $\mathrm{Fl}, \mathrm{Ml}, \mathrm{Sj}$ & Herb, er; pl-w & Native $M A R 84$ & Fn, M \\
\hline Nelsonia canescens (Lam.) Spreng. & Paramul & $\mathrm{Fl}, \mathrm{Gl}, \mathrm{Wl}$ & Herb, pr; w & Native SS 209 & M \\
\hline Phaulopsis imbricata (Forssk.) Sweet & Bhuibashak & $\mathrm{Bw}^{1}, \mathrm{Sj}, \mathrm{Wl}$ & Herb, pr; w & Native $M A R 93$ & M \\
\hline Ruellia tuberosa $\mathrm{L}$. & Chotpotey & $\mathrm{Fl}, \mathrm{Wl}$ & Herb, er; w & Exotic SS 351 & $\mathrm{M}, \mathrm{O}$ \\
\hline Rungia pectinata (L.) Nees & Pindi & $\mathrm{Bw}^{1}, \mathrm{Fl}, \mathrm{Gl}, \mathrm{Rs}$ & Herb, pr; w & Native GMH 5061 & M \\
\hline Staurogyne zeylanica Kuntze & Cylongyne & $\mathrm{Fl}$ & Herb, pr; w & Native $M A R 2644$ & M \\
\hline $\begin{array}{l}\text { Thunbergia grandiflora (Roxb. ex Rottl.) } \\
\text { Roxb. }\end{array}$ & Neel lata & $\mathrm{Ml}, \mathrm{Sj}, \mathrm{Wl}$ & Herb, vi; w & Native $M A R 2712$ & M \\
\hline T. mysorensis (Wight) T. Anderson & Basar lata & $\mathrm{Gr}$ & Herb, vi; pl & Exotic MAR 2645 & $\mathrm{O}$ \\
\hline \multicolumn{6}{|l|}{ Pedaliaceae R. Br. } \\
\hline Sesamum indicum $\mathrm{L}$. & Til & $\mathrm{Fl}, \mathrm{Hs}$ & Herb, er; pl-w & Native SSS 2088 & Oy \\
\hline \multicolumn{6}{|l|}{ Bignoniaceae Juss. } \\
\hline Bignonia magnifica $\mathrm{W}$. Bull & Unknown & $\mathrm{Gr}, \mathrm{Hs}$ & Liana; $\mathrm{pl}$ & Exotic MAR 2646 & $\mathrm{O}$ \\
\hline Jacaranda mimosifolia D. Don & Nil gulmohor & Gr, Rs & Tree, l; w & Exotic $M A R 2647$ & $\mathrm{O}$ \\
\hline Mansoa alliacea (Lam.) A.H. Gentry & Rasun lata & $\mathrm{Gr}, \mathrm{Hs}$ & Liana; pl & Exotic $M A R 2648$ & $\mathrm{O}$ \\
\hline Oroxylum indicum (L.) Kurz & Kanidingi & $\mathrm{Sj}, \mathrm{Wl}$ & Tree, m; w & Native SS 493 & M \\
\hline Parmentiera aculeata (Kunth) Seem. ++ & Mombati phal & $\mathrm{Gr}$ & Tree, $1 ; \mathrm{pl}$ & Native $M A R 2649$ & Ed, M \\
\hline Pyrostegia venusta (Ker Gawl.) Miers & Sonali lata & $\mathrm{Gr}$ & Liana; pl & Exotic $M A R 2650$ & $\mathrm{M}, \mathrm{O}$ \\
\hline $\begin{array}{l}\text { Tabebuia rosea (Bertol.) Bertero ex A. } \\
\text { DC. }+\end{array}$ & Tobebia & Gr & Tree, m; pl & Exotic $M A R 2651$ & $\mathrm{O}$ \\
\hline Tecoma stans (L.) Juss. ex Kunth + & Tecoma & Gr, Rs & Tree, s; pl & Exotic SSS 2089 & $\mathrm{O}$ \\
\hline $\begin{array}{l}\text { Spathodea campanulata P. Beauv. + } \\
\text { Lentibulariaceae Rich. }\end{array}$ & Rudra polash & Gr, Rs & Tree, $1 ; \mathrm{pl}$ & Exotic SSS 2090 & $\mathrm{M}, \mathrm{O}$ \\
\hline Utricularia aurea Lour. & Patajhajhi & Wtl & Herb, sm; w & Native $G M H 5165$ & $\mathrm{M}, \mathrm{Wp}$ \\
\hline \multicolumn{6}{|l|}{ Campanulaceae Juss. } \\
\hline Wahlenbergia marginata (Thunb.) A. DC. & Kardi & $\mathrm{Lb}, \mathrm{Wtl}$ & Herb, er; w & Native $M A R 2726$ & M \\
\hline \multicolumn{6}{|l|}{ Rubiaceae Juss. } \\
\hline Catunaregam spinosa (Thunb.) Tirveng. & Man kanta & $\mathrm{Bw}^{2}, \mathrm{Sj}, \mathrm{Wl}$ & Shrub; w & Native SS 494 & $\mathrm{Fu}, \mathrm{M}$ \\
\hline Coffea arabica $\mathrm{L}$. & Koffee & $\mathrm{Gr}$ & Shrub; pl & Exotic $S A K 2103$ & $\mathrm{Bv}, \mathrm{M}$ \\
\hline C. benghalensis B. Heyne ex Schult. & Bangla koffe & $\mathrm{Sj}, \mathrm{Wl}$ & Shrub; w & Native GMH 5071 & M \\
\hline $\begin{array}{l}\text { Dentella repens (L.) J.R. Forst. \& G. } \\
\text { Forst. }\end{array}$ & Bhuipat & $\mathrm{Fl}, \mathrm{Gl}$ & Herb, pr; w & Native $S S 400$ & M \\
\hline Gardenia coronaria Buch.-Ham. & Koinor & $\mathrm{Gr}$ & Tree, m; pl & Native $S A K 2062$ & $\mathrm{O}, \mathrm{T}$ \\
\hline G. jasminoides J. Ellis & Gondhoraj & $\mathrm{Gr}, \mathrm{Hs}$ & Shrub; pl & Native $M A R 2652$ & $\mathrm{M}, \mathrm{O}$ \\
\hline G. latifolia Aiton & Papra & $\mathrm{Gr}, \mathrm{Hs}$ & Shrub; pl & Native $M A R 2653$ & $\mathrm{O}$ \\
\hline Haldina cordifolia (Roxb.) Ridsdale & Haldu & $\mathrm{Gr}$ & Tree, m; pl & Native SS 495 & $\mathrm{M}, \mathrm{T}$ \\
\hline Hymenodictyon orixense (Roxb.) Mabb. & Bhuikadam & $\mathrm{Gr}$ & Tree, $1 ; \mathrm{pl}$ & Native $M A R 2654$ & $\mathrm{M}, \mathrm{T}$ \\
\hline Ixora coccinea $\mathrm{L}$. & Rangon & Gr, Rs, Hs & Shrub; pl & Native $S A K 2027$ & $\mathrm{O}$ \\
\hline I. cuneifolia Roxb. & Jangli rangon & $\mathrm{Sj}, \mathrm{Wl}$ & Shrub; w & Native $S A K 2020$ & M \\
\hline I. pavetta Andr. & Banrangon & $\mathrm{Sj}, \mathrm{Wl}$ & Shrub; w & Native SS 224 & M \\
\hline I. undulata Roxb. & Palkajui & $\mathrm{Sj}, \mathrm{Wl}$ & Shrub; w & Native $S S 273$ & M \\
\hline Meyna spinosa Roxb. ex Link & Katai & $\mathrm{Sj}, \mathrm{Wl}$ & Shrub; w & Native $M A R 2654$ & M \\
\hline Mitragyna parvifolia (Roxb.) Korth. & Phulkadam & $\mathrm{Gr}$ & Tree; $1 ; \mathrm{pl}$ & Native $M A R 2727$ & M \\
\hline Morinda angustifolia Roxb. & Pandusi & $\mathrm{Sj}, \mathrm{Wl}$ & Shrub; w & Native SS 274 & M \\
\hline
\end{tabular}


Table 1 contd.

\begin{tabular}{|c|c|c|c|c|c|}
\hline Scientific name & Bangla name & Habitat & Habit & Origin RSE & Use \\
\hline $\begin{array}{l}\text { Mussaenda erythrophylla Schumach. \& } \\
\text { Thonn. }\end{array}$ & Lal mussenda & $\mathrm{Gr}, \mathrm{Hs}$ & Shrub; pl & Exotic SSS 2091 & $\mathrm{O}$ \\
\hline M. frondosa $\mathrm{L}$. & Kalasona & $\mathrm{Gr}, \mathrm{Hs}$ & Shrub; pl & Native SSS 2092 & $\mathrm{O}$ \\
\hline M. philippica A. Rich. & Mussenda & $\mathrm{Gr}, \mathrm{Hs}$ & Shrub; pl & Exotic SSS 2093 & $\mathrm{O}$ \\
\hline Neolamarckia cadamba (Roxb.) Bosser & Kadom & $\mathrm{Bw}^{2}, \mathrm{Rs}, \mathrm{Wl}$ & Tree, $1 ; \mathrm{w}$ & Native $S S 498$ & $\mathrm{O}, \mathrm{T}$ \\
\hline Oldenlandia corymbosa $\mathrm{L}$. & Khet papra & $\mathrm{Bw}^{1}, \mathrm{Fl}, \mathrm{Gl}$ & Herb, pr; w & Native SS 496 & M \\
\hline O. diffusa (Willd.) Roxb. & Fussa papra & $\mathrm{Fl}, \mathrm{Gl}$ & Herb, pr; w & Native SS 497 & M \\
\hline O. verticillata $\mathrm{L}$. & Notapapra & $\mathrm{Fl}, \mathrm{Gl}$ & Herb, pr; w & Native $M A R 82$ & M \\
\hline Paederia foetida $\mathrm{L}$. & Gandhyabhaduli & $\mathrm{Gr}, \mathrm{Hs}, \mathrm{Sj}$ & Herb, vi; w & Native SS 499 & M \\
\hline Richardia scabra $\mathrm{L}$. & Nakli ipecac & $\begin{array}{l}\mathrm{Bw}^{1}, \mathrm{Fl}, \mathrm{Gl}, \mathrm{Rs} \\
\mathrm{Wl}\end{array}$ & Herb, pr; w & Exotic $S A K 2030$ & M \\
\hline Spermacoce articularis L. f. & Bahos & $\mathrm{Fl}, \mathrm{Gl}, \mathrm{Rs}, \mathrm{Wl}$ & Herb, pr; w & Native $S S 272$ & M \\
\hline $\begin{array}{l}\text { S. exilis (L.O. Williams) C.D. Adams ex } \\
\text { W.C. Burger \& C.M. Taylor }\end{array}$ & Baghajangla & $\begin{array}{l}\mathrm{Bw}^{1}, \mathrm{Fl}, \mathrm{Gl}, \mathrm{Rs}, \\
\mathrm{Wl}\end{array}$ & Herb, pr; w & Exotic $G M H 5168$ & M \\
\hline $\begin{array}{l}\text { Tamilnadia uliginosa (Retz.) Tirveng. \& } \\
\text { Sastre ++ }\end{array}$ & Piralu & $\mathrm{Sj}$ & Tree, s; w & Native $M A R 98$ & $\mathrm{Fu}, \mathrm{M}$ \\
\hline Vangueria madagascariensis J.F. Gmel. & Mainakata & $\mathrm{Sj}, \mathrm{Wl}$ & Shrub; w & Exotic $S S 402$ & M \\
\hline \multicolumn{6}{|l|}{ Asteraceae Bercht. \& J. Presl } \\
\hline Acmella calva (DC.) R.K. Jansen. & Surjakonnya & $\mathrm{Bw}^{1}, \mathrm{Fl}, \mathrm{Gr}, \mathrm{Rs}$ & Herb, pr; w & Native $S S 144$ & M \\
\hline $\begin{array}{l}\text { A. paniculata (Wall. ex DC.) R.K. } \\
\text { Jansen }\end{array}$ & Mahatitinga & Gr, Rs & Herb, pr; w & Native $S A K 2041$ & M \\
\hline Ageratum conyzoides $(\mathrm{L}.) \mathrm{L}$. & Fulkuri & $\mathrm{Bw}^{1}, \mathrm{Fl}, \mathrm{Rs}, \mathrm{Sj}$ & Herb, er; w & Exotic SAK 2077 & M \\
\hline Bidens pilosa $\mathrm{L}$. & Bidenlosa & Gr, Wl & Herb, er; w & Exotic $S A K 2045$ & $\mathrm{O}$ \\
\hline Blumea densiflora DC. & Nagorfuli & $\mathrm{Fl}, \mathrm{Gl}, \mathrm{Rs}$ & Herb, er; w & Native $M A R 146$ & M \\
\hline B. lacera (Burm.f.) DC. & Barokukshim & $\mathrm{Fl}, \mathrm{Gl}, \mathrm{Rs}$ & Herb, er; w & Native $S A K 2026$ & M \\
\hline B. membranacea Wall. ex DC. & Kukurshinga & $\mathrm{Fl}, \mathrm{Gl}, \mathrm{Rs}$ & Herb, er; w & Native $S S 365$ & M \\
\hline B. oxyodonta DC. & Chotokukurshinga & $\mathrm{Fl}, \mathrm{Gl}, \mathrm{Ml}$ & Herb, er; w & Native $M A R 74$ & M \\
\hline Calendula officinalis L. + & Calendula & Gr, Rs & Herb, er; pl & Exotic SSS 2201 & $\mathrm{O}$ \\
\hline Cosmos bipinnatus Cav. & Cosmos & Gr, Rs & Herb, er; pl & Exotic GMH 5199 & $\mathrm{O}$ \\
\hline C. sulphureus Cav. & Cosmos & Gr, Rs & Herb, er; pl & Exotic SSS 2202 & $\mathrm{O}$ \\
\hline $\begin{array}{l}\text { Cotula hemisphaerica Wall. ex Benth. \& } \\
\text { Hook. f. }\end{array}$ & Nooney shak & $\mathrm{Gr}$ & Herb, pr; w & Native $S A K 2013$ & M \\
\hline Centipeda minima (L.) A. Br. \& Asch. & Nakchikni & $\mathrm{Fl}, \mathrm{Gl}, \mathrm{Lb}$ & Herb, pr; w & Native $M A R 78$ & M \\
\hline $\begin{array}{l}\text { Chromolaena odorata (L.) R.M. King \& } \\
\text { H. Rob. }\end{array}$ & Bon motmotia & $\mathrm{Bw}^{1}, \mathrm{Gl}, \mathrm{Lb}$ & Herb, er; w & Exotic SAK 2006 & M \\
\hline Cyanthillium cinereum (L.) H. Rob. & Shialmutra & $\mathrm{Bw}^{1}, \mathrm{Fl}, \mathrm{Gl}, \mathrm{Rs}$ & Herb, er; w & Native $M A R 145$ & M \\
\hline Dahlia imperialis Roezl ex Ortgies & Dalia & Gr, Rs & Herb, er; pl & Exotic SSS 2203 & $\mathrm{O}$ \\
\hline Eclipta prostrata (L.) L. & Kalokeshi & $\mathrm{Fl}, \mathrm{Gl}, \mathrm{Rs}$ & Herb, pr; w & Exotic SS 182 & M \\
\hline Elephantopus mollis Kunth & Hastipadi & Gl, Lb, Rs, Wl & Herb, cr; w & Exotic $S S 366$ & M \\
\hline Emilia sonchifolia (L.) DC. & Mechitra & $\mathrm{Fl}, \mathrm{Gl}, \mathrm{Lb}$ & Herb, er; w & Native $S S 367$ & M \\
\hline Enydra fluctuans DC. & Helencha & Wtl & Herb, pr; w & Native $S A K 2091$ & $\mathrm{M}, \mathrm{Vg}$ \\
\hline Gazania rigens (L.) Gaertn. + & Gaznia & Gr, Rs & Herb, er; pl & Exotic SSS 2204 & $\mathrm{O}$ \\
\hline Gerbera jamesonii Adlam + & Jarbera & Gr, Rs & Herb, er; pl & Exotic GMH 5159 & $\mathrm{O}$ \\
\hline Glebionis coronaria (L.) Cass. ex Spach & Chandramallika & Gr, Rs & Herb, er; pl & Exotic MAR 2731 & $\mathrm{O}$ \\
\hline Gnaphalium polycaulon Pers. & Bara kamra & $\mathrm{Fl}, \mathrm{Gr}, \mathrm{Gl}$ & Herb, er; w & Native MAR 172 & M \\
\hline Grangea maderaspatana (L.) Poir. & Namuti & $\mathrm{Fl}, \mathrm{Lb}$ & Herb, er; w & Native $S S 231$ & M \\
\hline Gynura procumbens (Lour.) Merr. ++ & Gynura & $\mathrm{Gr}, \mathrm{Hs}$ & Herb, er; pl-w & Native $S A K 2073$ & M \\
\hline
\end{tabular}


Table 1 contd.

\begin{tabular}{|c|c|c|c|c|c|}
\hline Scientific name & Bangla name & Habitat & Habit & Origin RSE & Use \\
\hline$\overline{\text { Launaea asplenifolia Hook.f. }}$ & Tik-chana & Fl, Rs & Herb, er; w & Exotic $S A K 2060$ & $\mathrm{M}$ \\
\hline Mikania cordata (Burm.f.) B.L. Rob. & Assam lata & $\mathrm{Fl}, \mathrm{Ml}, \mathrm{Sj}$ & Herb, vi; w & Exotic $S A K 2063$ & $\mathrm{Fd}, \mathrm{M}$ \\
\hline Parthenium hysterophorus L. + & Parthenum & $\mathrm{Fl}, \mathrm{Rs}$ & Herb, er; w & Exotic $S A K 2074$ & M, Po \\
\hline $\begin{array}{l}\text { Pseudognaphalium luteoalbum (L.) } \\
\text { Hilliard \& B.L. Burtt }\end{array}$ & Barakamra & $\mathrm{Fl}, \mathrm{Gl}, \mathrm{Rs}$ & Herb, er; w & Native $S S 183$ & M \\
\hline $\begin{array}{l}\text { Pseudelephantopus spicatus (B. Juss. ex } \\
\text { Aubl.) C.F. Baker }+\end{array}$ & Unknown & Ml, Rs & Herb, er; w & Exotic MAR 97 & M \\
\hline Sonchus arvensis $\mathrm{L}$. & Chashar & $\mathrm{Fl}, \mathrm{Gl}, \mathrm{Rs}$ & Herb, er; w & Exotic $S A K 2038$ & M \\
\hline Sphagneticola trilobata (L.) Pruski + & Mahabhringaraj & $\mathrm{Fl}, \mathrm{Rs}$ & Herb, pr; w & Exotic SS 214 & $\mathrm{Fd}, \mathrm{O}$ \\
\hline $\begin{array}{l}\text { Symphyotrichum laeve (L.) Á. Löve \& } \\
\text { D. Löve + }\end{array}$ & Aster & Gr, Rs & Herb, er; pl & Exotic $M A R 2732$ & $\mathrm{O}$ \\
\hline Synedrella nodiflora (L.) Gaertn. & Nak phul & $\mathrm{Fl}, \mathrm{Gl}, \mathrm{Rs}$ & Herb, er; w & Exotic SS 125 & M \\
\hline Tagetes erecta $\mathrm{L}$. & Gada, ganda & Herb, er; pl & Herb, er; pl & Exotic $G M H 5181$ & $\mathrm{M}, \mathrm{O}$ \\
\hline Tridax procumbens (L.) L. & Tridhara & $\mathrm{Bw}^{1}, \mathrm{Fl}, \mathrm{Rs}, \mathrm{Gl}$ & Herb, er; w & Exotic SS 181 & M \\
\hline Xanthium strumarium L. & Ghagra & $\mathrm{Fl}, \mathrm{Lb}, \mathrm{Gl}$ & Herb, er; w & Exotic MAR 103 & M \\
\hline Youngia japonica (L.) DC. & Youngaful & $\mathrm{Fl}, \mathrm{Gl}, \mathrm{Rs}$ & Herb, er; w & Native MAR 131 & M \\
\hline Zinnia peruviana $($ L.) L. + & Zinia & Gr, Rs & Herb, er; pl & Exotic SSS 2205 & $\mathrm{O}$ \\
\hline \multicolumn{6}{|l|}{ LILIOPSIDA Batsch } \\
\hline \multicolumn{6}{|l|}{ Alismataceae Vent. } \\
\hline Limnocharis flava (L.) Buchenau & Letuce pana & Wtl & Herb, er; w & Exotic GMH 5109 & Ap, $\mathrm{Vg}$ \\
\hline Sagittaria sagittifolia $\mathrm{L}$. & Chotokut & $\mathrm{Lb}, \mathrm{Wtl}$ & Herb, er; w & Exotic GMH 5110 & Fo, $\mathrm{O}$ \\
\hline \multicolumn{6}{|l|}{ Hydrocharitaceae Juss. } \\
\hline $\begin{array}{l}\text { Blyxa japonica (Miq.) Maxim. ex Asch. } \\
\& \text { Gürke }\end{array}$ & Japani blyxa & Lb, Wtl & Herb, sm; w & Native $M A R 2658$ & Fo \\
\hline Hydrilla verticillata (L. f.) Royle & Kureli & Wtl & Herb, sm; w & Native SS 409 & Ap, Ff \\
\hline Najas graminea Delile & Dhanijhaji & Wtl & Herb, sm; w & Native MAR 149 & $\mathrm{Nk}$ \\
\hline N. indica (Willd.) Cham. & Deshijhaji & Wtl & Herb, sm; w & Native $M A R 163$ & Ap \\
\hline N. minor All. & Soto jhaji & Wtl & Herb, sm; w & Native MAR 2659 & $\mathrm{Ff}$ \\
\hline $\begin{array}{l}\text { Nechamandra alternifolia (Roxb. ex } \\
\text { Wight) Thwaites }\end{array}$ & Rasna-zanji & Wtl & Herb, sm; w & Native $M A R 2660$ & $\mathrm{Nk}$ \\
\hline Ottelia alismoides (L.) Pers. & Panikala & Wtl & Herb, sm; w & Native $M A R 150$ & $\mathrm{Vg}$ \\
\hline Vallisneria spiralis $\mathrm{L}$. & Patseola & Wtl & Herb, sm; w & Native MAR 148 & Ap \\
\hline \multicolumn{6}{|l|}{ Aponogetonaceae Planch. } \\
\hline Aponogeton appendiculatus $\mathrm{H}$. Bruggen & Ghechu & Wtl & Herb, er; w & Native SSS 2165 & $\mathrm{Vg}$ \\
\hline A. undulatus Roxb. & Dheu-ghechu & Wtl & Herb, er; w & Native SSS 2166 & $\mathrm{Vg}$ \\
\hline \multicolumn{6}{|l|}{ Potamogetonaceae Bercht. \& J. Presl } \\
\hline Potamogeton crispus $\mathrm{L}$. & Pata zhanchi & Lb, Wtl & Herb, sm; w & Native SSS 2094 & $\mathrm{Vg}$ \\
\hline P. nodosus Poir. & Lombu zhanchi & $\mathrm{Lb}, \mathrm{Wtl}$ & Herb, sm; w & Native SSS 2095 & $\mathrm{Nk}$ \\
\hline \multicolumn{6}{|l|}{ Arecaceae Bercht. \& J. Presl } \\
\hline Areca catechu $\mathrm{L}$. & Supari & Gr, Hs, Rs & Palm, $1 ; \mathrm{pl}$ & Exotic SS 500 & Ed, M \\
\hline Attalea cohune Mart. & Cohune palm & $\mathrm{Gr}$ & Palm, $1 ; \mathrm{pl}$ & Exotic $M A R 2661$ & $\mathrm{O}$ \\
\hline Borassus flabellifer $\mathrm{L}$. & Tal & Gr, Hs, Rs & Palm, 1;pl-w & Native SS 501 & $\mathrm{Fb}, \mathrm{Ju}$ \\
\hline Calamus erectus Roxb. \# & Kdom bet & $\mathrm{Gr}, \mathrm{Lb}, \mathrm{Rs}$ & Palm, er; pl-w & Native $M A R 2662$ & $\mathrm{Cc}$ \\
\hline C. guruba Buch.-Ham. ex Mart. \# & Jali bet & $\mathrm{Gr}, \mathrm{Lb}, \mathrm{Sj}, \mathrm{Rs}$ & Palm, cl; pl-w & Native MAR 182 & $\mathrm{Cc}$ \\
\hline C. latifolius Roxb. \# & Sanchi bet & $\mathrm{Gr}, \mathrm{Lb}, \mathrm{Rs}$ & Palm, cl; pl-w & Native MAR 2663 & $\mathrm{Cc}$ \\
\hline C. longisetus Griff. \# & Karak bet & $\mathrm{Gr}, \mathrm{Lb}$ & Palm, cl; pl-w & Native $M A R 2664$ & $\mathrm{Cc}$ \\
\hline
\end{tabular}


Table 1 contd.

\begin{tabular}{|c|c|c|c|c|c|}
\hline Scientific name & Bangla name & Habitat & Habit & Origin RSE & Use \\
\hline C. tenuis Roxb. & Unknown & $\mathrm{Gr}, \mathrm{Lb}$ & Palm, cl; pl-w & Native $M A R 2665$ & $\mathrm{Cc}, \mathrm{Fr}$ \\
\hline Caryota urens $\mathrm{L}$. & Sago palm & Gr, Rs & Palm, 1; pl & Exotic SSS 2096 & $\mathrm{O}$ \\
\hline Chamaedorea elegans Mart. & Supari palm & $\mathrm{Gr}$ & Palm, s; pl & Exotic SSS 2097 & $\mathrm{O}$ \\
\hline Cocos nucifera $\mathrm{L}$. & Narikel & $\mathrm{Gr}, \mathrm{Hs}, \mathrm{Ml}$ & Palm, $1 ; \quad$ pl-w & Exotic SS 502 & Fw, Oy \\
\hline Corypha taliera Roxb.\# & Tali & Gr & Palm, 1; pl & Native $G M H 5100$ & $\mathrm{O}$ \\
\hline $\begin{array}{l}\text { Dypsis lutescens (H.Wendl.) Beentje \& } \\
\text { J. Dransf. }\end{array}$ & Areca palm & $\mathrm{Bw}^{2}, \mathrm{Gr}$ & Palm, cl; pl & Exotic MAR 2666 & $\mathrm{Cc}, \mathrm{O}$ \\
\hline Elaeis guineensis Jacq. & Oil palm & $\mathrm{Gr}, \mathrm{Hs}$ & Palm, 1; pl & Exotic GMH 5101 & Oy \\
\hline Licuala grandis $\mathrm{H}$. Wendl. & Vanuatu palm & $\mathrm{Gr}$ & Palm, $1 ; \mathrm{pl}$ & Exotic MAR 2667 & $\mathrm{O}$ \\
\hline L. peltata Roxb. ex Buch.-Ham. & Kurkuti & $\mathrm{Gr}$ & Palm, m; pl & Native $G M H 5102$ & $\mathrm{O}$ \\
\hline L. spinosa Wurmb & Katalicu & $\mathrm{Gr}$ & Palm, m; pl & Native MAR 2809 & $\mathrm{O}$ \\
\hline Livistona chinensis (Jacq.) R. Br. ex Mart. & China tokopata & $\mathrm{Gr}$ & Palm, m; pl & Exotic GMH 5103 & $\mathrm{O}$ \\
\hline Phoenix acaulis Roxb. \# & Khudi khejur & $\mathrm{Gr}$ & Palm, s; pl & Native GMH 5104 & $\mathrm{O}$ \\
\hline P. canariensis Chabaud & Canarian khejur & $\mathrm{Gr}$ & Palm, s; pl & Exotic $M A R 2728$ & $\mathrm{O}$ \\
\hline P. loureiroi Kunth & Bon khejur & $\mathrm{Sj}, \mathrm{Wl}$ & Palm, s; w & Native GMH 5105 & $\mathrm{O}$ \\
\hline P. sylvestris (L.) Roxb. & Deshi khejur & Hs, Ml, Rs & Palm, $1 ; \quad$ pl-w & Native $S S 408$ & $\mathrm{Hc}, \mathrm{Ju}$ \\
\hline Rhapis excelsa (Thunb.) Henry & Gurital & Gr & Palm, s; pl & Exotic SSS 2098 & $\mathrm{O}$ \\
\hline Roystonea regia (Kunth) O.F. Cook & Bottol palm & $\mathrm{Gr}$ & Palm, 1; pl & Exotic SSS 2099 & $\mathrm{O}$ \\
\hline \multicolumn{6}{|l|}{ Pandanaceae R. Br. } \\
\hline Pandanus amaryllifolius Roxb. & Polau pata & $\mathrm{Gr}, \mathrm{Hs}$ & Shrub; pl & Exotic SSS 2100 & $\mathrm{Ff}$ \\
\hline P. furcatus Roxb. & Kasiakata & $\mathrm{Gr}$ & Tree, s; pl & Native $M A R 2668$ & $\mathrm{O}, \mathrm{M}$ \\
\hline P. odorifer (Forssk.) Kuntze & Keya & $\mathrm{Gr}$ & Tree, s; pl & Native MAR 2669 & $\mathrm{Fb}, \mathrm{M}$ \\
\hline P. tectorius Parkinson ex Du Roi & Keuri kanta & $\mathrm{Gr}$ & Tree, s, pl & Exotic MAR 2670 & $\mathrm{Fb}, \mathrm{Ff}$ \\
\hline \multicolumn{6}{|l|}{ Araceae Juss. } \\
\hline Alocasia fornicata (Roxb.) Schott & Bish kachu & $\mathrm{Fl}, \mathrm{Hs}, \mathrm{Wtl}$ & Herb, er; w & Native $M A R 2671$ & M \\
\hline A. macrorrhizos (L.) G. Don & Man kachu & $\mathrm{Fl}, \mathrm{Hs}$ & Herb, er; pl-w & Exotic MAR 2672 & $\mathrm{M}, \mathrm{Vg}$ \\
\hline $\begin{array}{l}\text { Amorphophallus paeoniifolius (Dennst.) } \\
\text { Nicolson }\end{array}$ & Ol kachu & $\mathrm{Fl}, \mathrm{Hs}$ & Herb, er; pl-w & Native $M A R 2673$ & $\mathrm{M}, \mathrm{Vg}$ \\
\hline Anthurium andraeanum Linden ex André & Flamingo & Gr & Herb, er; pl & Exotic SSS 2142 & $\mathrm{O}$ \\
\hline A. crystallinum Linden \& André & Anthurium & $\mathrm{Gr}, \mathrm{Hs}$ & Herb, er; pl & Exotic SSS 2181 & $\mathrm{O}$ \\
\hline Caladium bicolor (Aiton) Vent. & Diranga kachu & $\mathrm{Gr}, \mathrm{Hs}$ & Herb, er; pl-w & Exotic MAR 2674 & $\mathrm{O}$ \\
\hline C. humboldtii (Raf.) Schott & Caladium & $\mathrm{Gr}, \mathrm{Hs}$ & Herb, er; pl & Exotic MAR 2816 & $\mathrm{O}$ \\
\hline Colocasia esculenta (L.) Schott & Jangli kachu & $\mathrm{Fl}, \mathrm{Hs}, \mathrm{Wtl}$ & Herb, er; w & Native SS 503 & $\mathrm{M}, \mathrm{Vg}$ \\
\hline Dieffenbachia seguine (Jacq.) Schott & Segu bet & $\mathrm{Fl}, \mathrm{Gr}, \mathrm{Hs}$ & Herb, er; pl-w & Exotic MAR 2817 & $\mathrm{O}$ \\
\hline $\begin{array}{l}\text { Epipremnum aureum (Linden \& André) } \\
\text { G.S. Bunting }\end{array}$ & Money plant & $\mathrm{Gr}, \mathrm{Hs}$ & Herb, vi; pl-w & Exotic SSS 2101 & $\mathrm{O}$ \\
\hline E. pinnatum (L.) Engl. & Premnum & $\mathrm{Gr}, \mathrm{Hs}$ & Herb, vi; pl-w & Native SSS 2102 & $\mathrm{M}, \mathrm{O}$ \\
\hline Lasia spinosa $(\mathrm{L}$.$) Thwaites$ & Kata kachu & $\mathrm{Hs}, \mathrm{Lb}, \mathrm{Wtl}$ & Herb, er; w & Native SSS 2103 & $\mathrm{M}, \mathrm{Vg}$ \\
\hline Lemna minor $\mathrm{L}$. & Sujipana & Wtl & Herb, ff; w & Native SSS 2104 & Ff, Wp \\
\hline L. perpusilla Torr. & Khudipana & Wtl & Herb, ff; w & Exotic SSS 2105 & Ef, Wp \\
\hline Monstera deliciosa Liebm. & Makhna & $\mathrm{Gr}, \mathrm{Hs}$ & Herb, vi; pl-w & Exotic $S S 407$ & Ed, O \\
\hline Pistia stratiotes L. & Topapana & Wtl & Herb, ff; w & Native SS 504 & M \\
\hline Pothos scandens $\mathrm{L}$. & Hati lata & Op & Herb, cr; pl-w & Native $S S 505$ & M \\
\hline Scindapsus officinalis (Roxb.) Schott & Gaj-pipul & Op & Herb, vi; pl-w & Native SSS 2182 & M \\
\hline Spirodela polyrhiza (L.) Schleid. & Tetulipana & Wtl & Herb, ff; w & Native $M A R 152$ & Ff, Wp \\
\hline Syngonium podophyllum Schott & Podolata kachu & $\mathrm{Fl}, \mathrm{Gr}, \mathrm{Hs}, \mathrm{Sj}$ & Herb, pr; pl-w & Native SSS 2183 & $\mathrm{O}$ \\
\hline
\end{tabular}


Table 1 contd.

\begin{tabular}{|c|c|c|c|c|c|}
\hline Scientific name & Bangla name & Habitat & Habit & Origin RSE & Use \\
\hline Typhonium flagelliforme (Lodd.) Blume & Ghechu & $\mathrm{Fl}, \mathrm{Lb}, \mathrm{Rs}$ & Herb, er; w & Native $G M H 5106$ & $\mathrm{M}$ \\
\hline T. trilobatum (L.) Schott & Ghet kachu & $\mathrm{Fl}, \mathrm{Lb}, \mathrm{Rs}$ & Herb, er; w & Native GMH 5107 & $\mathrm{M}, \mathrm{Vg}$ \\
\hline Wolffia arrhiza (L.) Horkel ex Wimm. & Guripana & Wtl & Herb, ff; w & Native SSS 2184 & Ff, Wp \\
\hline Xanthosoma sagittifolium (L.) Schott & Dudh kachu & $\mathrm{Fl}, \mathrm{Lb}, \mathrm{Wtl}$ & Herb, er; w & Exotic GMH 5108 & $\mathrm{M}, \mathrm{Vg}$ \\
\hline \multicolumn{6}{|l|}{ Commelinaceae Mirb. } \\
\hline Commelina benghalensis $\mathrm{L}$. & Kanshira & Fl, Gl, Lb, Rs & Herb, cr; w & Native SS 229 & M \\
\hline C. diffusa Burm.f. & Monayna kanshira & $\mathrm{Fl}, \mathrm{Gl}, \mathrm{Lb}, \mathrm{Rs}$ & Herb, cr; w & Native SS 506 & Wd \\
\hline C. erecta $\mathrm{L}$. & Jata kanchira & $\mathrm{Fl}, \mathrm{Gl}, \mathrm{Lb}, \mathrm{Rs}$ & Herb, er; w & Exotic MAR 154 & $\mathrm{Wd}$ \\
\hline C. longifolia Lam. & Pani kanshira & $\mathrm{Fl}, \mathrm{Gl}, \mathrm{Lb}, \mathrm{Rs}$ & Herb, cr; w & Native MAR 104 & Wd \\
\hline C. paludosa Blume & Kanjia & $\mathrm{Fl}, \mathrm{Gl}, \mathrm{Lb}, \mathrm{Rs}$ & Herb, er; w & Native $M A R 153$ & Wd \\
\hline Cyanotis axillaris (L.) D. Don ex Sweet & Axinot & $\mathrm{Fl}, \mathrm{Gl}, \mathrm{Rs}$ & Herb, er; w & Native GMH 5111 & Wd \\
\hline C. cristata (L.) D. Don. & Tata- kansira & $\mathrm{Fl}, \mathrm{Gl}, \mathrm{Lb}$ & Herb, cr; w & Native SS 295 & M \\
\hline Murdannia nudiflora (L.) Brenan & Kureli & $\mathrm{Fl}, \mathrm{Lb}, \mathrm{Ml}$ & Herb, cr; w & Native MAR 155 & $\mathrm{Wd}$ \\
\hline Tradescantia pallida (Rose) D.R. Hunt & Begunipindo & $\mathrm{Gr}$ & Herb, er; pl-w & Exotic SSS 2106 & $\mathrm{O}$ \\
\hline T. spathacea $\mathrm{Sw}$. & Chamapindo & Gr & Herb, er; pl-w & Exotic SSS 2107 & $\mathrm{O}$ \\
\hline T. zebrina Bosse & Zebrapindo & Gr & Herb, er; pl-w & Exotic SSS 2108 & $\mathrm{O}$ \\
\hline \multicolumn{6}{|l|}{ Eriocaulaceae Martinov } \\
\hline Eriocaulon quinquangulare $\mathrm{L}$. & Guriguccha & $\mathrm{Lb}, \mathrm{Wtl}$ & Herb, er; w & Native SS 204 & Wd \\
\hline \multicolumn{5}{|l|}{ Cyperaceae Juss. } & Wd \\
\hline Cyperus compressus $\mathrm{L}$. & Chancha & $\mathrm{Fl}, \mathrm{Gl}, \mathrm{Rs}$ & Herb, er; w & Native SSS 2110 & $\mathrm{Wd}$ \\
\hline C. corymbosus Rottb. & Golamethi & $\mathrm{Fl}, \mathrm{Gl}, \mathrm{Lb}, \mathrm{Rs}$ & Herb, er; w & Native SS 206 & $\mathrm{Fb}, \mathrm{Fo}$ \\
\hline C. cuspidatus Kunth & Sagarmukhimethi & $\mathrm{Fl}, \mathrm{Gl}, \mathrm{Rs}$ & Herb, er; w & Native MAR 156 & $\mathrm{Wd}$ \\
\hline C. cyperoides (L.) Kuntze & Kucha & $\mathrm{Fl}, \mathrm{Gl}, \mathrm{Rs}$ & Herb, er; w & Native SS 298 & $\mathrm{Ed}$ \\
\hline C. difformis $\mathrm{L}$. & Behua ghasi & $\mathrm{Fl}, \mathrm{Gl}, \mathrm{Lb}$ & Herb, er; w & Native SSS 2111 & Fo, Wd \\
\hline C. digitatus Roxb. & Hath ghasi & Gl, Lb, Wtl & Herb, er; w & Native SSS 2112 & $\mathrm{Wd}$ \\
\hline C. distans $\mathrm{L} . \mathrm{f}$. & Panimalanga & $\mathrm{Bw}^{1}, \mathrm{Fl}, \mathrm{Gl}, \mathrm{Rs}$ & Herb, er; w & Native SS207 & Fo \\
\hline C. exaltatus Retz. & Tata ghasi & $\mathrm{Gl}, \mathrm{Lb}, \mathrm{Wtl}$ & Herb, er; w & Native $G M H 5112$ & $\mathrm{Fb}, \mathrm{Wd}$ \\
\hline C. haspan $\mathrm{L}$. & Haspan ghasi & $\mathrm{Fl}, \mathrm{Gl}, \mathrm{Wtl}$ & Herb, er; w & Native $M A R 2675$ & $\mathrm{Wd}$ \\
\hline C. iria $\mathrm{L}$. & Barachucha & $\mathrm{Fl}, \mathrm{Gl}, \mathrm{Rs}$ & Herb, er; w & Native $M A R 2676$ & $\mathrm{Fb}, \mathrm{M}$ \\
\hline C. pilosus Vahl & Pashamkathai & $\mathrm{Fl}, \mathrm{Gl}, \mathrm{Lb}$ & Herb, er; w & Native $M A R 118$ & Wd \\
\hline C. pulcherrimus Willd. ex Kunth & Unknown & $\mathrm{Gl}, \mathrm{Lb}, \mathrm{Wtl}$ & Herb, er; w & Native MAR 2677 & $\mathrm{Wd}$ \\
\hline C. rotundus $\mathrm{L}$. & Nagarmutha & Fl, Gl, Hs, Rs & Herb, er; w & Native SSS 2113 & M \\
\hline C. substramineus Kük. & Paikram ghas & Fl, Gl, Lb, Rs & Herb, er; w & Native SS 213 & $\mathrm{Wd}$ \\
\hline C. tenuispica Steud. & Paikamutha & $\mathrm{Gl}, \mathrm{Lb}, \mathrm{Wtl}$ & Herb, er; w & Native SS 302 & $\mathrm{Sb}, \mathrm{Wd}$ \\
\hline C. thomsonii Boeckeler \# & Sanimutha & $\mathrm{Fl}, \mathrm{Gl}, \mathrm{Rs}$ & Herb, er; w & Native MAR 119 & Wd \\
\hline Diplacrum caricinum $\mathrm{R}$. Br. & Unknown & $\mathrm{Fl}, \mathrm{Lb}, \mathrm{Rs}$ & Herb, pr; w & Native $M A R 2678$ & Wd \\
\hline Eleocharis acutangula (Roxb.) Schult. & Unknown & $\mathrm{Lb}, \mathrm{Ml}, \mathrm{Wtl}$ & Herb, er; w & Native SSS 2114 & $\mathrm{Sb}, \mathrm{Wd}$ \\
\hline E. dulcis (Burm. f.) Trin. ex Hensch. & Mishti ghasi & $\mathrm{Fl}, \mathrm{Lb}, \mathrm{Ml}$ & Herb, er; w & Native SSS 2115 & Ed \\
\hline $\begin{array}{l}\text { Fimbristylis bisumbellata (Forssk.) } \\
\text { Bubani }\end{array}$ & Bisu fimbry & $\mathrm{Lb}, \mathrm{Ml}, \mathrm{Wtl}$ & Herb, er; w & Native SSS 2116 & $\mathrm{Wd}$ \\
\hline F. dichotoma (L.) Vahl & Bara nirbishi & $\mathrm{Bw}^{1}, \mathrm{Fl}, \mathrm{Gl}, \mathrm{Rs}$ & Herb, er; w & Native GMH 5113 & Wd \\
\hline F. ovata (Burm. f.) J. Kern & Marmari & $\mathrm{Lb}, \mathrm{Ml}, \mathrm{Wtl}$ & Herb, er; w & Native MAR106 & $\mathrm{Wd}$ \\
\hline F. quinquangularis (Vahl) Kunth & Barajavani & $\mathrm{Fl}, \mathrm{Lb}, \mathrm{Wtl}$ & Herb, er; w & Native $M A R 2679$ & Wd \\
\hline F. schoenoides (Retz.) Vahl & Kesari malanga & $\mathrm{Fl}, \mathrm{Lb}, \mathrm{Wtl}$ & Herb, er; w & Native SSS 2117 & $\mathrm{Wd}$ \\
\hline
\end{tabular}


Table 1 contd.

\begin{tabular}{|c|c|c|c|c|c|}
\hline Scientific name & Bangla name & Habitat & Habit & Origin RSE & Use \\
\hline F. tetragona $\mathrm{R} . \mathrm{Br}$. & Unknown & Gl, Lb, Wtl & Herb, er; w & Native $\operatorname{SSS} 2118$ & $\mathrm{Wd}$ \\
\hline Fuirena ciliaris (L.) Roxb. & Poshmi ghas & Gl, Lb, Wtl & Herb, er; w & Native $S S 212$ & Wd \\
\hline F. umbellata Rottb. & Unknown & Gl, Lb, Wtl & Herb, er; w & Native SSS 2119 & $\mathrm{Ed}, \mathrm{Wd}$ \\
\hline Kyllinga brevifolia Rottb. & Shabujnirbisa & $\mathrm{Fl}, \mathrm{Gl}, \mathrm{Lb}, \mathrm{Rs}$ & Herb, er; w & Native SS 305 & Fo \\
\hline $\begin{array}{l}\text { K. nemoralis (J.R. Forst. \& G. Forst.) } \\
\text { Dandy ex Hutch. \& Dalziel }\end{array}$ & Subashinirbisa & $\mathrm{Fl}, \mathrm{Gl}, \mathrm{Lb}, \mathrm{Rs}$ & Herb, er; w & Native SSS 2120 & Ed, Fo \\
\hline Pycreus pumilus (L.) Nees & Paikpami ghasi & $\mathrm{Fl}, \mathrm{Gl}, \mathrm{Lb}, \mathrm{Rs}$ & Herb, er; w & Native $M A R 2680$ & $\mathrm{Sb}, \mathrm{Wd}$ \\
\hline Schoenoplectiella articulata (L.) Lye & Chechra & $\mathrm{Fl}, \mathrm{Lb}, \mathrm{Rs}$ & Herb, er; w & Native $G M H 5114$ & $\mathrm{Wd}$ \\
\hline S. juncoides (Roxb.) Lye & Chechri & $\mathrm{Fl}, \mathrm{Lb}, \mathrm{Rs}$ & Herb, er; w & Native SSS 2121 & $\mathrm{Wd}$ \\
\hline S. supina (L.) Lye & Supipotpoti ghas & $\mathrm{Fl}, \mathrm{Lb}, \mathrm{Rs}$ & Herb, er; w & Native MAR 157 & $\mathrm{Wd}$ \\
\hline Scleria levis Retz. & Rialevi ghas & $\mathrm{Fl}, \mathrm{Lb}, \mathrm{Rs}$ & Herb, cr; w & Native SS309 & Wd \\
\hline \multicolumn{6}{|l|}{ Poaceae Barnhart } \\
\hline Alloteropsis cimicina $(\mathrm{L}$.$) Stapf$ & Unknown & Gl, Rs & Herb, er; w & Native SSS 2122 & Wd \\
\hline Apluda mutica $\mathrm{L}$. & Matika & $\mathrm{Lb}, \mathrm{Sj}$ & Herb, er; w & Native $M A R 2681$ & Fo \\
\hline Arundinella bengalensis (Spreng) Druce & Gangabena & $\mathrm{Gl}, \mathrm{Lb}$ & Herb, er; w & Native $S S 174$ & Wd \\
\hline Axonopus compressus (Sw.) P. Beauv. & Karpet ghas & $\mathrm{Bw}^{1}, \mathrm{Gl}, \mathrm{Rs}$ & Herb, er; w & Exotic $S S 412$ & Fo \\
\hline Bambusa balcooa Roxb. & Borak bans & $\mathrm{Gr}, \mathrm{Hs}$ & Bamboo; pl & Native SSS 2123 & $\mathrm{Bc}$ \\
\hline B. bambos (L.) Voss & Kanta bans & Gr & Bamboo; pl & Native $M A R 181$ & $\mathrm{Bc}$ \\
\hline B. multiplex (Lour.) Raeusch. ex Schult. & Choi bans & Gr & Bamboo; pl & Exotic $M A R 2682$ & $\mathrm{Bc}$ \\
\hline B. nutans Wall. ex Munro & Mahal bans & $\mathrm{Gr}, \mathrm{Hs}$ & Bamboo; pl & Native $M A R 2683$ & $\mathrm{Bc}$ \\
\hline B. polymorpha Munro & Parua & Gr & Bamboo; pl & Native SSS 2124 & $\mathrm{Bc}$ \\
\hline B. salarkhanii Alam & Jaotha bans & Gr & Bamboo; pl & Native SSS 2125 & $\mathrm{Bc}$ \\
\hline B. tulda Roxb. & Mirtinga & $\mathrm{Gr}, \mathrm{Hs}$ & Bamboo; pl & Native GMH 5115 & $\mathrm{Bc}$ \\
\hline B. tuldoides Munro & Ghoti bans & $\mathrm{Gr}, \mathrm{Hs}$ & Bamboo; pl & Exotic MAR 2683 & $\mathrm{Bc}$ \\
\hline B. vulgaris Schrad. & Bariala & Gr & Bamboo; pl & Exotic MAR 2684 & $\mathrm{Bc}$ \\
\hline B. mutica (Forssk.) Stapf & Para ghas & Gl, Rs & Herb, er; w & Exotic MAR 158 & $\mathrm{Fo}, \mathrm{Sb}$ \\
\hline $\begin{array}{l}\text { B. reptans (L.) C.A. Gardner \& C.E. } \\
\text { Hubb. }\end{array}$ & Unknown & $\mathrm{Fl}, \mathrm{Lb}$ & Herb, er; w & Native $M A R 2685$ & Fo \\
\hline Chrysopogon aciculatus (Retz.) Trin. & Prem kanta & Gl, Rs & Herb, er; w & Native $S S 314$ & $\mathrm{Hc}$ \\
\hline C. zizanioides (L.) Roberty & Bena & $\mathrm{Bw}^{1}, \mathrm{Fl}, \mathrm{Lb}$ & Herb, er; w & Native SSS 2128 & $\mathrm{Hc}, \mathrm{Sb}$ \\
\hline Chloris barbata $\mathrm{Sw}$. & Unknown & $\mathrm{Fl}, \mathrm{Gl}, \mathrm{Rs}$ & Herb, er; w & Native $M A R 2686$ & Fo \\
\hline C. virgata $\mathrm{Sw}$. & Anguli ghas & Gl, Rs & Herb, er; w & Native MAR 113 & Fo \\
\hline Coix lacryma-jobi $\mathrm{L}$. & Tasbi & $\mathrm{Lb}, \mathrm{Wtl}$ & Herb, er; w & Native SS 413 & Ed, Fo \\
\hline Cynodon dactylon (L.) Pers. & Durba ghas & $\mathrm{Fl}, \mathrm{Gl}, \mathrm{Rs}$ & Herb, pr; w & Native GMH 5116 & $\mathrm{Fo}, \mathrm{Sb}$ \\
\hline $\begin{array}{l}\text { Cyrtococcum oxyphyllum (Hochst. ex } \\
\text { Steud.) Stapf }\end{array}$ & Oxycocca ghas & $\mathrm{Fl}, \mathrm{Gl}, \mathrm{Rs}$ & Herb, er; w & Native SSS 2129 & Fo \\
\hline C. patens (L.) A. Camus & Unknown & $\mathrm{Fl}, \mathrm{Gl}, \mathrm{Rs}$ & Herb, er; w & Native SS 320 & Fo \\
\hline Cymbopogon citratus (DC.) Stapf & Lemon ghas & $\mathrm{Fl}, \mathrm{Ml}, \mathrm{Rs}$ & Herb, er; cv-w & Exotic SSS 2130 & Ff, M \\
\hline Dactyloctenium aegyptium (L.) Willd. & Kakpaya & $\mathrm{Fl}, \mathrm{Gl}, \mathrm{Rs}$ & Herb, er; w & Native GMH 5117 & $\mathrm{Sb}$ \\
\hline Dendrocalamus giganteus Munro & Budum bans & $\mathrm{Gr}$ & Bamboo; pl & Exotic $G M H 5118$ & $\mathrm{Bc}, \mathrm{O}$ \\
\hline D. longispathus (Kurz) Kurz & Orah bans & $\mathrm{Gr}$ & Bamboo; pl & Native SSS 2131 & $\mathrm{Bc}$ \\
\hline Desmostachya bipinnata (L.) Stapf & Kusha & $\mathrm{Fl}, \mathrm{Gl}, \mathrm{Lb}, \mathrm{Rs}$ & Herb, er; w & Native $M A R 2687$ & $\mathrm{Bc}$ \\
\hline Dichanthium annulatum (Forssk.) Stapf & Loari & $\mathrm{Bw}^{1}, \mathrm{Fl}, \mathrm{Gl}, \mathrm{Rs}$ & Herb, er; w & Native $M A R 2688$ & Fo \\
\hline D. caricosum (L.) A. Camus & Detara & $\mathrm{Fl}, \mathrm{Gl}, \mathrm{Rs}$ & Herb, er; w & Native $M A R 2689$ & Fo \\
\hline $\begin{array}{l}\text { Digitaria bicornis (Lam.) Roem. \& } \\
\text { Schult. }\end{array}$ & Baikochira & $\mathrm{Fl}, \mathrm{Gl}, \mathrm{Ml}, \mathrm{Rs}$ & Herb, er; w & Native SSS 2132 & Fo \\
\hline
\end{tabular}


Table 1 contd.

\begin{tabular}{|c|c|c|c|c|c|}
\hline Scientific name & Bangla name & Habitat & Habit & Origin RSE & Use \\
\hline D. ciliaris (Retz.) Koeler & Kokjachira & $\mathrm{Fl}, \mathrm{Gl}, \mathrm{Ml}, \mathrm{Rs}$ & Herb, er; w & Native SSS 2133 & Fo \\
\hline D. sanguinalis (L.) Scop. & Mukurjoli & $\mathrm{Fl}, \mathrm{Gl}, \mathrm{Ml}, \mathrm{Rs}$ & Herb, er; w & Native SSS 2134 & Fo \\
\hline D. setigera Roth & Seti ghas & $\mathrm{Bw}^{1}, \mathrm{Fl}, \mathrm{Gl}, \mathrm{Rs}$ & Herb, pr; w & Native MAR 2713 & Fo \\
\hline D. stricta Roth & Unknown & $\mathrm{Fl}, \mathrm{Gl}, \mathrm{Ml}, \mathrm{Rs}$ & Herb, er; w & Native $M A R 2690$ & Fo \\
\hline D. ternata (A. Rich.) Stapf & Unknown & $\mathrm{Fl}, \mathrm{Gl}, \mathrm{Ml}, \mathrm{Rs}$ & Herb, er; w & Native $M A R 2691$ & Fo \\
\hline Echinochloa colona (L.) Link. & Shama ghas & $\mathrm{Fl}, \mathrm{Ml}, \mathrm{Wtl}$ & Herb, er; w & Exotic MAR 121 & Fo \\
\hline E. crus-galli (L.) P. Beauv. & Barashama ghas & $\mathrm{Fl}, \mathrm{Ml}, \mathrm{Wtl}$ & Herb, er; w & Native $S S 414$ & Fo, M \\
\hline E. stagnina (Retz.) P. Beauv. & Parua & $\mathrm{Fl}, \mathrm{Ml}, \mathrm{Wtl}$ & Herb, er; w & Native SSS 2136 & $\mathrm{Fo}, \mathrm{Ju}$ \\
\hline Eleusine indica (L.) Gaertn. & Malankuri & $\mathrm{Bw}^{1}, \mathrm{Fl}, \mathrm{Gl}, \mathrm{Rs}$ & Herb, er; w & Native SSS 2137 & $\mathrm{Sb}$ \\
\hline Eragrostis unioloides (Retz.) Nees & Koni ghas & $\mathrm{Bw}^{1}, \mathrm{Fl}, \mathrm{Gl}, \mathrm{Rs}$ & Herb, er; w & Native MAR 2692 & Fo \\
\hline E. cilianensis (All.) Janch. & Dudh-nol & $\mathrm{Fl}, \mathrm{Gl}, \mathrm{Ml}, \mathrm{Rs}$ & Herb, er; w & Native $S S 415$ & Fo \\
\hline E. ciliaris (L.) R. Br. & Chhotochira ghas & $\mathrm{Fl}, \mathrm{Gl}, \mathrm{Ml}, \mathrm{Rs}$ & Herb, er; w & Native $M A R 2693$ & Ed, Fo \\
\hline E. unioloides (Retz.) Nees ex Steud. & Chirakoni & $\mathrm{Fl}, \mathrm{Gl}, \mathrm{Ml}, \mathrm{Rs}$ & Herb, er; w & Native SSS 2138 & $\mathrm{Gm}$ \\
\hline Hemarthria protensa Steud. & Chaila & $\mathrm{Lb}, \mathrm{Ml}, \mathrm{Wtl}$ & Herb, er; w & Native MAR 180 & $\mathrm{Nk}$ \\
\hline $\begin{array}{l}\text { Hygroryza aristata (Retz.) Nees ex } \\
\text { Wight \& Arn. }\end{array}$ & Jongli dhan & $\mathrm{Lb}, \mathrm{Ml}, \mathrm{Wtl}$ & Herb, er; w & Native GMH 5119 & Fo \\
\hline Hymenachne amplexicaulis (Rudge) Nees & Nordula ghas & Lb, Ml, Wtl & Herb, er; w & Exotic MAR 179 & Fo \\
\hline Isachne globosa (Thunb.) Kuntze & Ball ghas & $\mathrm{Fl}, \mathrm{Gl}, \mathrm{Wtl}$ & Herb, er; w & Native MAR 2694 & Fo, Gm \\
\hline Imperata cylindrica (L.) Raeusch. & Chhan & $\mathrm{Bw}^{1}, \mathrm{Fl}, \mathrm{Gl}, \mathrm{Rs}$ & Herb, er; w & Exotic $G M H 5120$ & $\mathrm{Nk}$ \\
\hline Leersia hexandra Sw. & Arali & $\mathrm{Fl}, \mathrm{Wtl}$ & Herb, cr; w & Native $G M H 5121$ & Fo \\
\hline Leptochloa chinensis (L.) Nees & Fulka ghas & $\mathrm{Fl}, \mathrm{Wtl}$ & Herb, er; w & Native $S S 208$ & Fo \\
\hline L. panicea (Retz.) Ohwi & Mona ghas & $\mathrm{Fl}, \mathrm{Wtl}$ & Herb, er; w & Native MAR 2695 & Fo \\
\hline Lophatherum gracile Brongn. & Lophail ghas & $\mathrm{Fl}, \mathrm{Gl}, \mathrm{Ml}, \mathrm{Rs}$ & Herb, er; w & Native $M A R 2696$ & Fo \\
\hline Melocanna baccifera (Roxb.) Kurz & Muli bans & $\mathrm{Gr}$ & Herb, er; w & Native SSS 2139 & $\mathrm{Bc}$ \\
\hline Oplismenus burmanni (Retz.) P. Beauv. & Jabri durba & $\mathrm{Fl}, \mathrm{Gl}, \mathrm{Ml}, \mathrm{Rs}$ & Herb, er; w & Native GMH 5122 & Fo \\
\hline O. compositus (L.) P. Beauv. & Gohur & $\mathrm{Gl}, \mathrm{Ml}, \mathrm{Rs}, \mathrm{Sj}$ & Herb, er; w & Native $S S 180$ & Fo \\
\hline Oryza rufipogon Griff. & Bunodhan & $\mathrm{Fl}, \mathrm{Wtl}$ & Herb, er; w & Native $G M H 5123$ & Fo \\
\hline O. sativa $\mathrm{L}$. & Dhan & $\mathrm{Fl}, \mathrm{Wtl}$ & Herb, er; cv & Exotic SSS 2140 & $\mathrm{Ed}, \mathrm{Fd}$ \\
\hline Ottochloa nodosa (Kunth) Dandy & Voyal ghas & $\mathrm{Fl}, \mathrm{Gl}, \mathrm{Ml}, \mathrm{Rs}$ & Herb, er; w & Native SSS 2141 & Fo \\
\hline Panicum brevifolium $\mathrm{L}$. & Bashpatighas & $\mathrm{Gl}, \mathrm{Lb}, \mathrm{Ml}$ & Herb, er; w & Native $S S 418$ & Fo \\
\hline P. incomtum Trin. & Panick ghas & $\mathrm{Fl}, \mathrm{Gl}, \mathrm{Lb}$ & Herb, er; w & Native MAR 2697 & Fo \\
\hline P. notatum Retz. & Panita ghas & $\mathrm{Fl}, \mathrm{Gl}, \mathrm{Ml}$ & Herb, er; w & Native $M A R 2698$ & Fo \\
\hline P. paludosum Roxb. & Borati & $\mathrm{Lb}, \mathrm{Ml}, \mathrm{Wtl}$ & Herb, er; w & Native $M A R 2699$ & Fo \\
\hline P. repens $\mathrm{L}$. & Dhani ghas & $\mathrm{Fl}, \mathrm{Gl}, \mathrm{Rs}$ & Herb, er; w & Native $S S 419$ & Fo \\
\hline P. sarmentosum Roxb. & Voya ghas & $\mathrm{Fl}, \mathrm{Gl}, \mathrm{Ml}, \mathrm{Rs}$ & Herb, er; w & Native MAR 2700 & Fo \\
\hline Paspalum conjugatum P.J. Bergius. & Moisshya ghas & Gl, Hs, Rs & Herb, er; w & Exotic $G M H 5124$ & $\mathrm{Fo}, \mathrm{Sb}$ \\
\hline P. distichum $\mathrm{L}$. & Chhotogoicha & $\mathrm{Lb}, \mathrm{Ml}, \mathrm{Wtl}$ & Herb, er; w & Exotic $M A R 178$ & $\mathrm{Fo}, \mathrm{Sb}$ \\
\hline P. scrobiculatum $\mathrm{L}$. & Bishmona ghas & $\mathrm{Fl}, \mathrm{Gl}, \mathrm{Rs}$ & Herb, er; w & Native $M A R 2701$ & Fo, M \\
\hline Pennisetum purpureum Schumach. & Nepi ghas & $\mathrm{Fl}, \mathrm{Gl}, \mathrm{Ml}, \mathrm{Rs}$ & Herb, er; cv-w & Exotic SSS 2143 & Fo \\
\hline $\begin{array}{l}\text { Phyllostachys aurea Rivière \& C. } \\
\text { Rivière }\end{array}$ & Sarna bans & $\mathrm{Fl}, \mathrm{Gl}, \mathrm{Ml}, \mathrm{Rs}$ & Bamboo; pl & Exotic SSS 2144 & $\mathrm{Bc}, \mathrm{O}$ \\
\hline Pseudoraphis brunoniana (Griff.) Pilg. & Rafi ghas & $\mathrm{Lb}, \mathrm{Wtl}$ & Herb, em; w & Native SSS 2145 & Fo \\
\hline $\begin{array}{l}\text { Rottboellia cochinchinensis (Lour.) } \\
\text { Clayton }\end{array}$ & Boro-sowati ghas & $\mathrm{Lb}, \mathrm{Wtl}$ & Herb, er; w & Native $G M H 5125$ & Fo \\
\hline Saccharum officinarum $\mathrm{L}$. & Akh & $\mathrm{Gr}, \mathrm{Hs}$ & Herb, er; cv & Exotic MAR 2702 & $\mathrm{Ju}, \mathrm{Pp}$ \\
\hline S. spontaneum $\mathrm{L}$. & Kash & $\mathrm{Fl}, \mathrm{Gl}, \mathrm{Ml}, \mathrm{Lb}$ & Herb, er; w & Native SSS 2146 & $\mathrm{Fo}, \mathrm{Sb}$ \\
\hline
\end{tabular}


Table 1 contd.

\begin{tabular}{|c|c|c|c|c|c|}
\hline Scientific name & Bangla name & Habitat & Habit & Origin RSE & Use \\
\hline Sacciolepis indica (L). A. Chase & Siltatto ghas & $\mathrm{Lb}, \mathrm{Ml}, \mathrm{Wtl}$ & Herb, er; w & Native SS 421 & Fo \\
\hline S. interrupta (Willd.) Stapf & Nardula & $\mathrm{Fl}, \mathrm{Gl}$ & Herb, er; w & Native $S S 422$ & Fo \\
\hline S. myosuroides (R. Br.) A. Camus & Musurdolla ghas & $\mathrm{Fl}, \mathrm{Gl}, \mathrm{Wtl}$ & Herb, er; w & Native $M A R 2703$ & Fo \\
\hline Setaria barbata (Lam.) Kunth & Bashpata ghas & $\mathrm{Fl}, \mathrm{Gl}, \mathrm{Ml}$ & Herb, er; w & Native MAR 382 & Fo \\
\hline S. flavida (Retz.) Veldkamp & Karin ghas & $\mathrm{Fl}, \mathrm{Gl}, \mathrm{Rs}$ & Herb, er; w & Native $S S 420 b$ & Fo \\
\hline S. palmifolia (J. Koenig) Stapf & Urodhan & $\mathrm{Fl}, \mathrm{Gl}, \mathrm{Ml}$ & Herb, er; w & Native $M A R 2704$ & Fo \\
\hline S. pumila (Poir.) Roem. \& Schult. & Halde kawn & $\mathrm{Fl}, \mathrm{Gl}, \mathrm{Ml}$ & Herb, er; w & Native $M A R 2705$ & Fo \\
\hline Sporobolus diandrus (Retz.) P. Beauv. & Benajoni & Fl, Gl, Ml, Rs & Herb, er; w & Native $S S 338$ & Fo \\
\hline S. indicus (L.) R. Br. & Ailbelajoni ghas & $\mathrm{Fl}, \mathrm{Gl}, \mathrm{Ml}, \mathrm{Rs}$ & Herb, er; w & Exotic $M A R 2706$ & Fo \\
\hline Thyrsostachys oliveri Gamble & Burma bans & $\mathrm{Fl}, \mathrm{Gl}, \mathrm{Ml}, \mathrm{Rs}$ & Herb, er; w & Exotic $M A R 2707$ & $\mathrm{Bc}$ \\
\hline $\begin{array}{l}\text { Thysanolaena latifolia (Roxb. ex } \\
\text { Hornem.) Honda ++ }\end{array}$ & Phul jharu & $\mathrm{Gr}, \mathrm{Hs}, \mathrm{Ml}$ & Herb, er; cv-w & Native $S S 340$ & Fo, $\mathrm{Hc}$ \\
\hline Urochloa distachyos (L.) T.Q. Nguyen, & Cori ghas & Gl, Rs & Herb, cr; w & Native SSS 2126 & $\mathrm{Fo}, \mathrm{Sb}$ \\
\hline U. kurzii (Hook.f.) T.Q. Nguyen, & Unknown & Gl, Lb, Rs & Herb, cr; w & Native SSS 2127 & Fo \\
\hline U. panicoides $\mathrm{P}$. Beauv. & Kuridana & $\mathrm{Fl}, \mathrm{Ml}$ & Herb, er; w & Native SSS 2147 & $\mathrm{Sb}$ \\
\hline Zea mays $\mathrm{L}$. & Bhutta & $\mathrm{Fl}, \mathrm{Hs}$ & Herb, er; cv & Exotic SSS 2148 & Ed \\
\hline \multicolumn{6}{|l|}{ Strelitziaceae Hutch. } \\
\hline Ravenala madagascariensis Sonn. & Panthopadap & Gr & Tree, s; pl & Exotic SSS 2149 & $\mathrm{O}$ \\
\hline Strelitzia reginae Banks & Behester bulbuli & Gr & Herb, er; pl & Exotic SSS 2150 & $\mathrm{O}$ \\
\hline \multicolumn{6}{|l|}{ Heliconiaceae Nakai } \\
\hline $\begin{array}{l}\text { Heliconia metallica Planch. \& Linden ex } \\
\text { Hook. }\end{array}$ & Swarga pakhi & Gr & Herb, er; pl & Exotic $M A R 2708$ & $\mathrm{O}$ \\
\hline H. psittacorum L. f. & Tiathuti & $\mathrm{Gr}$ & Herb, er; $\mathrm{pl}$ & Exotic $M A R 2709$ & $\mathrm{O}$ \\
\hline H. rostrata Ruiz \& Pav. & Chingrinomi & Gr & Herb, er; pl & Exotic MAR 2800 & $\mathrm{O}$ \\
\hline \multicolumn{6}{|l|}{ Musaceae Juss. } \\
\hline \multicolumn{6}{|l|}{ Zingiberaceae Martinov } \\
\hline Alpinia calcarata (Haw.) Roscoe & Deshi choto elachi & $\mathrm{Gr}$ & Herb, er; cv & Exotic SSS 2151 & $\mathrm{M}, \mathrm{Sp}$ \\
\hline A. conchigera Griff. & Konchi elachi & Gr & Herb, er; cv & Native SSS 2152 & M \\
\hline Curcuma longa $\mathrm{L}$. & Halud & $\mathrm{Fl}, \mathrm{Hs}$ & Herb, er; cv & Exotic SSS 2153 & $\mathrm{Sp}$ \\
\hline C. zedoaria (Christm.) Rosc. & Sathi & $\mathrm{Fl}, \mathrm{Rs}, \mathrm{Sj}, \mathrm{Wl}$ & Herb, er; w & Native SS 509 & M \\
\hline Elettaria cardamomит (L.) Maton & Soto elachi & $\mathrm{Gr}, \mathrm{Hs}$ & Herb, er; pl & Exotic SSS 2154 & $\mathrm{Sp}$ \\
\hline Hedychium coronarium J. König & Dolon chapa & Gr, Hs, Rs & Herb, er; cv & Exotic SSS 2155 & $\mathrm{O}$ \\
\hline Zingiber officinale Roscoe & Ada & $\mathrm{Fl}, \mathrm{Hs}$ & Herb, er; cv & Exotic SSS 2156 & Sp \\
\hline Z. zerumbet (L.) Roscoe ex Sm. & Mohaboribotch & $\mathrm{Fl}, \mathrm{Hs}, \mathrm{Sj}$ & Herb, er; w & Native SSS 2157 & M \\
\hline \multicolumn{6}{|l|}{ Costaceae Nakai } \\
\hline $\begin{array}{l}\text { Cheilocostus speciosus (J. Koenig) C.D. } \\
\text { Specht }\end{array}$ & Keomul & $\mathrm{Bw}^{2}, \mathrm{Fl}, \mathrm{Lb}, \mathrm{Rs}$ & Herb, er; w & Native SS 296 & M \\
\hline Costus woodsonii Maas & Lipistic plant & Gr & Herb, er; cv & Exotic MAR 2801 & $\mathrm{O}$ \\
\hline \multicolumn{6}{|l|}{ Cannaceae Juss. } \\
\hline Canna indica $\mathrm{L}$. & Kolabati & Gr, Hs, Rs & Herb, er; pl-w & Exotic SSS 2158 & $\mathrm{M}, \mathrm{O}$ \\
\hline \multicolumn{6}{|l|}{ Marantaceae R. Br. } \\
\hline $\begin{array}{l}\text { Schumannianthus dichotomus (Roxb.) } \\
\text { Gagnep. }\end{array}$ & Pati-pata & Hs, Wtl & Shrub; cv-w & Native $G M H 5126$ & $\mathrm{Hc}$ \\
\hline \multicolumn{6}{|l|}{ Pontederiaceae Kunth } \\
\hline Eichhornia crassipes (Mart.) Solms & Kachuripana & Wtl & Herb, ff; w & Exotic SS 345 & Fo \\
\hline Monochoria hastata (L.) Solms & Bara nukha & Wtl & Herb, em; w & Native $S S 347$ & M \\
\hline M. vaginalis (Burm. f.) C. Presl & Nukha & Wtl & Herb, em; w & Native MAR 159 & M \\
\hline
\end{tabular}


Table 1 contd.

\begin{tabular}{|c|c|c|c|c|c|}
\hline Scientific name & Bangla name & Habitat & Habit & Origin RSE & Use \\
\hline \multicolumn{6}{|l|}{$\begin{array}{l}\text { Amaryllidaceae J. St.-Hil. } \\
\text {. }\end{array}$} \\
\hline Allium cepa $\mathrm{L}$. & Piyaj & Hs & Herb, er; cv & Exotic $M A R 2807$ & $\mathrm{Sp}$ \\
\hline A. sativum $\mathrm{L}$. & Rashun & Hs & Herb, er; cv & Exotic MAR 2808 & $\mathrm{Sp}$ \\
\hline Crinum asiaticum $\mathrm{L}$. & Shukdarshan & $\mathrm{Gr}, \mathrm{Hs}$ & Herb, er; pl & Native $S S 411$ & $\mathrm{M}, \mathrm{O}$ \\
\hline C. latifolium $\mathrm{L}$. & \multicolumn{2}{|c|}{ Baro-Shukdarshan Gr, Hs } & Herb, er; pl & Native SSS 2161 & $\mathrm{O}$ \\
\hline Scadoxus multiflorus (Martyn) Raf. & Agnigolock & $\mathrm{Gr}, \mathrm{Hs}$ & Herb, er; cv & Exotic SSS 2162 & $\mathrm{M}, \mathrm{O}$ \\
\hline $\begin{array}{l}\text { Urceolina } \times \text { grandiflora } \text { (Planch. } \& \\
\text { Linden) Traub }\end{array}$ & Eucharis lily & $\mathrm{Gr}$ & Herb, er; pl & Exotic MAR 2729 & $\mathrm{O}$ \\
\hline $\begin{array}{l}\text { Zephyranthes minuta (Kunth) D. Dietr. } \\
\text { Asparagaceae Juss. }\end{array}$ & \multicolumn{2}{|c|}{ Golapi ghash phul Gr, Hs } & Herb, er; pl & Exotic SSS 2163 & $\mathrm{O}$ \\
\hline Agave americana $\mathrm{L}$. & Shatabdi udvid & $\mathrm{Gr}, \mathrm{Hs}$ & Herb, er; cv & Exotic $M A R 2802$ & $\mathrm{O}$ \\
\hline A. vivipara $\mathrm{L}$. & Unknown & $\mathrm{Gr}, \mathrm{Hs}$ & Herb, er; cv & Exotic MAR 2803 & $\mathrm{O}$ \\
\hline Asparagus racemosus Wild. & Shatamuli & $\mathrm{Gr}, \mathrm{Hs}, \mathrm{Sj}$ & Herb, vi; w & Native SS 410 & M \\
\hline Cordyline fruticosa (L.) A. Chev. & Agnishwar & $\mathrm{Gr}, \mathrm{Hs}$ & Herb, er; cv & Exotic SSS 2159 & M \\
\hline Dracaena fragrans (L.) Ker Gawl. & Gondhi drakan & $\mathrm{Gr}, \mathrm{Hs}$ & Shrub; pl & Exotic MAR 2804 & $\mathrm{O}$ \\
\hline D. spicata Roxb. & Kado drakan & $\mathrm{Gr}, \mathrm{Hs}$ & Shrub; pl & Native $M A R 2805$ & $\mathrm{O}$ \\
\hline Furcraea foetida (L.) Haw. & Gandhohemp & $\mathrm{Gr}, \mathrm{Hs}$ & Shrub; pl & Exotic MAR 2806 & $\mathrm{O}$ \\
\hline Sansevieria trifasciata Prain & Sutahara & $\mathrm{Gr}, \mathrm{Hs}$ & Herb, er; cv & Exotic SSS 2160 & $\mathrm{O}$ \\
\hline \multicolumn{6}{|l|}{ Colchicaceae DC. } \\
\hline Gloriosa superba L. & Ulatchandal & Gr, Hs, Wl & Herb, vi; pl-w & Native SS 510 & M \\
\hline \multicolumn{6}{|l|}{ Hypoxidaceae R. Br. } \\
\hline Curculigo orchioides Gaertn. & Talmuli & $\mathrm{Sj}, \mathrm{Wl}$ & Herb, er; w & Native $M A R 164$ & M \\
\hline \multicolumn{6}{|l|}{ Xanthorrhoeaceae Dumort. } \\
\hline Aloe vera (L.) Burm. f. & Ghritakumari & $\mathrm{Gr}, \mathrm{Hs}$ & Herb, er; cv-w & Exotic SSS 2164 & $\mathrm{M}, \mathrm{O}$ \\
\hline \multicolumn{6}{|l|}{ Smilacaceae Vent. } \\
\hline Smilax ovalifolia Roxb. & Kumarika & $\mathrm{Sj}, \mathrm{Wl}$ & Herb, vi; w & Native $S S 313$ & M \\
\hline S. perfoliata Lour. & Kumari lata & $\mathrm{Sj}, \mathrm{Wl}$ & Herb, vi; w & Native $S S 511$ & M \\
\hline \multicolumn{6}{|l|}{ Dioscoreaceae R. Br. } \\
\hline Dioscorea alata $\mathrm{L}$. & Chupri alu & $\mathrm{Hs}, \mathrm{Sj}$ & Herb, tw; cv-w & Native SSS 2167 & $\mathrm{Vg}$ \\
\hline D. belophylla (Prain) Voigt ex Haines & Shora alu & $\mathrm{Hs}, \mathrm{Lb}, \mathrm{Wl}$ & Herb, tw; cv-w & Native $M A R 175$ & M \\
\hline D. bulbifera $\mathrm{L}$. & Ban alu & $\mathrm{Hs}, \mathrm{Lb}, \mathrm{Sj}$ & Herb, tw; cv-w & Native $M A R 2810$ & M \\
\hline D. esculenta (Lour.) Burkill & Mou alu & $\mathrm{Gr}, \mathrm{Sj}, \mathrm{Wl}$ & Herb, tw; cv-w & Native $M A R 2811$ & $\mathrm{Vg}$ \\
\hline D. hamiltonii Hook. f. & Dud alu & $\mathrm{Gr}, \mathrm{Sj}, \mathrm{Wl}$ & Herb, tw; cv-w & Native $S S 129$ & $\mathrm{M}, \mathrm{Vg}$ \\
\hline D. pentaphylla $\mathrm{L}$. & Jhum alu & $\mathrm{Gr}, \mathrm{Sj}, \mathrm{Wl}$ & Herb, tw; cv-w & Native SSS 2168 & $\mathrm{M}, \mathrm{Vg}$ \\
\hline \multicolumn{6}{|l|}{ Orchidaceae Juss. } \\
\hline Acampe ochracea (Lindl.) Hochr. ++ & Kampera & $\mathrm{Gr}$ & Herb, er; w & Native $G M H 5127$ & $\mathrm{O}$ \\
\hline A. praemorsa (Roxb.) Blatt. \& McCann & Mar & $\mathrm{Gr}$ & Herb, er; w & Native $G M H 5128$ & $\mathrm{O}$ \\
\hline Bulbophyllum lilacinum Ridl. ++ & Gota parchallow & $\mathrm{Gr}$ & Herb, ep; w & Native $G M H 5129$ & $\mathrm{O}$ \\
\hline B. roxburghii (Lindl.) Rchb.f. & Unknown & $\mathrm{Gr}$ & Herb, ep; w & Native $G M H 5130$ & $\mathrm{O}$ \\
\hline Cymbidium aloifolium (L.) Sw. \# & Tosabak & $\mathrm{Gr}$ & Herb, ep; w & Native $S S 512$ & $\mathrm{M}, \mathrm{O}$ \\
\hline Dendrobium anceps $\mathrm{Sw}$. & Asiriam & Gr & Herb, ep; w & Native GMH 5131 & $\mathrm{M}, \mathrm{O}$ \\
\hline D. aphyllum (Roxb.) C.E.C. Fisch. & Fasiariam & $\mathrm{Gr}$ & Herb, ep; w & Native $G M H 5132$ & $\mathrm{M}, \mathrm{O}$ \\
\hline Geodorum densiflorum (Lam.) Schltr. & Sankhamul & $\mathrm{Sj}, \mathrm{Wl}$ & Herb, er; w & Native $M A R 133$ & $\mathrm{O}$ \\
\hline Habenaria diphylla Dalzell & Bhumi orchid & $\mathrm{Gl}, \mathrm{Lb}, \mathrm{Wl}$ & Herb, er; w & Native $M A R 185$ & $\mathrm{O}$ \\
\hline Luisia brachystachys (Lindl.) Blume & Luisia & $\mathrm{Gr}$ & Herb, ep; w & Native GMH 5133 & $\mathrm{O}$ \\
\hline Nervilia gammieana (Hook. f.) Pfitzer & Unknown & W1 & Herb, er; w & Exotic $M A R 176$ & $\mathrm{O}$ \\
\hline
\end{tabular}


Table 1 contd.

\begin{tabular}{|c|c|c|c|c|c|}
\hline Scientific name & Bangla name & Habitat & Habit & Origin RSE & Use \\
\hline Oberonia gammiei King \& Pantl. ++ & Unknown & $\mathrm{Gr}$ & Herb, ep; w & Native GMH 5134 & $\mathrm{O}$ \\
\hline Papilionanthe teres (Roxb.) Schltr. & Paphoteri & $\mathrm{Gr}$ & Herb, ep; pl & Native $G M H 5135$ & $\mathrm{O}$ \\
\hline Rhynchostylis retusa (L.) Blume & Kopou phool & $\mathrm{Gr}$ & Herb, ep; pl & Native GMH 5136 & $\mathrm{M}, \mathrm{O}$ \\
\hline $\begin{array}{l}\text { Vanda tessellata (Roxb.) Hook. ex G. } \\
\text { Don. }\end{array}$ & Rasna & Op & Herb, ep; w & Native $G M H 5138$ & M \\
\hline $\begin{array}{l}\text { Zeuxine nervosa (Wall. ex Lindl.) Benth. } \\
\text { ex Trimen }++\end{array}$ & Nervoxine orchid & $\mathrm{Fl}, \mathrm{Wl}$ & Herb, er; w & Native GMH 5139 & M \\
\hline
\end{tabular}

Notes: Habitat: Fl- Fallow land, Hs- Homestead, Gl- Grassland, Gr- Garden, Ml- Marginal land, Lb- Lake bank, Bw ${ }^{1}-$ On brick wall (with complete life cycle), $\mathrm{Bw}^{2}$ - Op- On plant (only seedling or sapling stage), Rs- Roadside, $\mathrm{Sj}$ - Scrub jungle, Wl- Woodland, Wtl- Wetland; Habit: cr- creeper, cv- cultivated, em- emergent, er-erect, ff- free floating, fr- floating with rooted, sm- submerged, pl- planted pr-prostrate, ps-parasite, vi-vine, l-large, m-medium, s-small, sc-scandant, w- wild; Use: Ap- aquarium plant, Bc-Bamboo crafts, Bv- Beverage, Cc- Cane crafts, Co- Cosmetics, Dy- Dye yielding, Ed- Edible, Fb- Fibre, Fd- Fodder, Ff- Fish feed, Fn- Fence, Fo- Forage, Fr- Fruit, Fu- Fuel, Fw- Fuel wood, Gm- Green manure, GuGum, Hc- Handicrafts, Ju- Juice, M- Medicine, Mu- Mulching, O- Ornamental, Oy- Oil yielding, Nk- Not known, PcPickle, Pf- Perfume, Pp- Paper pulp, Po- Poisionous, Pu- Pulse, Sb- Soil binder, Sd- Shade providing, Sf- Silkworm feed, Sp- Spice, T- Timber, Tn- Tannin, Vg- Vegetable, Wp- Water purify, Wd- Weed; *- Artificial/naturalized hybrid; \#Reported as threatened in Bangladesh; +- Exotic species introduced in the study area in recent years; ++- Native species settled in the study area in recent years. RSE: GMH- Gazi Mosharof Hossain, MAR- Md. Abdur Rahim, SAK- Saleh Ahammad Khan, SS- Sharmin Sultana, SSS- Shayla Sharmin Shetu.

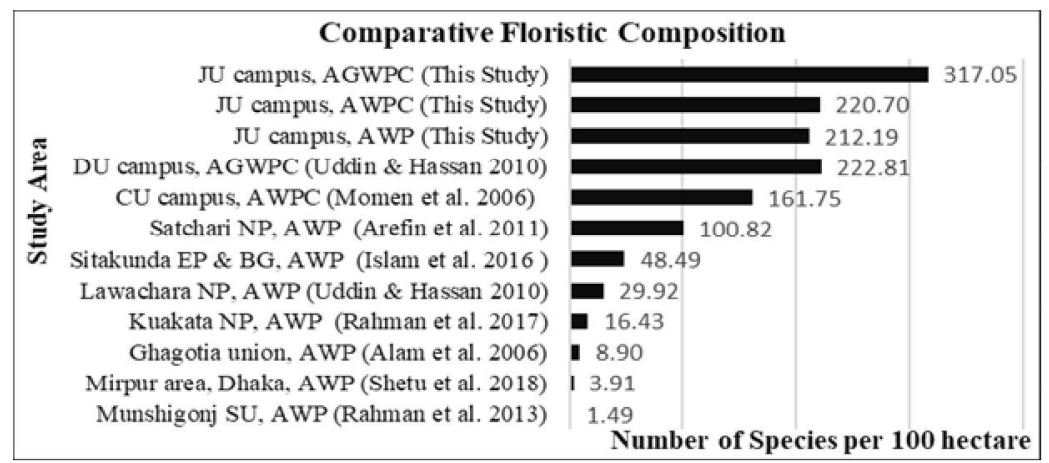

Fig. 4. Floristic composition in selected campus (DU, CU), (semi-)urban (SU), urban (Mirpur), rural (Ghagotia) and forest (EP, NP) areas of Bangladesh (CU- University of Chittagong; DU- University of Dhaka; JU- Jahangirnagar University; BG- Botanical Garden, EC- Ecopark, NP- National park; SUSadar upazila; A- Angiosperms; C- Cultivated; G- Gymnosperms; P- Planted; W- Wild).

Traditionally, the management of this campus is authoritative, visionary and democratic. The anthropogenic activities throughout the area are monitored and controlled through respective offices, departments and committees under the university authority. In this campus, one Botanical Garden, one Plant Conservatory and one Wildlife Rescue Centre (WRC) housing wild, cultivated and planted species have been established. A number of gardens (currently 20) are regularly set up throughout the study area that harbour mostly ornamental and medicinal or different weed species. Some open parts of the campus, composed of fallow lands, wetlands, scrub jungles or forest patches and covered with dense vegetation (Fig. 1), have been maintained as undemarcated infrastructure or settlement free area since many years. These open parts have recently been demarcated in the master plan of the campus by the administration to maintain and improve their natural features and the bio-ecological resources. Besides, a strong public awareness for conservation of biodiversity of the area is prevailing among the inhabitants. 
In course of time, the original vegetation cover of this area has been changed in different magnitudes. However, based on last three decade's field observation and the comparative images on this area collected from Google Earth Pro, its present status is inferred to be richer in species composition and density in respect to its previous state (Fig. 1). It might be due to natural and artificial land transformation, increase in soil fertility, introduction of diaspores of different plant species from different regions of the country through various biotic and abiotic agents, successful adaptation of many exotic species with its diverse ecosystems, effective management of the whole campus including its open infra-structure free semi-natural areas, gardens, conservatory, WRC etc. and existing strong public awareness among the inhabitants for conservation of its biodiversity. The vegetation of this area provides the habitats for various animal groups (mammals, birds, reptiles, molluscs, amphibians, fishes, and insects etc.) including the migratory birds and supports the feeding, nesting sites and breeding ground for many of them (Mahony et al., 2009; Jahan et al., 2018).

In some parts of the study area, different anthropogenic activities including firing, crop and fish cultivation, grazing, leaf litter and fuel wood collection, and huge visits etc. are occasionally and irregularly functional as the major threats to its biodiversity. However, this area is still harbouring a rich biodiversity with a huge number of plant species in its diverse ecosystems and habitats including the 15 plant species threatened in Bangladesh due to which it can be considered as an exceptional semi-natural campus of a semi-urban area that can serve as an excellent center of in situ and ex situ biodiversity conservation. To achieve this goal, strengthening and improving the existing management of the campus area with adopting necessary conservation initiatives including policies, legislation, controlling and minimizing anthropogenic interferences, increasing the public awareness, improving the quality of major natural habitats and construction of the remaining boundary wall around the area etc. are highly recommended. Adequate protection, conservation and development of natural resources of this area will improve its sustainable socioeconomic and ecological services besides its mainstream institutional contributions.

\section{Acknowledgements}

The authors are grateful to the Authority and Department of Botany of Jahangirnagar University and Bangladesh National Herbarium for their cooperation in this study and the people who were directly or indirectly involved in conducting and publishing this study.

\section{References}

Ahmed, Z.U., Begum, Z.N.T., Hassan, M.A., Khondker, M., Kabir, S.M.H., Ahmad, M., Ahmed, A.T.A., Rahman, A.K.A. and Haque, E.U. (Eds). 2008-2009. Encyclopedia of Flora and Fauna of Bangladesh. Vols. 6-8 \& 12. Asiatic Society of Bangladesh, Dhaka.

Ahmed, Z.U., Hassan, M.A., Begum, Z.N.T., Khondker, M., Kabir, S.M.H., Ahmad, M., Ahmed, A.T.A. (Eds). 2009. Encyclopedia of Flora and Fauna of Bangladesh. Vols. 9-10. Asiatic Society of Bangladesh, Dhaka.

Alam, M.S., Hassan, M.A. and Uddin, M.Z. 2006. A Preliminary Checklist of the angiospermic flora of Ghagotia union under Kapasia Upazila in Gazipur District, Bangladesh. Bangladesh J. Plant Taxon. 13(2): 155-170.

Ara, H., Khan, B. and Uddin, S.N. (Eds). 2013. Red Data Book of Vascular Plants of Bangladesh. Vol. 2. Bangladesh National Herbarium, Dhaka, pp. 1-280.

Arefin, M., Rahman, M., Uddin, M. and Hassan, M. 2011. Angiosperm flora of Satchari National Park, Habiganj, Bangladesh. Bangladesh J. Plant Taxon. 18(2): 117-140. 
Cazaux, G., Carels, K. and Van Gijseghem, D. 2007. Prospects and challenges for agricultural diversification in a peri-urban region (Flanders-Belgium). Beleidsdomein Landbouw en Visserij, Afdeling Monito-ring en Studie, Brussel, pp. 1-29.

Cronquist, A. 1981. An integrated system of classification of flowering plants. Columbia University Press, New York, $1262 \mathrm{pp}$.

Farhana, H., Hossain, A.B.M.E., Khan, S.A. and Islam, M.A. 1997. Ecological and distributional studies on the pteridophytic flora of Jahangirnagar University campus. Bangladesh J. Plant Taxon. 4(1): 67-75.

Freitas, E.V.D., Veloso, M.D.M. and Araújo, W.S. 2020. Urbanization alters the composition, but not the diversity and structure of Neotropical savanna woody plant communities. Folia Geobotanica 55: 95108.

Ghani, A. 2003. Medicinal Plants of Bangladesh with Chemical Constituents and Uses. Asiatic Society of Bangladesh, Dhaka, Bangladesh.

Goddard, M.A., Dougill, A.J. and Benton, T.G. 2009. 'Scaling up from gardens: Biodiversity conservation in urban environments'. Trends in Ecology and Evolution 25: 90-98.

Haque, A.K.M.K., Khan, S.A., Uddin, S.N. and Shetu, S.S. 2018. An annotated checklist of the Angiospermic flora of Rajkandi Reserve Forest of Moulvibazar, Bangladesh. Bangladesh J. Plant Taxon. 25(2): 187-207.

Hooker, J.D. 1872-1897. The Flora of British India. Vols. 1-7. L. Reeve \& Co., Ashford, Kent.

Hossain, A.B.M.E., Alam, M.D., Rahim, M.A. and Rahim, M.A. 2009. A Pictorial Guide to Nursery Plants. Jahangirnagar University, Savar, Dhaka-1342, pp. 1-247.

Hossain, A.B.M.E., Khan, S.A. and Islam, M.A. 1995. An inventory of plant diversity in relation with the ecology and environment of Jahangirnagar University. Vegetational composition and their taxonomic identity. Bangladesh J. Life Sci. 7(1\&2): 95-103.

Hossain, M.K. and Pasha, M.K. 2001. Alien Invasive Plants in Bangladesh and their Impacts on the Ecosystem. In: Assessment and Management of Alien Species that Threatened Ecosystem, Habitats and Species. CBD Technical Paper No. 21. Secretariat of the Convention on Biological Diversity. Montreal, Canada, pp.73-75.

Hunter, P. 2007. 'The human impact on biological diversity'. EMBO Reports 8: 316-318.

Huq, A.M. 2019. Plant names of Bangladesh. Ashrafia Boi Ghor, 36, Banglabazar, Dhaka, pp. 1-578.

Islam, M.A., Rahman, M.M. and Hossain, G.M. 2016. Floristic composition and phytodiversity status of Sitakunda Ecopark, Chittagong, Bangladesh. Jahangirnagar University J. Biol. Sci. 5(1): 29-45.

Jahan, I., Begum, S., Feeroz, M.M., Das, D.K. and Datta, A.K. 2018. Nesting pattern of birds in Jahangirnagar University Campus, Bangladesh. Journal of Threatened Taxa 10(5): 11618-11635.

Khan, M.S. and Huq, A.M. 2001. The vascular flora of Chunati wildlife sanctuary in south Chittagong. Bangladesh J. Plant Taxon. 8(1): 47-64.

Khan, M.S., Rahman, M.M. and Ali, M.A. (Eds). 2001. Red Data Book of Vascular Plants of Bangladesh. Vol. 1. Bangladesh National Herbarium, Dhaka, Bangladesh, pp. 1-179.

Khanam, R., Khan, S.A. and Rahim, M.A. 2020. Angiosperms in Narsingdi District of Bangladesh: Class Magnoliopsida. Bangladesh J. Plant Taxon. 27(1): 153-171.

Kowarik, I. 2011. Novel urban ecosystems, biodiversity, and conservation. Environ Pollut 159: 1974-1983.

Kramer, K.U. and Green, P.S. 1990. Pteridophytes and Gymnosperms. In: Kubitzki, K. The families and genera of vascular plants. Vol. 1. Springer, Berlin.

Mahony, S., Hasan, M.K., Kabir, M.M., Ahmed, M. and Hossain, M.K. 2009. A catalogue of amphibians and reptiles in the collection of Jahangirnagar University, Dhaka, Bangladesh, Hamadryad, 34(1): 80-94.

McKinney, M.L. 2006. Urbanization as a major cause of biotic homogenization. Biological Conservation 127: 247-60.

Meeus, S.J. and Gulinck, H. 2008. Semi-Urban Areas in Landscape Research: A Review. Living Rev. Landscape Res. 2: 1-30. 
Momen, R.U., Hossain, M.K., Huda, S.M.S. and Khan, B.M. 2006. Assessment of plant diversity in Chittagong University campus. J. of Forestry \& Env. 4: 27-52.

Pasha, M.K. and Uddin, S.B. 2013. Dictionary of Plant Names of Bangladesh (Vascular Plants). Janokalyan Prokashani, Andarkilla, Chittagong, pp. 1-320.

Pichi, S.R.E.G. 1977. Tentamen pteridophytorum genera in taxonomicum ordinem redigendi. Webbia 31: 313-512.

POWO, 2020. Plants of the World Online. Facilitated by the Royal Botanic Gardens, Kew. Published on the Internet; http://www.plantsoftheworldonline.org/. Retrieved on 21 December 2020.

Prain, D. 1903. Bengal Plants. Vol. 2. Indian Reprint 1963. Botanical Survey of India, Calcutta, pp. 491-1013.

Rahaman, M.A., Rahman, M.A. and Uddin, M.Z. 2017. Diversity of angiosperm flora of Kuakata National Park, Patuakhali District. Bangladesh. J. Asiat. Soc. Bangladesh, Sci. 43(2): 143-159.

Rahman, M.A. 2017. Plant Diversity in Hazarikhil Wildlife Sanctuary of Chittagong and its Conservation Management. J. biodivers. Conserv. Bioresour. Manag. 3(2): 43-56.

Rahman, M.O., Begum, M. and Ullah, M.W. 2013. Angiosperm Flora of Sadar Upazila of Munshiganj District, Bangladesh. Bangladesh J. Plant Taxon. 20(2): 213-231.

Rahman, M.S., Hossain, M.G., Khan, S.A. and Uddin, S.N. 2015. An Annotated Checklist of the Vascular Plants of Sundarban Mangrove forest of Bangladesh. Bangladesh J. Plant Taxon. 22(1): 17-41.

Roy, G.K. and Khan, S.A. 2020. Preliminary Taxonomic Study on Homestead Flora of Four Districts of Bangladesh: Magnoliopsida. Bangladesh J. Plant Taxon. 27(1): 37-65.

Schaminée, J.H.J., Janssen, J.A.M., Hennekens, S.M. and Ozinga, W.A. 2011. Large vegetation databases and information systems: new instruments for ecological research, nature conservation, and policy making. Plant Biosyst 145: 85-90.

Sharrock, S. 2012. Global Strategy for Plant Conservation: A guide to the GSPC. All the targets, objectives and facts. Botanic Gardens Conservation International (2012). Richmond, UK.

Shetu, S.S., Khan, S.A. and Uddin, S.N. 2018. Checklist of Angiosperms extant in Mirpur area of Dhaka City. Jahangirnagar University J. Biol. Sci. 7(2): 47-64.

Siddiqui, K.U., Islam, M.A., Ahmed, Z.U., Begum, Z.N.T., Hassan, M.A., Khondker M., Rahman, M.M., Kabir, S.M.H., Ahmed, M., Ahmed, A.T.A., Rahman, A.K.T. \& Haque, E.U. (Eds). 2007. Encyclopedia of Flora and Fauna of Bangladesh. Vols. 5 \& 11. Asiatic Society of Bangladesh, Dhaka.

Sultana, M. 2012. Taxonomic and ethnobatanical studies on the angiospermic flora of Patuakhali District in Bangladesh. Ph.D. Thesis (Unpublished). Department of Botany, University of Dhaka.

Tabassum, R. 2015. Angiospermic flora of Gazipur District, Bangladesh. Ph.D. Thesis (Unpublished). Department of Botany, University of Dhaka, pp. 1-707.

The Plant List, 2013. The Plant List, a working list of all plant species. Version $1.1<\mathrm{http}: / / \mathrm{www}$. theplantlist.org/>. Accessed on 15 April, 2021.

TROPICOS, 2021. Tropicos.org. Missouri Botanical Garden. http://www.tropicos.org, Missouri Botanical Garden-4344, Shaw Boulevard - Saint Louis, Missouri 63110. Accessed on 25 March 2021.

Uddin, M.Z. and Hassan, M.A. 2004. Flora of Rema-Kalenga Wildlife Sanctuary. IUCN Bangladesh Country Office, Dhaka, Bangladesh, pp. 1-120.

Uddin, M.Z. and Hassan, M.A. 2010. Angiosperm diversity of Lawachara National Park (Bangladesh): a preliminary assessment. Bangladesh. J. Plant Taxon. 17(1): 9-22.

Uddin, M.Z. and Hassan, M.A. 2016. Plant Diversity of Dhaka University campus, Bangladesh. J. Asiat. Soc. Bangladesh, Sci. 42(1): 49-68.

Wu, Z.Y. and Raven, P.H. (Eds). 1994-2001. Flora of China. Vols. 8, 15-18 and 24. Missouri Botanical Garden Press, St. Louis, USA.

Wu, Z.Y., Raven, P.H. and Hong, D.Y. (Eds). 1999-2013. Flora of China. Vols. 2-7, 9-14, 19-23 and 25. Missouri Botanical Garden Press, St. Louis.

(Manuscript received on 05 June, 2020; revised on 21 February, 2021) 